\title{
SUMMARY OF PUBLISHED AQUATIC TOXICITY INFORMATION AND WATER- QUALITY CRITERIA FOR SELECTED VOLATILE ORGANIC COMPOUNDS
}

U.S. GEOLOGICAL SURVEY

Open-File Report 97-563

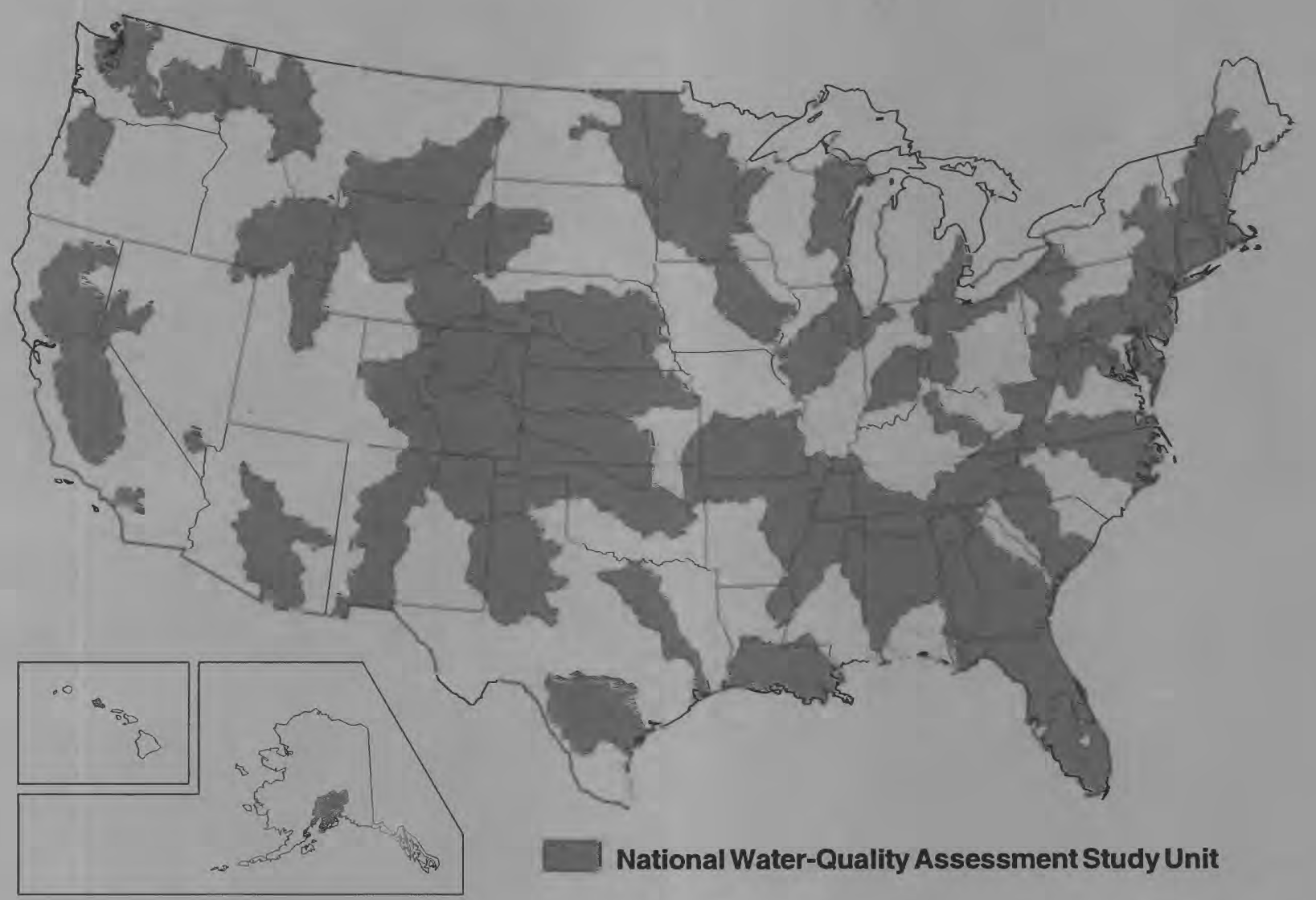

NATIONAL WATER-QUALITY ASSESSMENT PROGRAM

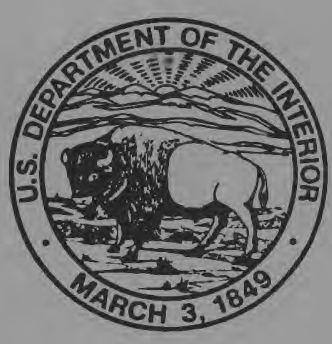




\section{SUMMARY OF PUBLISHED AQUATIC TOXICITY INFORMATION AND WATER- QUALITY CRITERIA FOR SELECTED VOLATILE ORGANIC COMPOUNDS}

By Barbara L. Rowe, Sondra J. Landrigan, and Thomas J. Lopes

U.S. GEOLOGICAL SURVEY

Open-File Report 97-563

Rapid City, South Dakota 1997

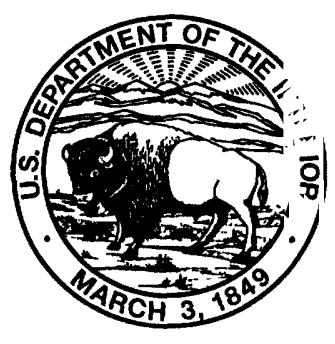




\title{
U.S. DEPARTMENT OF THE INTERIOR \\ BRUCE BABBITT, Secretary
}

\author{
U.S. GEOLOGICAL SURVEY
}

Gordon P. Eaton, Director

The use of firm, trade, and brand names in this report is for identification purposes only and does not constitute endorsement by the U.S. Geological Survey.

For additional information write to:

District Chief, WRD

U.S. Geological Survey

1608 Mt. View Road

Rapid City, SD 57702
Copies of this report can be purchared from:

U.S. Geological Survey

Branch of Information Services

Box 25286

Federal Center

Denver, CO 80225 


\section{FOREWORD}

The mission of the U.S. Geological Survey (USGS) is to assess the quantity and quality of the earth resources of the Nation and to provide information that will assist resource managers and policymakers at Federal, State, and local levels in making sound decisions. Assessment of water-quality conditions and trends is an important part of this overall mission.

One of the greatest challenges faced by waterresources scientists is acquiring reliable information that will guide the use and protection of the Nation's water resources. That challenge is being addressed by Federal, State, interstate, and local water-resource agencies and by many academic institutions. These organizations are collecting water-quality data for a host of purposes that include: compliance with permits and water-supply standards; development of remediation plans for a specific contamination problem; operational decisions on industrial, wastewater, or watersupply facilities; and research on factors that affect water quality. An additional need for water-quality information is to provide a basis on which regional and national-level policy decisions can be based. Wise decisions must be based on sound information. As a society we need to know whether certain types of water-quality problems are isolated or ubiquitous, whether there are significant differences in conditions among regions, whether the conditions are changing over time, and why these conditions change from place to place and over time. The information can be used to help determine the efficacy of existing waterquality policies and to help analysts determine the need for and likely consequences of new policies.

To address these needs, the Congress appropriated funds in 1986 for the USGS to begin a pilot program in seven project areas to develop and refine the National Water-Quality Assessment (NAWQA) Program. In 1991, the USGS began full implementation of the program. The NAWQA Program builds upon an existing base of water-quality studies of the USGS, as well as those of other Federal, State, and local agencies. The objectives of the NAWQA Program are to:

- Describe current water-quality conditions for a large part of the Nation's freshwater streams, rivers, and aquifers.
- Describe how water quality is changing over time.

- Improve understanding of the primary natural and human factors that affect water-quality conditions.

This information will help support the development and evaluation of management, regulatory, and monitoring decisions by other Federal, State, and local agencies to protect, use, and enhance water resources.

The goals of the NAWQA Program are being achieved through ongoing and proposed invest 'gations of 59 of the Nation's most important river basins and aquifer systems, which are referred to as Study Units. These Study Units are distributed throughout the Nation and cover a diversity of hydrogeologic settings. More than two-thirds of the Nation's freshwater use occurs within the 59 Study Units and more than two-thirds of the people served by public watersupply systems live within their boundaries.

National synthesis of data analysis, based on aggregation of comparable information obtained from the Study Units, is a major component of the program. This effort focuses on selected water-quality topics using nationally consistent information. Comparative studies will explain differences and similaritie in observed water-quality conditions among stud ' areas and will identify changes and trends and their causes. The first topics addressed by the national syntresis are pesticides, nutrients, volatile organic compour ds, and aquatic biology. Discussions on these and other waterquality topics will be published in periodic surnmaries of the quality of the Nation's ground and surfare water as the information becomes available.

This report is an element of the comprehensive body of information developed as part of the NAWQA Program. The program depends heavily on the advice, cooperation, and information from many Federal, State, interstate, Tribal, and local agencies and the public. The assistance and suggestions of all are greatly appreciated.

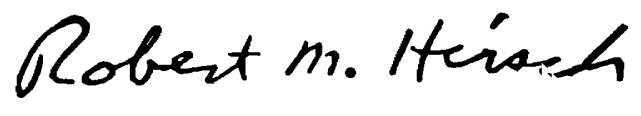

Robert M. Hirsch Chief Hydrolc ơist 


\section{CONTENTS}

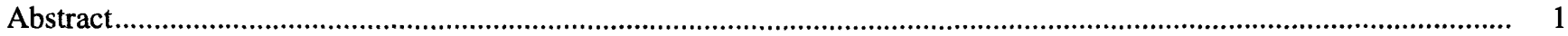

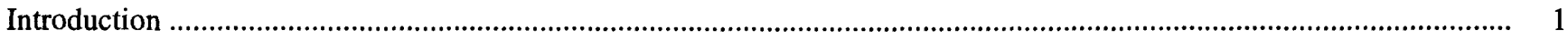

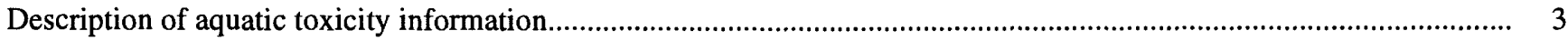

Retrieval from AQUIRE

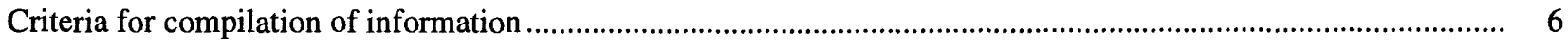

Lowest concentrations of volatile organic compounds that affect a species ...................................................... 6

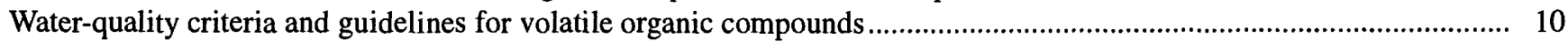

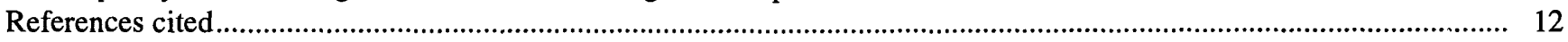

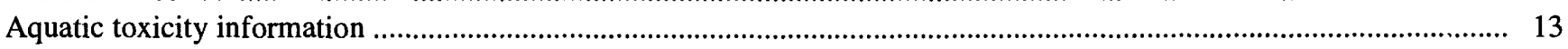

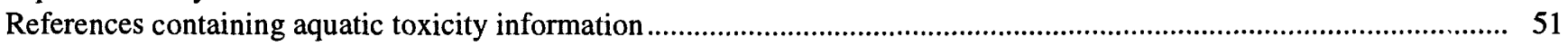

\section{ILLUSTRATION}

1. Map showing location of National Water-Quality Assessment Program Study Units and their proposed implementation dates.

\section{TABLES}

1. Volatile organic compounds measured by the U.S. Geological Survey National Water-Quality Assessment Program

2. Volatile organic compounds with no available aquatic toxicity information

3. Lowest concentrations of volatile organic compounds that affect a species......................................................... 7

4. Water-quality criteria and guidelines for volatile organic compounds .................................................... 11

5. Aquatic toxicity information for selected volatile organic compounds measured by the U.S. Geological Survey

6. References containing aquatic toxicity information for selected volatile organic compounds as described in this study 


\title{
Summary of Published Aquatic Toxicity Information and Water-Quality Criteria for Selected Volatile Organic Compounds
}

\author{
By Barbara L. Rowe, Sondra J. Landrigan, and Thomas J. Lopes
}

\section{ABSTRACT}

Volatile organic compounds (VOCs) are being measured in stream samples as part of the U.S. Geological Survey's National Water-Quality Assessment (NAWQA) Program. Published freshwater aquatic toxicity information and waterquality criteria for VOCs were compiled to compare with the measured concentrations. Aquatic toxicity information for VOCs was retrieved from the U.S. Environmental Protection Agency's (USEPA's) toxicity data base, AQUatic toxicity Information REtrieval (AQUIRE). Toxicity information that had complete or moderately complete documentation was available for 60 of the 87 VOCs that are being measured in water samples. Most toxicity information was from studies of 96 hours or less, and species mortality was typically the endpoint of the toxicity test. A number of published studies using the same species, duration, and endpoint were available for some VOCs. In these instances, only the two studies that reported the lowest concentrations that had an effect were included in the summary. VOCs that had the lowest concentrations that affected a species ranged between $6.5 \mu \mathrm{g} / \mathrm{L}$ (micrograms per liter) for 1,1,2,3,4,4-hexachloro-1,3-butadiene and $5,091,000 \mu \mathrm{g} / \mathrm{L}$ for 2-butanone.

The USEPA and Canadian Council of Resource and Environment Ministers have established water-quality criteria and guidelines for 39 of the 87 VOCs measured in water samples by the NAWQA Program. Criteria and guidelines range from $0.1 \mu \mathrm{g} / \mathrm{L}$ for 1,1,2,3,4,4-hexachloro-1,3butadiene to $860,000 \mu \mathrm{g} / \mathrm{L}$ for 3-chloro-1-propene and chloroethane. These water-quality criteria, guidelines, and toxicity information will be used by NAWQA to assess the status of the Nation's water quality and are available for use by individuals, agencies, and organizations to evaluate the potential effect of VOCs on aquatic life in streams and rivers.

\section{INTRODUCTION}

The National Water-Quality Assessment (NAWQA) Program of the U.S. Geological Survey (USGS) is a systematic assessment of the quality of the Nation's water resources. The primary goals of NAWQA are to describe the status and trends in the quality of a large, representative part of the Nation's surface-water and ground-water resources and to identify the primary natural and human factors affecting the quality of these resources. The design of NAWQA, discussed in detail by Gilliom and others (1995), is organized around 59 Study Units (fig. 1) that include sections of most of the Nation's major river basins and aquifers. The starting dates of Study-Unit Investigations are staggered between fiscal years 1991 and 1997, and at any time water-quality data are being collected in about 16 to 20 Study Units.

Another component of the NAWQA Program is National Synthesis Investigations (NSIs), which are focused on specific groups of contaminants. In part, these investigations compile, analyze, and report water-quality data (collected by Study Units) on a 


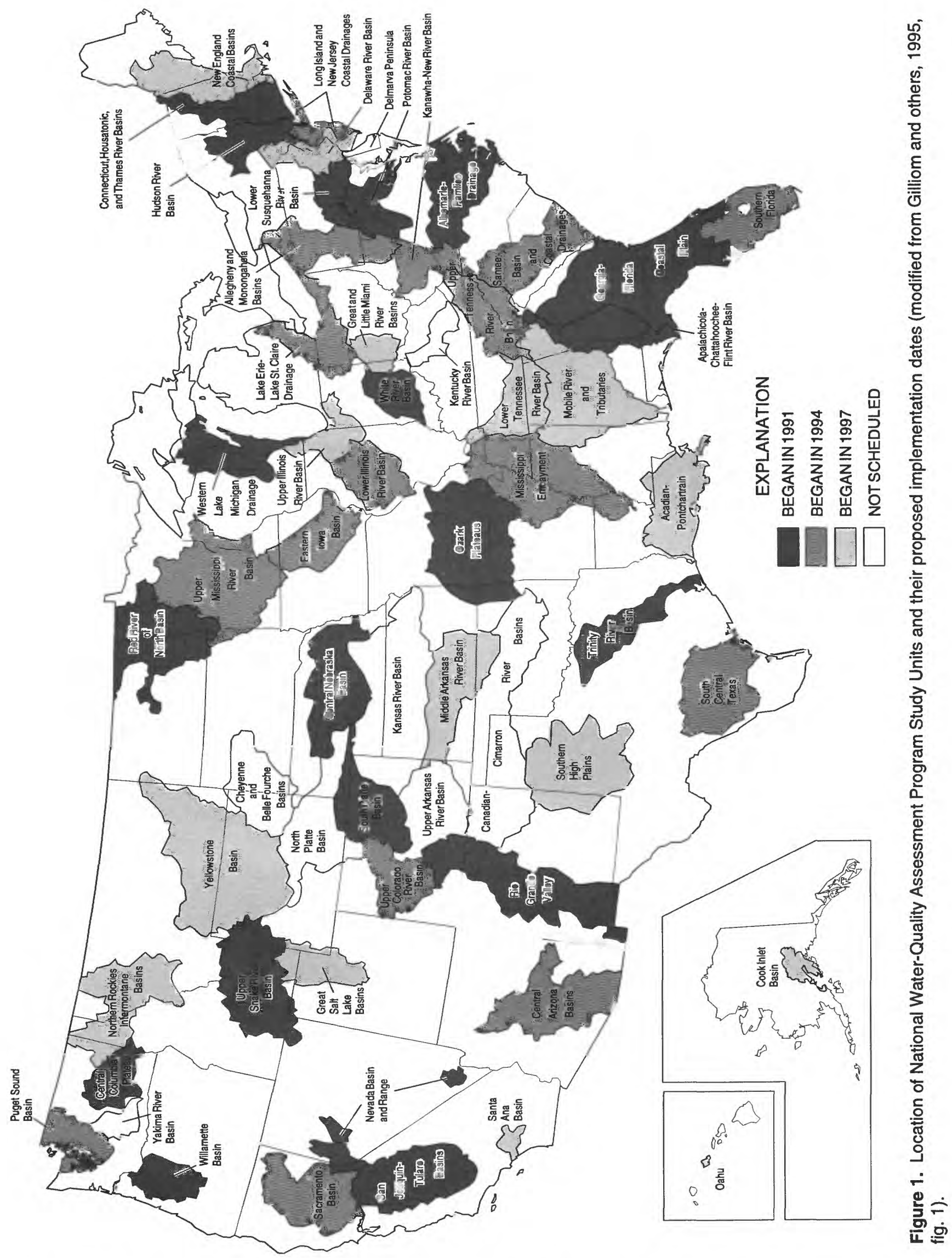


national scale. The NSI for pesticides and the NSI for nutrients began in 1991, and the NSI for volatile organic compounds (VOCs) began in 1993. Two additional NSIs are in the planning process, including aquatic ecology and trace elements. VOCs are being sampled mostly in areas with predominantly residential and commercial land use in metropolitan areas with a population of 250,000 or more (Squillace and Price, 1996; Lopes and Price, 1997).

Water samples collected for the NAWQA Program are analyzed for VOCs at the USGS National Water-Quality Laboratory (NWQL) in Arvada, Colorado, using a new analytical method. This method quantifies low levels of VOCs in ambient surface water and ground water. The method is based upon U.S. Environmental Protection Agency (USEPA) Method 524.2, Revision 4.0 (Eichelberger and Budde, 1989) and USGS Open-File Report 94-708 (Rose and Schroeder, 1994), with improvement of instrumentoperating conditions, increased number of compounds analyzed, modifications to identification criteria, and inclusion of data-reporting strategies for low-level detections. Due to the increasing need for lower concentration data, in part, to identify potentially harmful environmental contaminants in water, the NWQL quantifies 87 analytes at or below the methods detection level if (1) all criteria for analyte identification are met, and (2) the laboratory analysis is not limited by laboratory contamination of VOC samples.

Analytes measured using the new USGS VOC method are divided into two groups: NAWQA target analytes (55 compounds), and other analytes (32 compounds). NAWQA target analytes were selected for emphasis in the NAWQA Program because of their known human-health and (or) aquatic-life concern or because of their high frequency of occurrence in surface water and ground water (John Zogorski, USGS, written commun., 1994). The "other analytes" measured using the new VOC method (table 1) were included because they are on the USEPA's revised method for drinking water.

The purpose of this report is to summarize published aquatic toxicity information and water-quality criteria and guidelines for VOCs that are measured by the NAWQA Program. This published information is needed for comparisons with concentrations measured in streams and rivers by the NAWQA Program and for assessing the status of water quality.

All aquatic toxicity information presented in this report was retrieved from AQUatic toxicity Informa- tion REtrieval (AQUIRE) (U.S. Environmental Protection Agency, 1996a). Toxicity data have been reviewed to update AQUIRE on a quarterly basis during the past 14 years. Data exchanges witl the Organization for Economic Cooperation and Development, Paris, France, and with the Bor k Institute, Nekouz, Yaroslavsky Region, Russian Republic, have made AQUIRE a centralized, international source for toxic effects information.

Water-quality criteria and guidelines were compiled from the Integrated Risk Information System (IRIS) (U.S. Environmental Protection Agency, 1996b) and from the Canadian Council of Resource and Environment Ministers (1991).

\section{DESCRIPTION OF AQUATIC TOXICITY INFORMATION}

Toxicity tests typically are conducted by exposing organisms to a range of contaminant concentrations under controlled conditions and measuring the response of the organisms (Rand and Petrocelli, 1985). Acute toxicity tests are used to determine if solutions are toxic to species during short-duration exposures (typically 96 hours or less), and species mortality is the most common endpoint. Chronic toxicity tests use a longer duration of exposure (typically 7 days o* more). Responses other than species mortality typically are measured during chronic tests and may include growth, reproduction, and behavior. No exact duration or response, however, distinguishes acute and chronic toxicity tests. Laboratory controls are an important part of toxicity tests and are used to ensure that species mortality or other adverse reactions of a test organism are due to the contaminant and not the testing procedure or natural causes. Laboratory controls are performed the same as toxicity tests; however, organisms are not exposed to the contaminant.

The majority of published information for VOCs are median lethal concentration $\left(\mathrm{LC}_{50}\right)$ values, which are the estimated concentrations at which 50 percent of the organisms died during the toxicity test. T'e $\mathrm{LC}_{50}$ is estimated by interpolating species mortality rates from the range in concentrations used in the toxicity test. If the largest concentration did not result in 50-percent mortality, then the $\mathrm{LC}_{50}$ is reporter as greater than the largest concentration. The ef ective mean concentration $\left(\mathrm{EC}_{50}\right)$ is the estimated concentration that affects 50 percent of the organisms; rowever, the endpoint of the test is an effect other than mortality. 
Table 1. Volatile organic compounds measured by the U.S. Geological Survey National Water-Quality Assessment Program [Compounds are identified by the following: PCODE, U.S. Geological Survey parameter code; CAS no., Chemical Abstract Services number; IUPAC, International Union of Pure and Applied Chemistry]

\begin{tabular}{|c|c|c|c|c|c|}
\hline PCODE & CAS no. & $\begin{array}{l}\text { IUPAC compound name } \\
\text { (chemical formula) (common name(s)) }\end{array}$ & PCODE & CAS no. & $\begin{array}{l}\text { IUPAC compound name } \\
\text { (chemical formula) (common name(s)) }\end{array}$ \\
\hline \multicolumn{6}{|c|}{ Target analytes } \\
\hline 34030 & $71-43-2$ & Benzene $\left(\mathrm{C}_{6} \mathrm{H}_{6}\right)$ & 34541 & $78-87-5$ & $\begin{array}{l}\text { 1,2-Dichloropropane }\left(\mathrm{C}_{3} \mathrm{H}_{6} \mathrm{Cl}_{2}\right) \\
\text { (propylene dichloride) }\end{array}$ \\
\hline 32101 & $75-27-4$ & $\begin{array}{l}\text { Bromodichloromethane }\left(\mathrm{CHBrCl}_{2}\right) \\
\text { (dichlorobromomethane) }\end{array}$ & 34704 & \multicolumn{2}{|c|}{$\begin{array}{c}\text { 10061-01-5 cis-1,3-Dichloropropene }\left(\mathrm{C}_{3} \mathrm{H}_{4} \mathrm{Cl}_{2}\right) \\
\text { ((Z)-1,3-dichloropropene) }\end{array}$} \\
\hline 50002 & $593-60-2$ & $\begin{array}{l}\text { Bromoethene }\left(\mathrm{C}_{2} \mathrm{H}_{3} \mathrm{Br}\right) \\
\quad \text { (vinyl bromide) }\end{array}$ & 34699 & \multicolumn{2}{|c|}{$\begin{array}{l}\text { 10061-02-6 trans-1,3-Dichloropropene }\left(\mathrm{C}_{3} \mathrm{H}_{4} \mathrm{Cl}_{2}\right) \\
((\mathrm{E})-1,3 \text {-dichloropropene })\end{array}$} \\
\hline 34413 & 74-83-9 & $\begin{array}{l}\text { Bromomethane }\left(\mathrm{CH}_{3} \mathrm{Br}\right) \\
\text { (methyl bromide) }\end{array}$ & 77135 & $95-47-6$ & $\begin{array}{l}\text { 1,2-Dimethylbenzene }\left(\mathrm{C}_{8} \mathrm{H}_{10}\right) \\
\text { (o-xylene) }\end{array}$ \\
\hline 77342 & $104-51-8$ & $\begin{array}{l}n \text {-Butylbenzene }\left(\mathrm{C}_{10} \mathrm{H}_{14}\right) \\
\text { (1-phenylbutane) }\end{array}$ & 85795 & \multirow{2}{*}{$\begin{array}{l}108-38-3 \\
106-42-3\end{array}$} & \multirow{2}{*}{$\begin{array}{l}\text { 1,3-Dimethylbenzene }\left(\mathrm{C}_{8} \mathrm{H}_{10}\right) \\
\quad(m \text {-xylene) and } \\
\text { 1,4-Dimethylbenzene }\left(\mathrm{C}_{8} \mathrm{H}_{10}\right) \\
(p \text {-xylene })\end{array}$} \\
\hline 34301 & $108-90-7$ & $\begin{array}{l}\text { Chlorobenzene }\left(\mathrm{C}_{6} \mathrm{H}_{5} \mathrm{Cl}\right) \\
\text { (monochlorobenzene) }\end{array}$ & & & \\
\hline 34311 & $75-00-3$ & $\begin{array}{l}\text { Chloroethane }\left(\mathrm{C}_{2} \mathrm{H}_{5} \mathrm{Cl}\right) \\
\text { (ethyl chloride) }\end{array}$ & 77128 & $100-42-5$ & $\begin{array}{l}\text { Ethenylbenzene }\left(\mathrm{C}_{8} \mathrm{H}_{8}\right) \\
\text { (styrene) }\end{array}$ \\
\hline 39175 & $75-01-4$ & $\begin{array}{l}\text { Chloroethene }\left(\mathrm{C}_{2} \mathrm{H}_{3} \mathrm{Cl} \text { ) }\right. \\
\text { (vinyl chloride) }\end{array}$ & 50004 & $637-92-3$ & $\begin{array}{l}\text { 2-Ethoxy-2-methylpropane }\left(\mathrm{C}_{6} \mathrm{H}_{14} \mathrm{O}\right) \\
\text { (ethyl tert-butyl ether, ETBE) }\end{array}$ \\
\hline 34418 & $74-87-3$ & $\begin{array}{l}\text { Chloromethane }\left(\mathrm{CH}_{3} \mathrm{Cl}\right) \\
\text { (methyl chloride) }\end{array}$ & 34371 & $100-41-4$ & $\begin{array}{l}\text { Ethylbenzene }\left(\mathrm{C}_{8} \mathrm{H}_{10}\right) \\
\quad \text { (phenylethane) }\end{array}$ \\
\hline 82625 & $96-12-8$ & $\begin{array}{l}\text { 1,2-Dibromo-3-chloropropane }\left(\mathrm{C}_{3} \mathrm{H}_{5} \mathrm{Br}_{2} \mathrm{Cl}\right) \\
\text { (dibromochloropropane, DBCP) }\end{array}$ & 39702 & $87-68-3$ & $\begin{array}{l}\text { 1,1,2,3,4,4-Hexachloro-1,3-butadiene } \\
\left(\mathrm{C}_{4} \mathrm{Cl}_{6}\right) \text { (hexachlorobutaciene) }\end{array}$ \\
\hline 32105 & $124-48-1$ & $\begin{array}{l}\text { Dibromochloromethane }\left(\mathrm{CHBr}_{2} \mathrm{Cl}\right) \\
\text { (chlorodibromomethane) }\end{array}$ & 34396 & $67-72-1$ & $\begin{array}{l}\text { 1,1,1,2,2,2-Hexachloroethane }\left(\mathrm{C}_{2} \mathrm{Cl}_{6}\right) \\
\quad \text { (carbon hexachloride) }\end{array}$ \\
\hline 77651 & $106-93-4$ & $\begin{array}{l}\text { 1,2-Dibromoethane }\left(\mathrm{C}_{2} \mathrm{H}_{4} \mathrm{Br}_{2}\right) \\
\text { (ethylene dibromide, } \mathrm{EDB})\end{array}$ & 50005 & $994-05-8$ & $\begin{array}{l}\text { 2-Methoxy-2-methylbutane }\left(\mathrm{C}_{6} \mathrm{H}_{14} \mathrm{O}\right) \\
\text { (tert-amyl methyl ether, TAME) }\end{array}$ \\
\hline 34536 & $95-50-1$ & $\begin{array}{l}\text { 1,2-Dichlorobenzene }\left(\mathrm{C}_{6} \mathrm{H}_{4} \mathrm{Cl}_{2}\right) \\
\text { (o-dichlorobenzene) }\end{array}$ & 78032 & $1634-04-4$ & $\begin{array}{l}\text { 2-Methoxy-2-methylpropan: }\left(\mathrm{C}_{5} \mathrm{H}_{12} \mathrm{O}\right) \\
\text { (methyl tert-butyl ether, ITTE) }\end{array}$ \\
\hline 34566 & $541-73-1$ & $\begin{array}{l}\text { 1,3-Dichlorobenzene }\left(\mathrm{C}_{6} \mathrm{H}_{4} \mathrm{Cl}_{2}\right) \\
\quad(m \text {-dichlorobenzene })\end{array}$ & 34010 & $108-88-3$ & $\begin{array}{l}\text { Methylbenzene }\left(\mathrm{C}_{7} \mathrm{H}_{8}\right) \\
\text { (toluene) }\end{array}$ \\
\hline 34571 & $106-46-7$ & $\begin{array}{l}\text { 1,4-Dichlorobenzene }\left(\mathrm{C}_{6} \mathrm{H}_{4} \mathrm{Cl}_{2}\right) \\
\quad \text { (p-dichlorobenzene) }\end{array}$ & 77223 & $98-82-8$ & $\begin{array}{l}\text { (1-Methylethyl)benzene }\left(\mathrm{C}_{0} \mathrm{H}_{12}\right) \\
\text { (isopropylbenzene) }\end{array}$ \\
\hline 34668 & $75-71-8$ & $\begin{array}{l}\text { Dichlorodifluoromethane }\left(\mathrm{CCl}_{2} \mathrm{~F}_{2}\right) \\
\quad \text { (CFC 12) }\end{array}$ & 34696 & $91-20-3$ & Naphthalene $\left(\mathrm{C}_{10} \mathrm{H}_{8}\right)$ \\
\hline 34496 & $75-34-3$ & $\begin{array}{l}\text { 1,1-Dichloroethane }\left(\mathrm{C}_{2} \mathrm{H}_{4} \mathrm{Cl}_{2}\right) \\
\quad \text { (ethylidene chloride) }\end{array}$ & 81577 & $108-20-3$ & $\begin{array}{l}\text { 2,2'-oxybis[propane] }\left(\mathrm{C}_{6} \mathrm{H}_{14} \mathrm{O}\right) \\
\text { (diisopropyl ether, DIPE) }\end{array}$ \\
\hline 32103 & $107-06-2$ & $\begin{array}{l}\text { 1,2-Dichloroethane }\left(\mathrm{C}_{2} \mathrm{H}_{4} \mathrm{Cl}_{2}\right) \\
\text { (ethylene dichloride) }\end{array}$ & 34210 & $107-02-8$ & $\begin{array}{l}\text { 2-Propenal }\left(\mathrm{C}_{3} \mathrm{H}_{4} \mathrm{O}\right) \\
\quad \text { (acrolein) }\end{array}$ \\
\hline 34501 & $75-35-4$ & $\begin{array}{l}\text { 1,1-Dichloroethene }\left(\mathrm{C}_{2} \mathrm{H}_{2} \mathrm{Cl}_{2}\right) \\
\text { (vinylidene chloride) }\end{array}$ & 34215 & $107-13-1$ & $\begin{array}{l}\text { 2-Propenenitrile }\left(\mathrm{C}_{3} \mathrm{H}_{3} \mathrm{~N}\right) \\
\quad \text { (acrylonitrile) }\end{array}$ \\
\hline 77093 & $156-59-2$ & $\begin{array}{l}\text { cis-1,2-Dichloroethene }\left(\mathrm{C}_{2} \mathrm{H}_{2} \mathrm{Cl}_{2}\right) \\
\text { ((Z)-1,2-dichloroethene) }\end{array}$ & 77224 & $103-65-1$ & $\begin{array}{l}n \text {-Propylbenzene }\left(\mathrm{C}_{9} \mathrm{H}_{12}\right) \\
\text { (1-phenylpropane) }\end{array}$ \\
\hline 34546 & $156-60-5$ & $\begin{array}{l}\text { trans-1,2-Dichloroethene }\left(\mathrm{C}_{2} \mathrm{H}_{2} \mathrm{Cl}_{2}\right) \\
\quad((\mathrm{E})-1,2 \text {-dichloroethene })\end{array}$ & 34475 & $127-18-4$ & $\begin{array}{l}\text { Tetrachloroethene }\left(\mathrm{C}_{2} \mathrm{Cl}_{4}\right) \\
\text { (perchloroethene, } \mathrm{PCE} \text { ) }\end{array}$ \\
\hline 34423 & $75-09-2$ & $\begin{array}{l}\text { Dichloromethane }\left(\mathrm{CH}_{2} \mathrm{Cl}_{2}\right) \\
\text { (methylene chloride) }\end{array}$ & 32102 & $56-23-5$ & $\begin{array}{l}\text { Tetrachloromethane }\left(\mathrm{CCl}_{4}\right) \\
\text { (carbon tetrachloride) }\end{array}$ \\
\hline
\end{tabular}


Table 1. Volatile organic compounds measured by the U.S. Geological Survey National Water-Quality Assessmer* Program-Continued

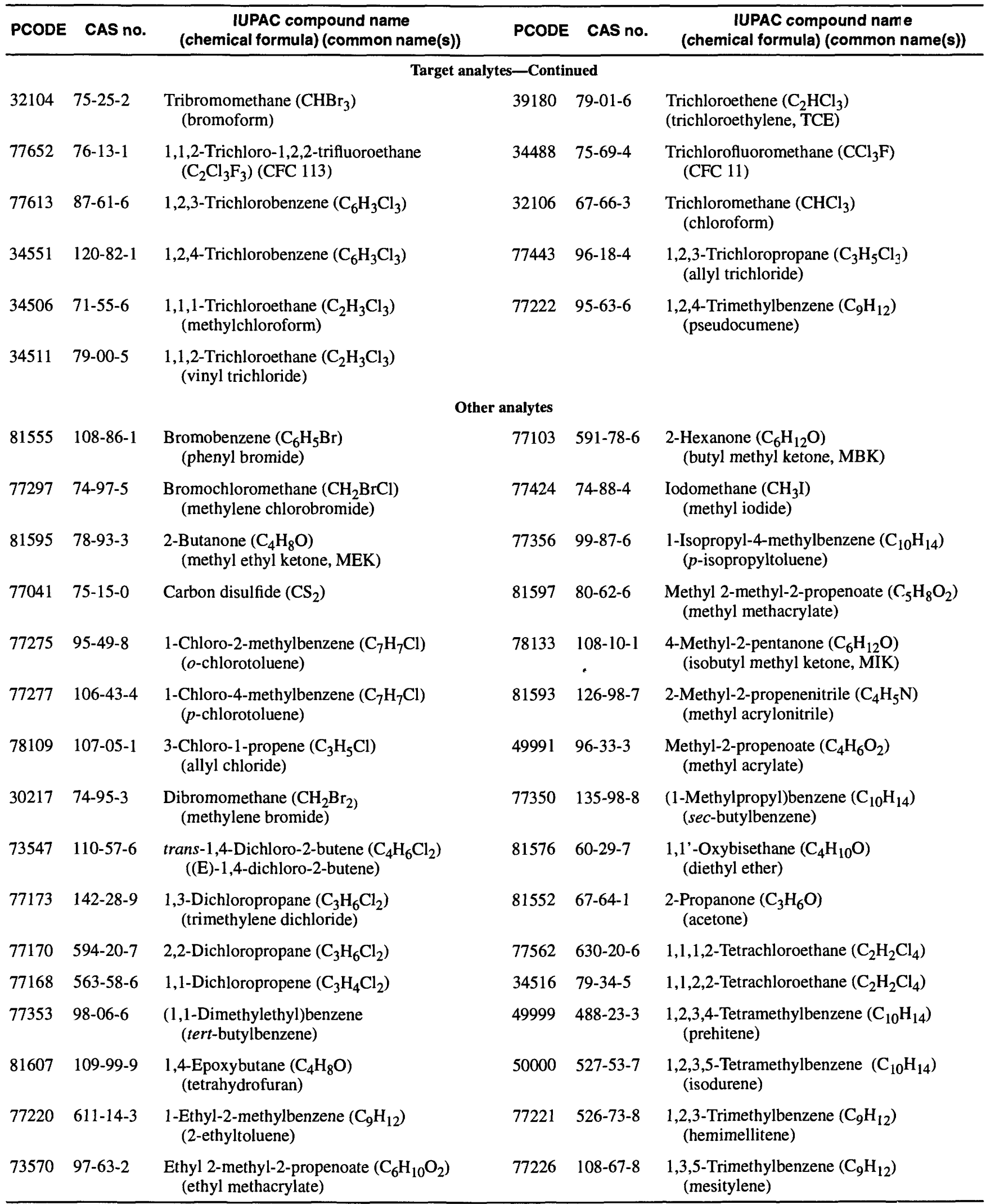


Other toxicity information for VOCs include: (1) noobserved effective concentration (NOEC), the highest concentration at which observed effects are not found or are not statistically significant; (2) lowest-observed effective concentration (LOEC), the lowest concentration producing a statistically significant effect; and (3) maximum-acceptable toxicant concentration, the chronic value representing the hypothetical threshold concentration that is the geometric mean between the NOEC and LOEC concentrations.

For most VOCs, toxicity information is available for only one or two test species. AQUIRE had no information for 13 NAWQA target analytes and 13 other analytes (table 2 ).

\section{Retrieval from AQUIRE}

Selected toxicity information was retrieved from AQUIRE by specifying the VOC and ancillary information including: chemical name recognized by the International Union of Pure and Applied Chemistry and common name, Chemical Abstracts registry number, taxonomic classification (latin and common name) of test species, number of studies (n) performed on each VOC, duration of exposure, concentration of the endpoint (for example, $\mathrm{LC}_{50}$ ), measured effect code that qualifies the documentation of test procedures, and source number indicating the study reference. Not all entries in AQUIRE have the ancillary information that were specified in the retrievals.

\section{Criteria for Compilation of Information}

To be included in this report, information from AQUIRE was reviewed to determine if the following two criteria were met: (1) studies must have been conducted in freshwater, and (2) studies must have complete or moderately complete documentation of test procedures. A number of studies using the same species, duration, and endpoint were available for some VOCs. In these instances, only the two studies that reported the lowest concentrations that had an effect were included in the compilation at the end of this report in the Aquatic Toxicity Information for Selected Volatile Organic Compounds section (table 5) and in the References Containing Aquatic Toxicity Information section (table 6).
Table 2. Volatile organic compounds with no available aquatic toxicity information

[IUPAC, International Union of Pure and Applied Chem:stry]

\begin{tabular}{l} 
IUPAC Compound Name \\
\hline \multicolumn{1}{c}{ Target analytes } \\
Bromodichloromethane \\
Bromoethene \\
Chloroethane \\
Chloroethene \\
Dichlorodifluoromethane \\
1,1-Dichloroethane \\
cis-1,2-Dichloroethene \\
cis-1,3-Dichloropropene \\
trans-1,3-Dichloropropene \\
2-Ethoxy-2-methylpropane \\
2-Methoxy-2-methylbutane \\
1,1,2-Trichloro-1,2,2-trifluoroethane \\
Trichlorofluoromethane \\
Bromor analytes \\
1,2,3-Trimethylbenzene \\
1-2,-Dichloropropane \\
1,-2,3,4-Tetramethylbenzene \\
Eibromomethane
\end{tabular}

\section{Lowest Concentrations of Volatile Organic Compounds that Affect a Specirs}

Some species are more sensitive to certain contaminants than others and are affected at lower concentrations. For this reason, aquatic toxicity information was reviewed for the lowest concentration that had any effect on any species. This information is summarized in table 3. Some compounds are acutely toxic to a species over a relatively narrow concentration range. When this occurs, the lowest concentratic $\eta$ that affects the species is reported as a range rather than a single value. 
Table 3. Lowest concentrations of volatile organic compounds that affect a species

[IUPAC, International Union of Pure and Applied Chemistry; when available, the lowest concentration is given in the following order of precedence: MATC, maximum-acceptable toxicant concentration; LOEC, lowest-observed effective concentration; $\mathrm{EC}_{50}$, median effective concentration; $\mathrm{LC}_{50}$, me tian lethal concentration; --, not reported; nv, no value; <, less than; >, greater than; endpoint effect: AVO, avoidance; BEH, behavior; BMS, biomass; CLR, chlorophyll; GRO, growth; IMM, immobilization; MOR, mortality, effect expressed as percentage of death; PSE, photosynthesis; PTR, phototactic response; REP, reproduction; TER, teratogenesis]

\begin{tabular}{|c|c|c|c|c|c|c|c|c|}
\hline IUPAC compound name & $\begin{array}{c}\text { Taxo- } \\
\text { nomic } \\
\text { classifi- } \\
\text { cation }\end{array}$ & $\begin{array}{l}\text { Genus, species/ } \\
\text { Common name }\end{array}$ & \multicolumn{4}{|c|}{ Lowest concentration (micrograms per liter) } & $\begin{array}{l}\text { End- } \\
\text { point } \\
\text { effect }\end{array}$ & $\begin{array}{c}\text { Duration } \\
\text { of test } \\
\text { (hours) }\end{array}$ \\
\hline
\end{tabular}

\section{Target analytes}

\begin{tabular}{|c|c|c|c|c|c|c|c|c|}
\hline Benzene & fish & $\begin{array}{l}\text { Pimephales promelas/ } \\
\text { Fathead minnow }\end{array}$ & nv & 17,200 & -- & -- & GRO & 168 \\
\hline Bromomethane & fish & $\begin{array}{l}\text { Oryzias latipes/ } \\
\text { Medaka, high-eyes }\end{array}$ & nv & nv & 400 & -- & BEH & 96 \\
\hline$n$-Butylbenzene & insects & $\begin{array}{l}\text { Daphnia magnal } \\
\text { Water flea }\end{array}$ & 490 & -- & -- & -- & IMM & 48 \\
\hline Chlorobenzene & insects & $\begin{array}{l}\text { Daphnia magnal } \\
\text { Water flea }\end{array}$ & nv & nv & 585 & -- & IMM & 48 \\
\hline Chloromethane & fish & $\begin{array}{l}\text { Lepomis macrochirus/ } \\
\text { Bluegill }\end{array}$ & nv & nv & nv & 550,000 & MOR & 96 \\
\hline Dibromochloromethane & fish & $\begin{array}{l}\text { Cyprinus carpiol } \\
\text { Common, mirror, colored, } \\
\text { carp }\end{array}$ & nv & nv & nv & 34,000 & MOR & $72-120$ \\
\hline 1,2-Dichlorobenzene & insects & $\begin{array}{l}\text { Daphnia magnal } \\
\text { Water flea }\end{array}$ & nv & nv & 550 & -- & REP & 336 \\
\hline 1,3-Dichlorobenzene & fish & $\begin{array}{l}\text { Oncorhynchus mykiss/ } \\
\text { Rainbow trout, donaldson } \\
\text { trout }\end{array}$ & $555-1,040$ & -- & -- & -- & GRO & 768 \\
\hline 1,4-Dichlorobenzene & fish & $\begin{array}{l}\text { Oncorhynchus mykiss/ } \\
\text { Rainbow trout, donaldson } \\
\text { trout }\end{array}$ & $565-1,040$ & -- & -- & -- & GRO & 768 \\
\hline $\begin{array}{l}\text { Dichlorobenzene } \\
\text { (all isomers) }\end{array}$ & fish & $\begin{array}{l}\text { Brachydanio reriol } \\
\text { Zebra danio, zebrafish }\end{array}$ & nv & nv & nv & 10,000 & MOR & 48 \\
\hline trans-1,2-Dichloroethene & insects & $\begin{array}{l}\text { Daphnia magnal } \\
\text { Water flea }\end{array}$ & nv & nv & nv & 220,000 & MOR & 48 \\
\hline Dichloromethane & $\begin{array}{l}\text { amphib- } \\
\text { ians }\end{array}$ & $\begin{array}{l}\text { Rana catesbeianal } \\
\text { Bullfrog }\end{array}$ & nv & nv & 17,780 & -- & TER & 192 \\
\hline 1,2-Dichloropropane & fish & $\begin{array}{l}\text { Pimephales promelas/ } \\
\text { Fathead minnow }\end{array}$ & $6,000-11,000$ & -- & - & -- & GRO & 768 \\
\hline 1,3-Dichloropropene & insects & $\begin{array}{l}\text { Daphnia magnal } \\
\text { Water flea }\end{array}$ & nv & nv & 90 & -- & IMM & 48 \\
\hline
\end{tabular}


Table 3. Lowest concentrations of volatile organic compounds that affect a species-Continued

\begin{tabular}{|c|c|c|c|c|c|c|c|c|}
\hline IUPAC compound name & $\begin{array}{l}\text { Taxo- } \\
\text { nomic } \\
\text { classifi- } \\
\text { cation }\end{array}$ & $\begin{array}{l}\text { Genus, species } \\
\text { Common name }\end{array}$ & \multicolumn{4}{|c|}{ Lowest concentration (micrograms per liter) } & $\begin{array}{l}\text { End- } \\
\text { point } \\
\text { ef sct }\end{array}$ & $\begin{array}{c}\text { Duration } \\
\text { of test } \\
\text { (hours) }\end{array}$ \\
\hline 1,2-Dimethylbenzene & fish & $\begin{array}{l}\text { Oncorhynchus kisutch/ } \\
\text { Coho salmon, silver } \\
\text { salmon }\end{array}$ & nv & nv & 600 & -- & AVO & 1 \\
\hline 1,4-Dimethylbenzene & plants & $\begin{array}{l}\text { Selenastrum } \\
\quad \text { capricornutum/ } \\
\text { Green algae }\end{array}$ & nv & nv & 3,200 & -- & GRO & 72 \\
\hline Ethenylbenzene & fish & $\begin{array}{l}\text { Oncorhynchus mykiss/ } \\
\text { Rainbow trout, donaldson } \\
\text { trout }\end{array}$ & nv & nv & nv & 2,500 & MOR & 24 \\
\hline Ethylbenzene & insects & $\begin{array}{l}\text { Daphnia magnal } \\
\text { Water flea }\end{array}$ & nv & nv & 1,810 & -- & IMM & 24 \\
\hline $\begin{array}{l}\text { 1,1,2,3,4,4-Hexachloro-1,3- } \\
\text { butadiene }\end{array}$ & fish & $\begin{array}{l}\text { Pimephales promelas/ } \\
\text { Fathead minnow }\end{array}$ & $6.5-13$ & -- & -- & -- & GRO & 768 \\
\hline $1,1,1,2,2,2-$ Hexachloroethane & fish & $\begin{array}{l}\text { Oncorhynchus mykiss/ } \\
\text { Rainbow trout, donaldson } \\
\text { trout }\end{array}$ & $67-207$ & -- & -- & -- & GRO & 768 \\
\hline Naphthalene & insects & $\begin{array}{l}\text { Daphnia magnal } \\
\text { Water flea }\end{array}$ & $\mathrm{nv}$ & nv & 690 & -- & PTR & 2 \\
\hline 2,2-Oxybis[propane] & fish & $\begin{array}{l}\text { Pimephales promelas/ } \\
\text { Fathead minnow }\end{array}$ & $\mathrm{nv}$ & nv & nv & 91,700 & MOR & 96 \\
\hline 2-Propenal & insects & $\begin{array}{l}\text { Daphnia magnal } \\
\text { Water flea }\end{array}$ & nv & nv & 51 & -- & IMM & 48 \\
\hline 2-Propenenitrile & insects & $\begin{array}{l}\text { Daphnia magnal } \\
\text { Water flea }\end{array}$ & nv & nv & 10,950 & -- & IMM & 48 \\
\hline$n$-Propylbenzene & plants & $\begin{array}{l}\text { Selenastrum } \\
\text { capricornutum/ } \\
\text { Green algae }\end{array}$ & nv & $\mathrm{nv}$ & 1,800 & -- & GPO & 72 \\
\hline Tetrachloroethene & fish & $\begin{array}{l}\text { Oncorhynchus mykiss/ } \\
\text { Rainbow trout, donaldson } \\
\text { trout }\end{array}$ & $500-1,400$ & - & -- & -- & GPO & 768 \\
\hline Tetrachloromethane & fish & $\begin{array}{l}\text { Pimephales promelas/ } \\
\text { Fathead minnow }\end{array}$ & 52,100 & -- & -- & -- & MOR & 168 \\
\hline Tribromomethane & plants & $\begin{array}{l}\text { Selenastrum } \\
\text { capricornutum/ } \\
\text { Green algae }\end{array}$ & $\mathrm{nv}$ & nv & 38,600 & -- & CIJR & 96 \\
\hline
\end{tabular}


Table 3. Lowest concentrations of volatile organic compounds that affect a species-Continued

\begin{tabular}{|c|c|c|c|c|c|c|c|c|}
\hline IUPAC compound name & $\begin{array}{l}\text { Taxo- } \\
\text { nomic } \\
\text { classifi- } \\
\text { cation }\end{array}$ & $\begin{array}{l}\text { Genus, species/ } \\
\text { Common name }\end{array}$ & \multicolumn{4}{|c|}{ Lowest concentration (micrograms per liter) } & $\begin{array}{l}\text { End- } \\
\text { point } \\
\text { effect }\end{array}$ & $\begin{array}{c}\text { Duration } \\
\text { of test } \\
\text { (hours) }\end{array}$ \\
\hline 1,2,3-Trichlorobenzene & insects & $\begin{array}{l}\text { Daphnia magnal } \\
\text { Water flea }\end{array}$ & nv & nv & 200 & -- & REP & 336 \\
\hline 1,1,1-Trichloroethane & fish & $\begin{array}{l}\text { Pimephales promelas/ } \\
\text { Fathead minnow }\end{array}$ & nv & nv & 11,100 & -- & IMM & 72 \\
\hline 1,1,2-Trichloroethane & fish & $\begin{array}{l}\text { Oncorhynchus mykiss/ } \\
\text { Rainbow trout, donaldson } \\
\text { trout }\end{array}$ & $\begin{array}{l}6,000- \\
14,800\end{array}$ & -- & -- & -- & GRO & 768 \\
\hline Trichloromethane & $\begin{array}{l}\text { amphib- } \\
\text { ians }\end{array}$ & $\begin{array}{l}\text { Hyla cruciferl } \\
\text { Spring peeper }\end{array}$ & nv & nv & 270 & -- & TER & 168 \\
\hline 1,2,3-Trichloropropane & fish & $\begin{array}{l}\text { Pimephales promelas/ } \\
\text { Fathead minnow }\end{array}$ & $\mathrm{nv}$ & $\mathrm{nv}$ & nv & 66,500 & MOR & 96 \\
\hline 1,2,4-Trimethylbenzene & insects & $\begin{array}{l}\text { Daphnia magnal } \\
\text { Water flea }\end{array}$ & nv & nv & 3,600 & -- & IMM & 48 \\
\hline \multicolumn{9}{|c|}{ Other analytes } \\
\hline Bromochloromethane & fish & $\begin{array}{l}\text { Cyprinus carpiol } \\
\text { Common, mirror, colored, } \\
\text { carp }\end{array}$ & nv & nv & nv & 67,000 & MOR & $72-120$ \\
\hline 1-Chloro-4-methylbenzene & fish & $\begin{array}{l}\text { Brachydanio reriol } \\
\text { Zebra danio, zebrafish }\end{array}$ & nv & nv & nv & 4,400 & MOR & 672 \\
\hline 3-Chloro-1-propene & $\begin{array}{l}\text { amphib- } \\
\text { ians }\end{array}$ & $\begin{array}{l}\text { Xenopus laevis/ } \\
\text { Clawed toad }\end{array}$ & nv & nv & nv & 340 & MOR & 48 \\
\hline 1,3-Dichloropropane & fish & $\begin{array}{l}\text { Pimephales promelas/ } \\
\text { Fathead minnow }\end{array}$ & $\begin{array}{l}8,000- \\
16,000\end{array}$ & -- & -- & -- & GRO & 768 \\
\hline (1,1-Dimethylethyl)benzene & insects & $\begin{array}{l}\text { Daphnia magnal } \\
\text { Water flea }\end{array}$ & nv & nv & nv & 41,000 & MOR & 24 \\
\hline 1,4-Epoxybutane & fish & $\begin{array}{l}\text { Carassius auratus/ } \\
\text { Goldfish }\end{array}$ & $\mathrm{nv}$ & nv & $\mathrm{nv}$ & $2,400,000$ & MOR & 48 \\
\hline Ethenylethanoate & fish & $\begin{array}{l}\text { Carassius auratus/ } \\
\text { Goldfish }\end{array}$ & nv & nv & nv & 42,330 & MOR & 24 \\
\hline 2-Hexanone & fish & $\begin{array}{l}\text { Pimephales promelas/ } \\
\text { Fathead minnow }\end{array}$ & nv & nv & nv & 428,000 & MOR & 96 \\
\hline 1-Isopropyl-4-methylbenzene & plants & $\begin{array}{l}\text { Skeletonema costatum/ } \\
\text { Diatom }\end{array}$ & nv & nv & 22,000 & -- & PSE & 96 \\
\hline
\end{tabular}


Table 3. Lowest concentrations of volatile organic compounds that affect a species-Continued

\begin{tabular}{|c|c|c|c|c|c|c|c|c|}
\hline IUPAC compound name & $\begin{array}{l}\text { Taxo- } \\
\text { nomic } \\
\text { classifi- } \\
\text { cation }\end{array}$ & $\begin{array}{l}\text { Genus, species } \\
\text { Common name }\end{array}$ & \multicolumn{4}{|c|}{ Lowest concentration (micrograms per liter) } & $\begin{array}{l}\text { End- } \\
\text { pcint } \\
\text { effect }\end{array}$ & $\begin{array}{c}\text { Duration } \\
\text { of test } \\
\text { (hours) }\end{array}$ \\
\hline $\begin{array}{l}\text { Methyl 2-methyl-2- } \\
\text { propenoate }\end{array}$ & fish & $\begin{array}{l}\text { Pimephales promelas/ } \\
\text { Fathead minnow }\end{array}$ & $\mathrm{nv}$ & nv & nv & 130,000 & MCR & 96 \\
\hline Methyl-2-propenoate & fish & $\begin{array}{l}\text { Osteichthyes/ } \\
\text { Bony fish class }\end{array}$ & $\mathrm{nv}$ & nv & $\mathrm{nv}$ & 5,000 & MCR & 72 \\
\hline 1,1'-Oxybisethane & fish & $\begin{array}{l}\text { Oryzias latipes/ } \\
\text { Medaka, high-eyes }\end{array}$ & $\mathrm{nv}$ & nv & nv & $>1,000,000$ & MCR & 24 \\
\hline 1,1,2,2-Tetrachloroethane & fish & $\begin{array}{l}\text { Oncorhynchus mykiss/ } \\
\text { Rainbow trout, donaldson } \\
\text { trout }\end{array}$ & $1,400-4,000$ & -- & -- & -- & GRO & 768 \\
\hline 1,3,5-Trimethylbenzene & insects & $\begin{array}{l}\text { Daphnia magnal } \\
\text { Water flea }\end{array}$ & nv & $\mathrm{nv}$ & 6,011 & - & IMM & 48 \\
\hline
\end{tabular}

\section{WATER-QUALITY CRITERIA AND GUIDELINES FOR VOLATILE ORGANIC COMPOUNDS}

Water-quality criteria and guidelines for 39 VOCs (table 4) measured by the USGS have been established by USEPA (U.S. Environmental Protection Agency, 1996a) and Canada (Canadian Council of Resource and Environment Ministers, 1991) to protect freshwater biota. When toxicity information is insufficient to develop criteria, USEPA uses values equal to the LOEC. Canadian water-quality guidelines, which are nonenforceable guidelines that may provide the basis for Provincial Standards or Objectives (Richard Aucoin, Environment Canada, oral commun., 1997) are lower than the USEPA criteria. This may be attributed to procedures for determining the values. Canadian Water-Quality Guidelines, which " *** are set at such values as to protect all forms of aquatic life and all aspects of the aquatic life cycles" (Canadian Council of Resource and Environment Ministers, 1991), contrasts with the USEPA approach in which "*** protection of all species at all times and places is not deemed necessary" (Stephan and others, 1985). Canadian guidelines are derived from the most sensitive of the following: (1) "no negative effect" data ol+ained from life-stage tests of chronic toxicity; (2) thresholds for the tainting of fish flesh; or (3) concentrations in the water that would result in acceptable concentrations in the edible portions of marketable fish (or the protection of natural consumers). If sufficient chronic toxicity data are not available, Canadian guidelines are based on short-term toxicity data ( 96 hour $\mathrm{LC}_{50}$ ) of the most sensitive species mutliplied by application factors of 0.05 for nonpersistent effects and 0.01 for persistent effects.

The USEPA's aquatic-life criteria are nonenforceable scientific recommendations that may provide the basis for State standards. Acute and chronic criteria issued under the 1985 guidelines (Stephar and others, 1985) are the criterion maximum concentration (CMC) and the criterion continuous concentratior (CCC), respectively. The acute and chronic criteria are based on the highest concentration of a pollutant that freshwater aquatic organisms can be exposed tc for an established period of time without deleterious effects1 hour for CMC and 4 days for CCC. Water-quality criteria are based on guidelines establishec' using extensive data requirements and complex math matical derivations. 
Table 4. Water-quality criteria and guidelines for volatile organic compounds

[IUPAC, International Union of Pure and Applied Chemistry; USEPA, U.S. Environmental Protection Agency; all values are in micrograms Far liter; --, criteria does not exist]

\begin{tabular}{|c|c|c|c|}
\hline IUPAC compound name & $\begin{array}{c}\text { USEPA } \\
\text { freshwater acute } \\
\text { criteria/guidelines }\end{array}$ & $\begin{array}{c}\text { USEPA } \\
\text { freshwater chronic } \\
\text { criteria/guidelines }^{1}\end{array}$ & $\begin{array}{c}\text { Canac'ian } \\
\text { water-q'ality } \\
\text { guideli-es }^{2}\end{array}$ \\
\hline \multicolumn{4}{|c|}{ Target analytes } \\
\hline Benzene & 5,300 & -- & 370 \\
\hline Bromodichloromethane & 11,000 & -- & -- \\
\hline Bromomethane & 11,000 & -- & -- \\
\hline Chlorobenzene & 250 & 50 & 15 \\
\hline Chloroethane & ${ }^{3} 860,000$ & ${ }^{4} 230,000$ & -- \\
\hline Dibromochloromethane & 11,000 & -- & -- \\
\hline 1,2-Dichlorobenzene & 1,120 & 763 & 2.5 \\
\hline 1,3-Dichlorobenzene & 1,120 & 763 & 2.5 \\
\hline 1,4-Dichlorobenzene & -- & -- & 4 \\
\hline Dichlorodifluoromethane & 11,000 & -- & -- \\
\hline 1,2-Dichloroethane & 118,000 & 20,000 & 100 \\
\hline 1,1-Dichloroethene & 11,600 & -- & -- \\
\hline cis-1,2-Dichloroethene & 11,600 & -- & -- \\
\hline trans-1,2-Dichloroethene & 11,600 & - & -- \\
\hline Dichloromethane & 11,000 & -- & 98 \\
\hline cis-1,3-Dichloropropene & 6,600 & 244 & -- \\
\hline trans-1,3-Dichloropropene & 6,600 & 244 & -- \\
\hline Ethylbenzene & 32,000 & -- & 90 \\
\hline 1,1,2,3,4,4-Hexachloro-1,3-butadiene & 90 & 9.3 & .1 \\
\hline 1,1,1,2,2,2-Hexachloroethane & 980 & 540 & -- \\
\hline Methylbenzene & 17,500 & -- & 2 \\
\hline Naphthalene & 2,300 & 620 & -- \\
\hline 2-Propenal & 68 & 21 & 1,070 \\
\hline 2-Propenenitrile & 7,550 & 2,600 & -- \\
\hline Tetrachloroethene & - & -- & 110 \\
\hline Tetrachloromethane & 35,200 & -- & 13 \\
\hline Tribromomethane & 11,000 & -- & - \\
\hline 1,2,3-Trichlorobenzene & -- & -- & .9 \\
\hline 1,2,4-Trichlorobenzene & 250 & 50 & .5 \\
\hline 1,1,1-Trichloroethane & 18,000 & -- & -- \\
\hline 1,1,2-Trichloroethane & 18,000 & 9,400 & -. \\
\hline Trichloroethene & 45,000 & 21,900 & 20 \\
\hline Trichlorofluoromethane & 11,000 & - & -- \\
\hline Trichloromethane & 28,900 & 1,240 & 2 \\
\hline \multicolumn{4}{|c|}{ Other analytes } \\
\hline Bromochloromethane & 11,000 & -- & -- \\
\hline Carbon disulfide & -- & 2 & -- \\
\hline 3-Chloro-1-propene & ${ }^{3} 860,000$ & ${ }^{4} 230,000$ & -- \\
\hline 1,1,1,2-Tetrachloroethane & 9,320 & - & -- \\
\hline 1,1,2,2-Tetrachloroethane & 9,320 & 2,400 & -- \\
\hline
\end{tabular}

${ }^{1}$ U.S. Environmental Protection Agency, 1996b.

${ }^{2}$ Canadian Council of Resource and Environment Ministers, 1991

${ }^{3} 1$-hour average, freshwater acute water-quality criteria.

4-day average, freshwater chronic water-quality criteria. 


\section{REFERENCES CITED}

Canadian Council of Resource and Environment Ministers, 1991, Canadian water quality guidelines: Ottawa, Ontario, Environment Canada, Inland Waters Dictorate, Water Quality Branch (updated May 1996) [variously paged].

Eichelberger, J.W., and Budde, W.L., 1989, Method 524.2. Measurement of purgeable organic compounds in water by capillary column gas chromatography/mass spectrometry, revision 4.0: Cincinnati, Ohio, U.S. Environmental Protection Agency, Environmental Monitoring Systems Latoratory Office of Research and Development, p. 283-320.

Gilliom, R.J., Alley, W.M., and Gurtz, M.E., 1995, Design of the National Water-Quality Assessment ProgramOccurrence and distribution of water-quality conditions: U.S. Geological Survey Circular 1112, $33 \mathrm{p}$.

Lopes, T.J., and Price, C.V., 1997, Study plan for urban stream indicator sites of the National Water-Quality Assessment Program: U.S. Geological Survey OpenFile Report 97-25, 15 p.

Rand, G.M., and Petrocelli, S.R., 1985, Fundamentals of aquatic toxicology: Washington, Hemisphere Publishing Corporation, $666 \mathrm{p}$.
Rose, D.L., and Schroeder, M.P., 1994, Methoc's of analysis by the U.S. Geological Survey National W'ater-Quality Laboratory-Determination of volatile organic compounds in water by purge and trap capillary gas chromatography/mass spectrometry: U.S. Geological Survey Open-File Report 94-708, 26 p.

Squillace, P.J., and Price, C.V., 1996, Urban land-use study plan for National Water-Quality Assessmont: U.S. Geological Survey Open-File Report 96-217, 19 p.

Stephan, C.E., Mount, D.I., Hansen, D.J., Gen+ile, J.H., Chapman, G.A., and Brungs, W.A., 1985, Guidelines for deriving numerical national water-quality criteria for the protection of aquatic organisms ard their uses: Duluth, Minnesota, U.S. Environmental Protection Agency, Office of Research and Developnent, 98 p.

U.S. Environmental Protection Agency, 1996r、AQUatic toxicity Information REtrieval data base (AQUIRE): Duluth, Minnesota, U.S. Environmental Protection Agency, Environmental Research Laborctory, data retrieved May 1996 (electronic data files).

-1996b, Integrated Risk Information Sy'stem (IRIS): Research Triangle Park, N.C., U.S. Environmental Protection Agency, Office of Health and Environmental Assessment, electronic data files (Decerrber 1996 update). 
AQUATIC TOXICITY INFORMATION 


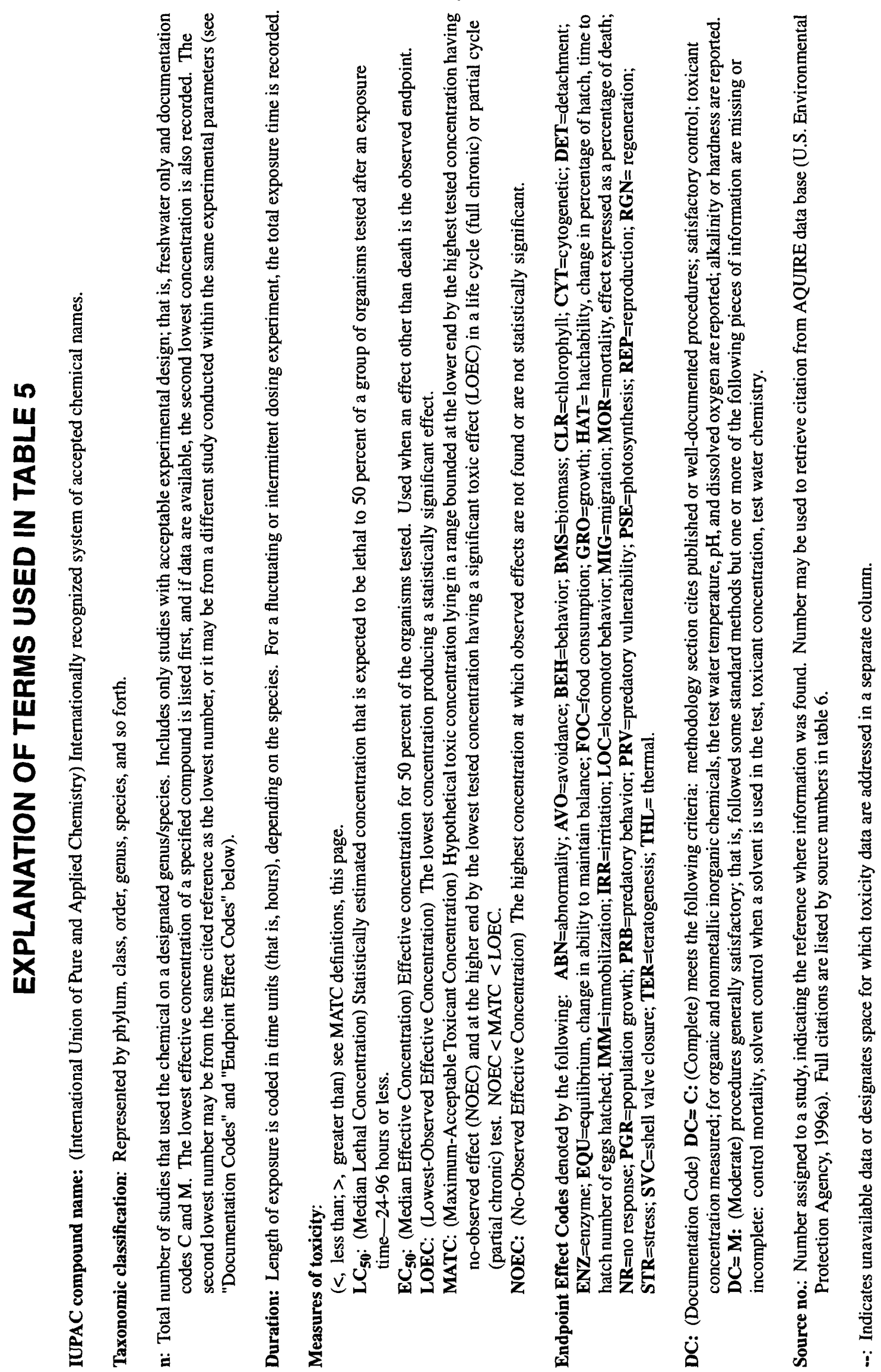




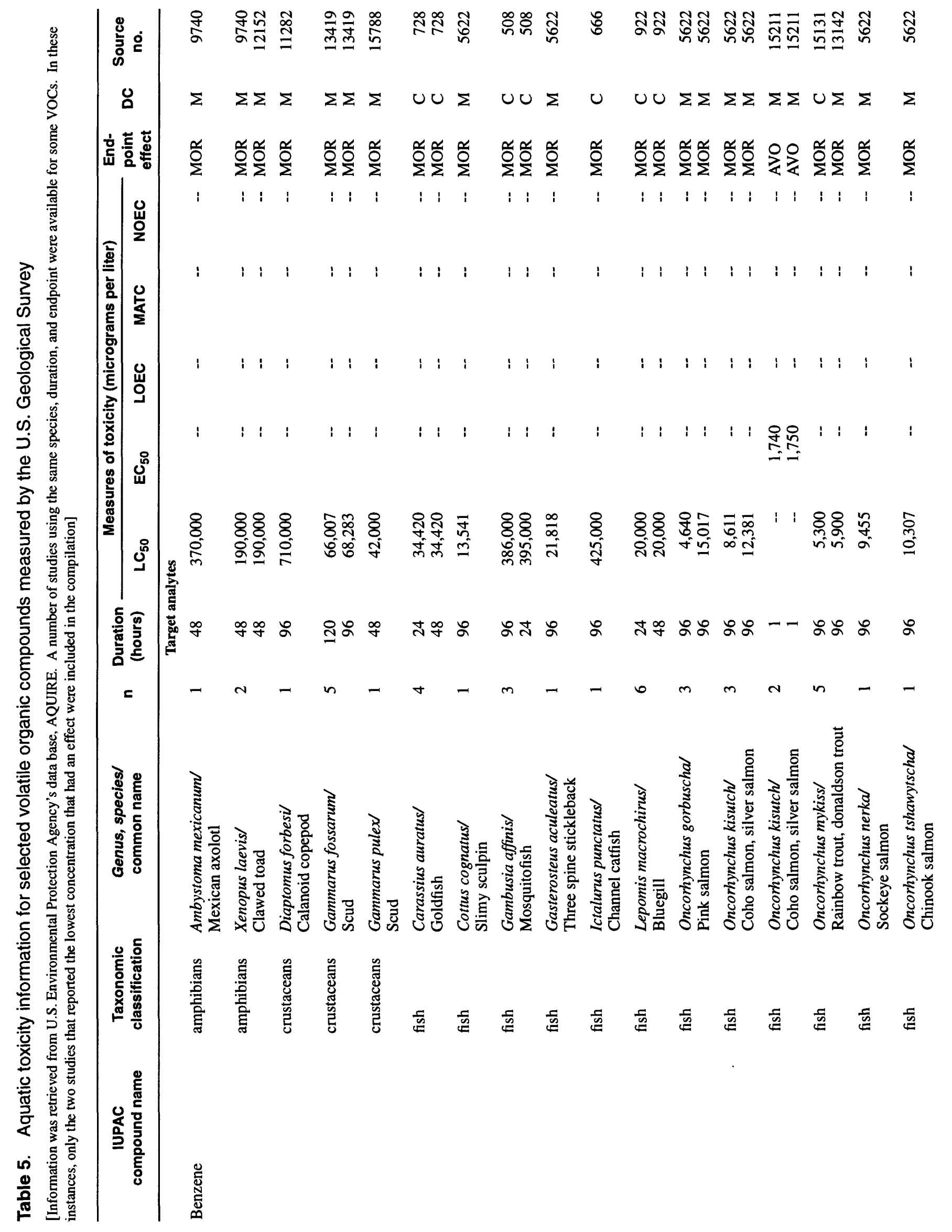




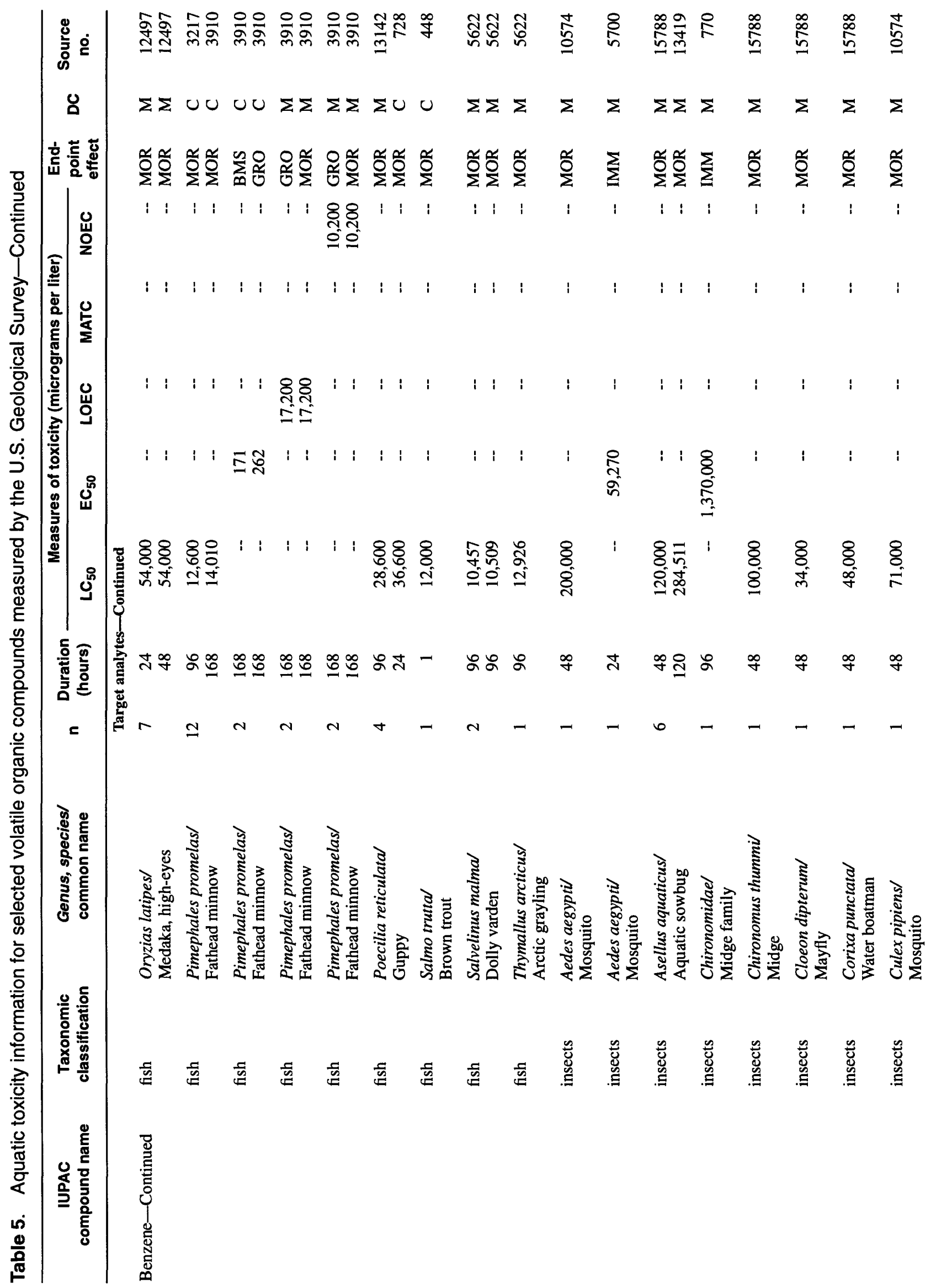




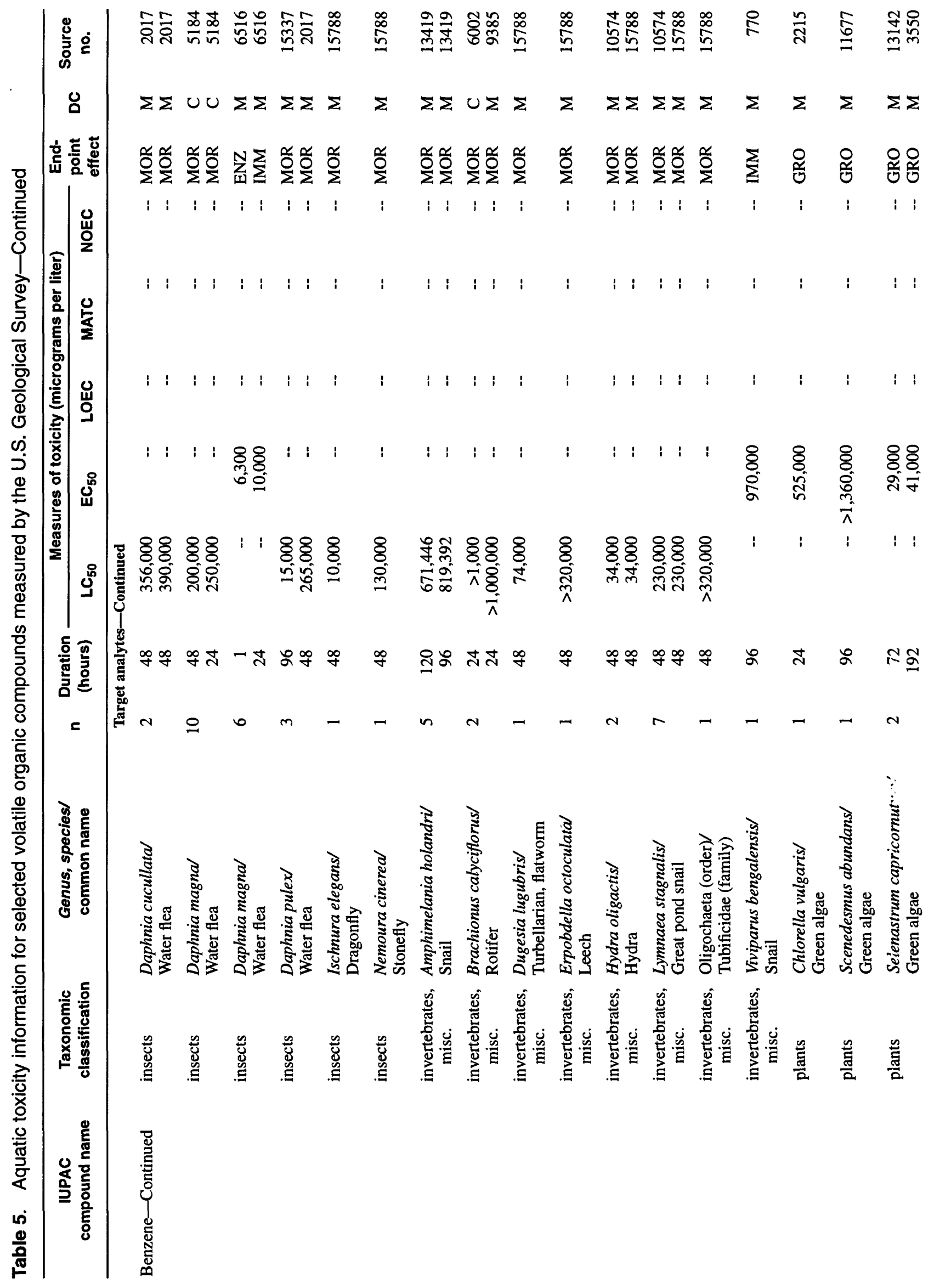




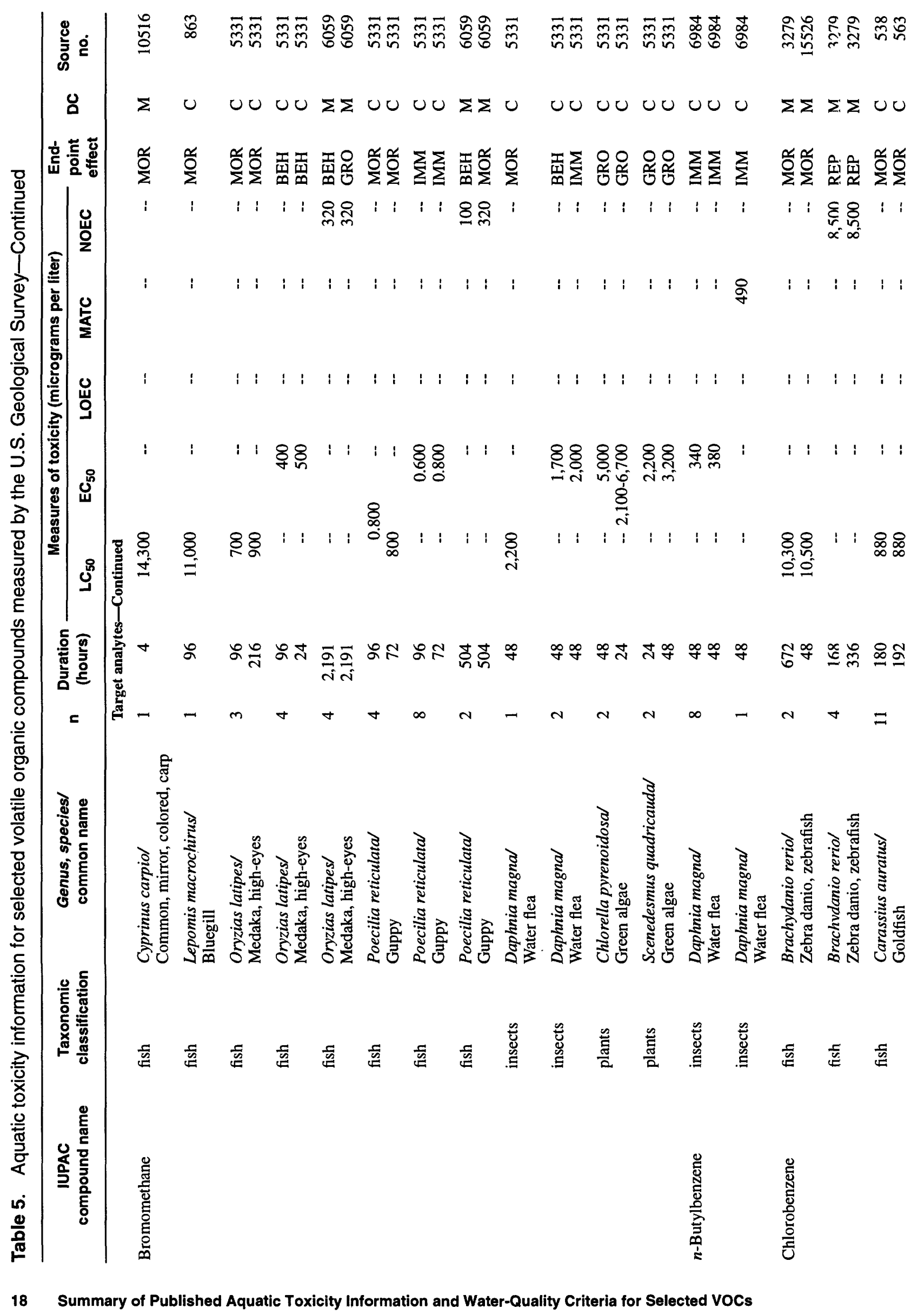




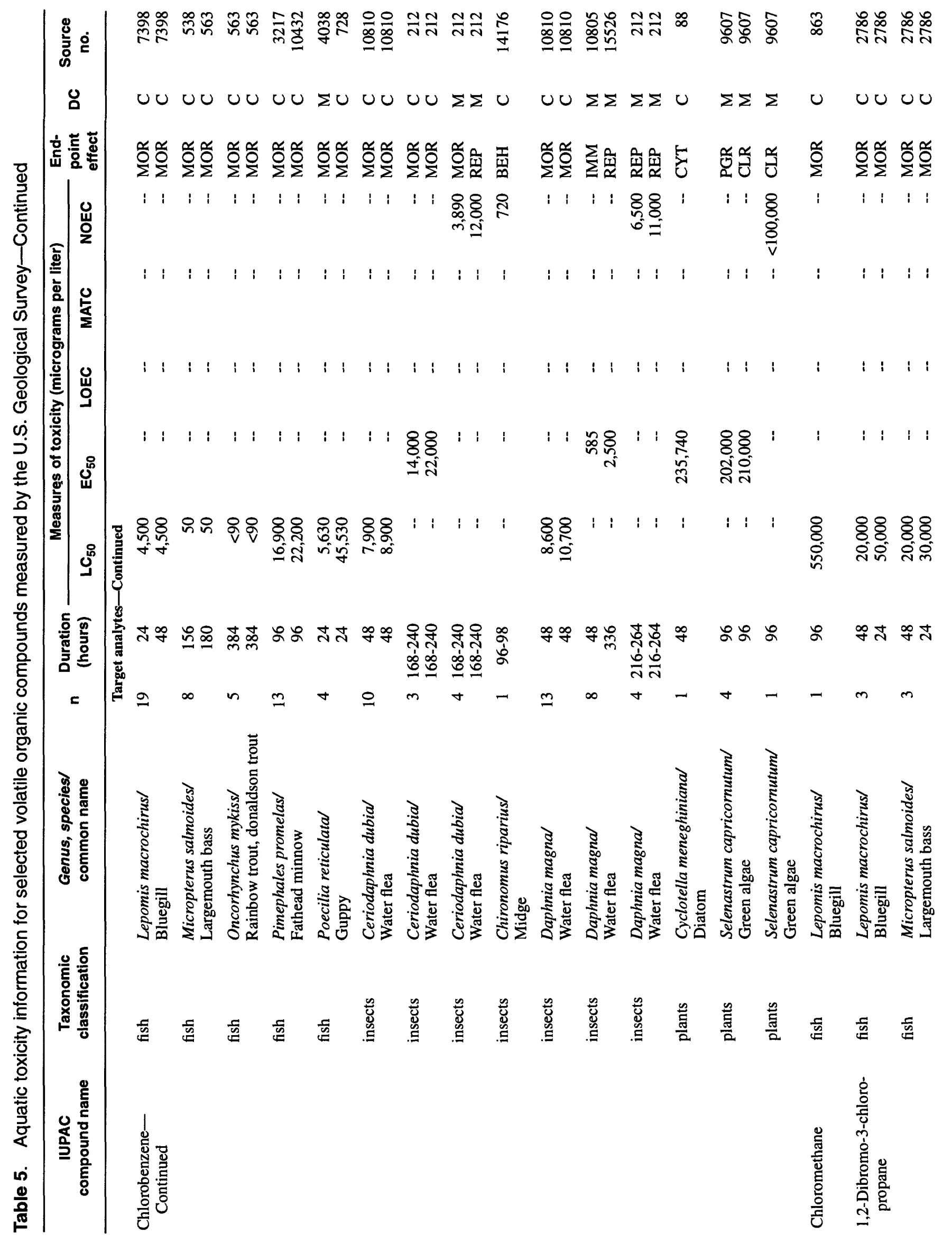




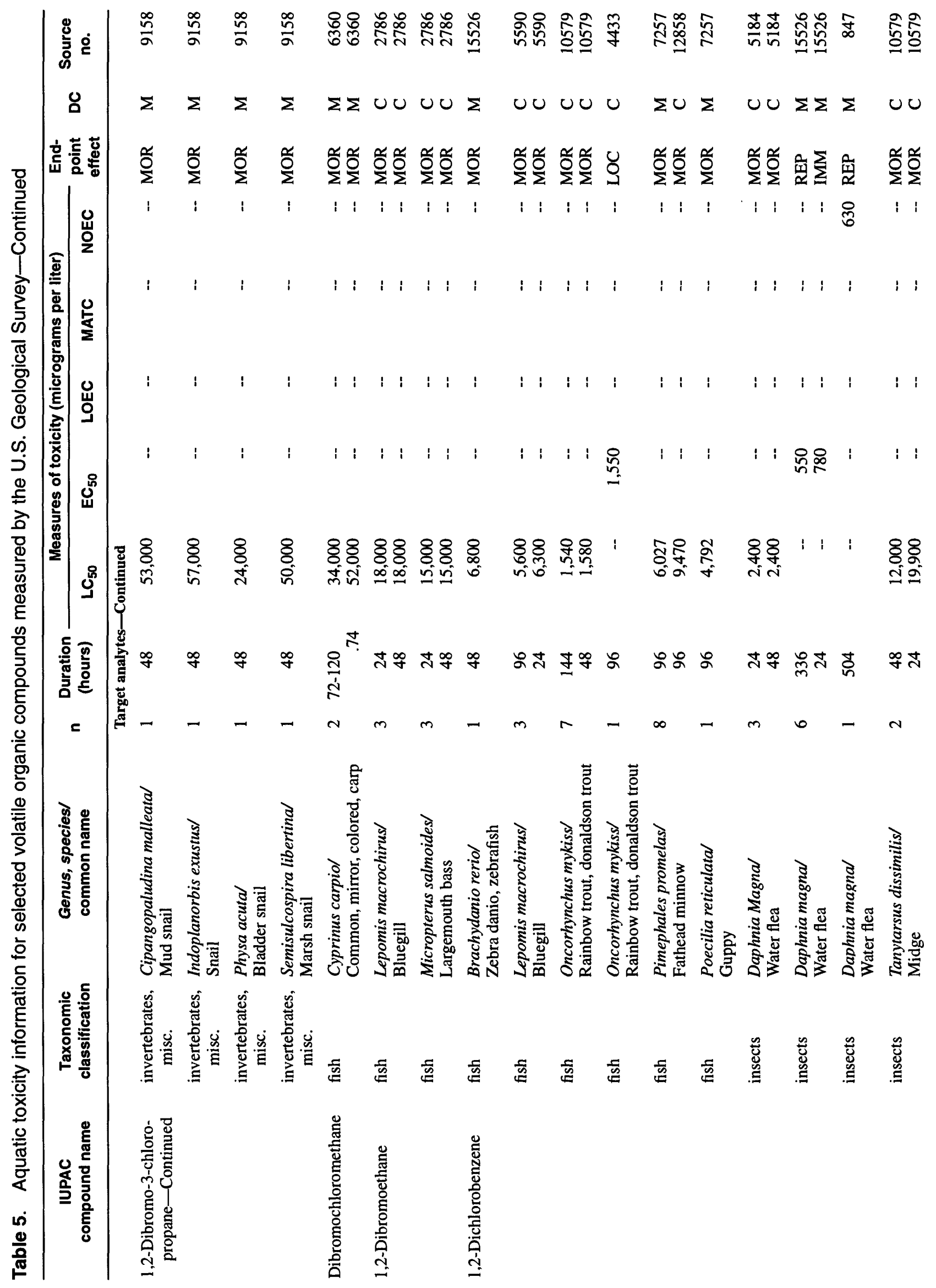




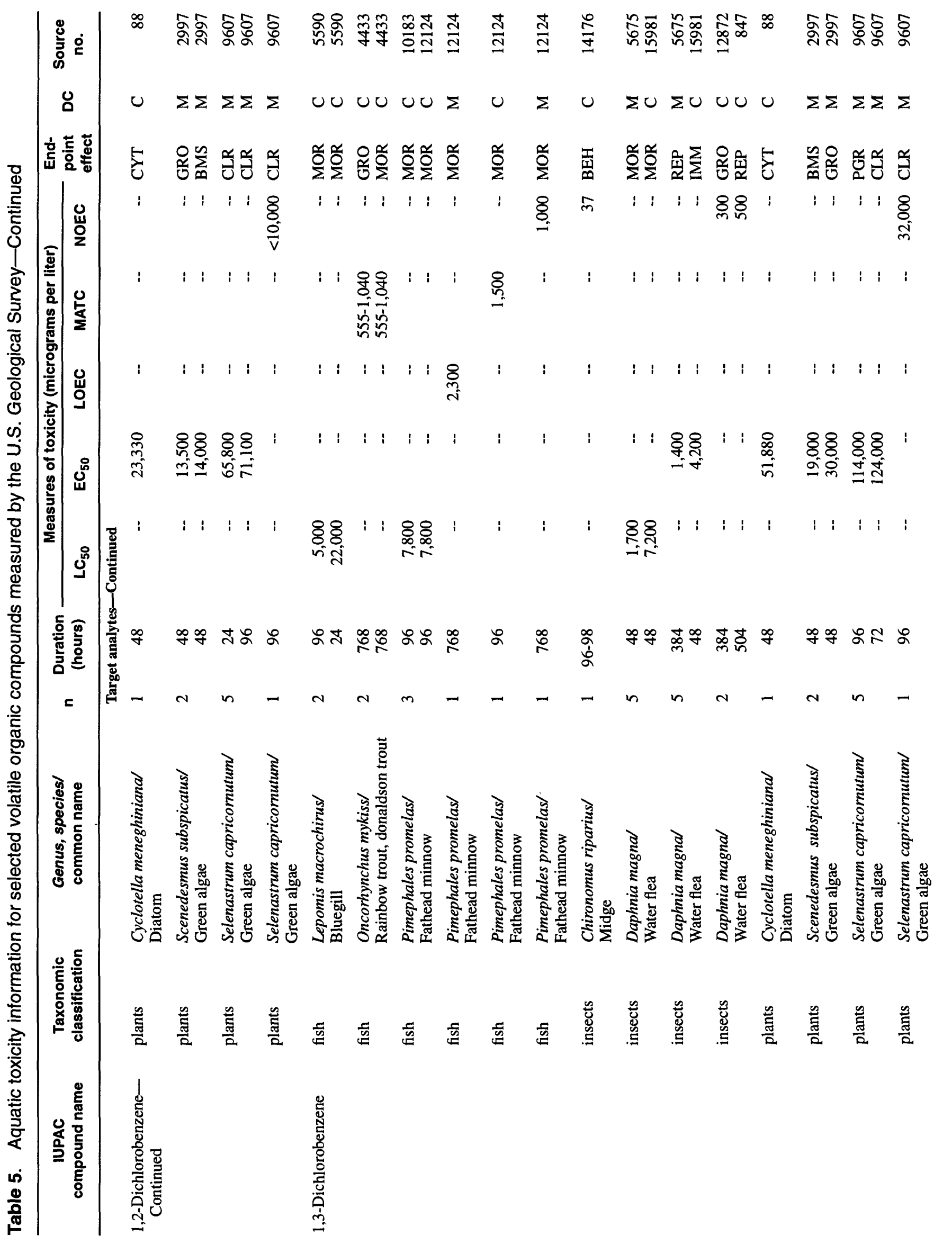




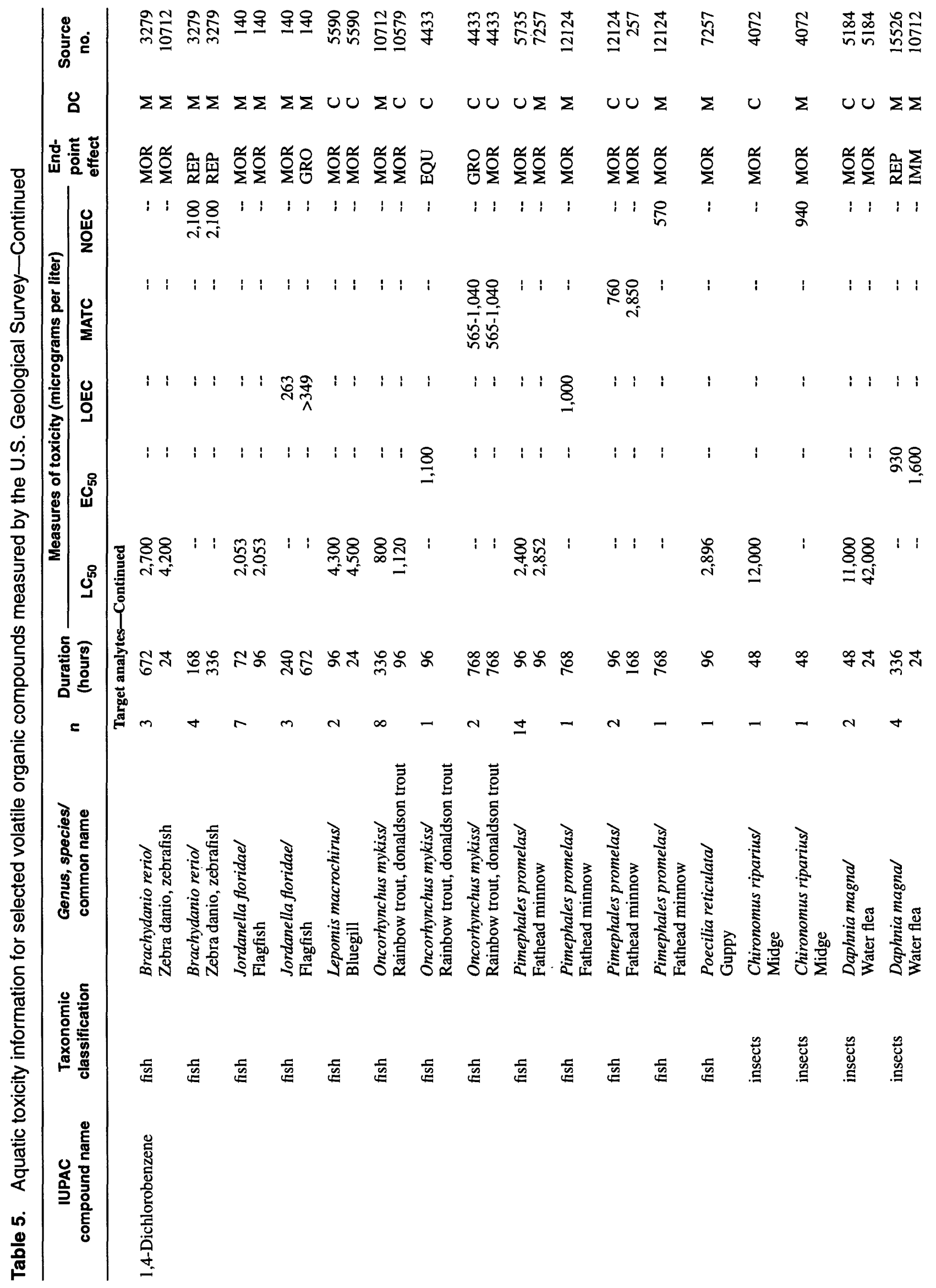




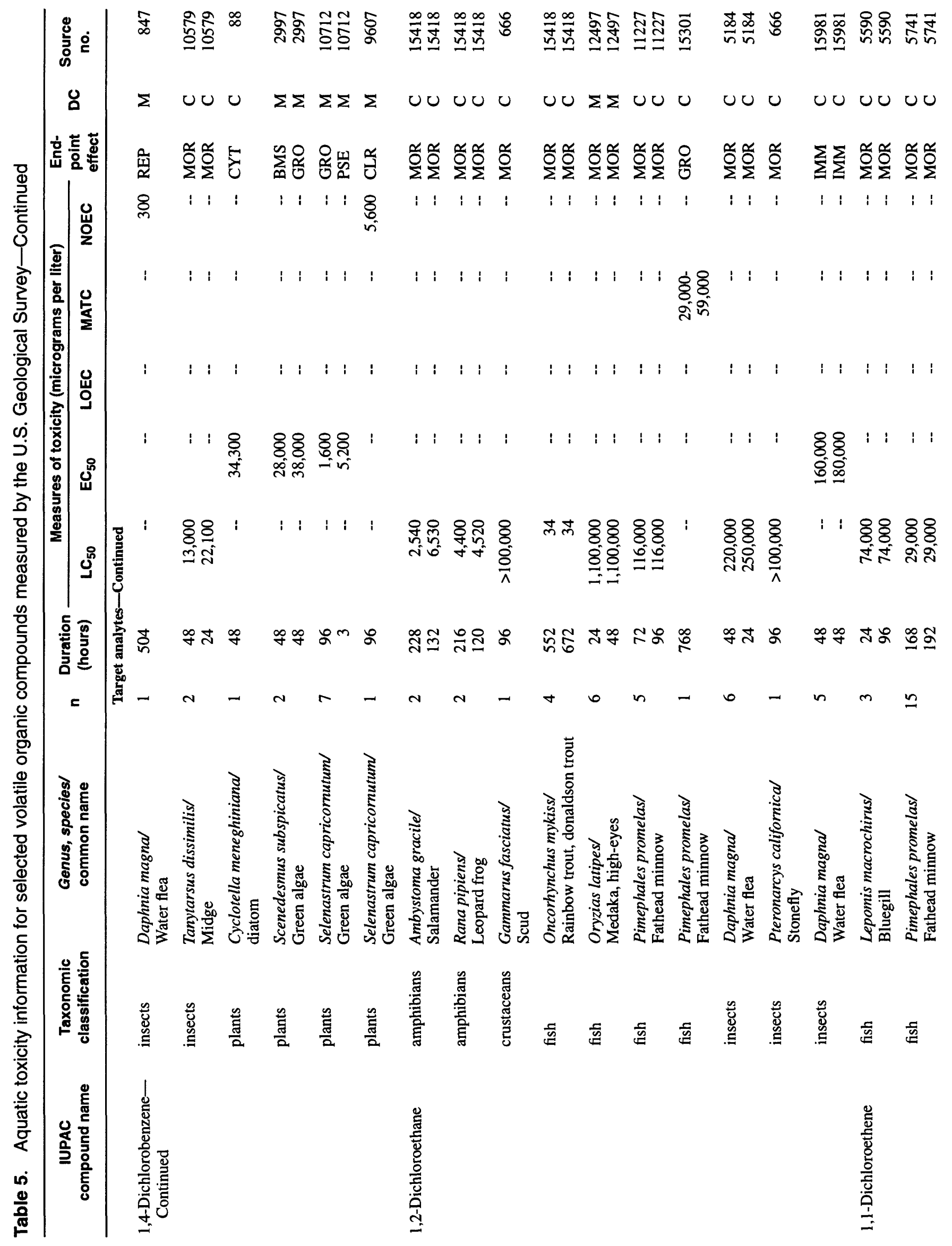




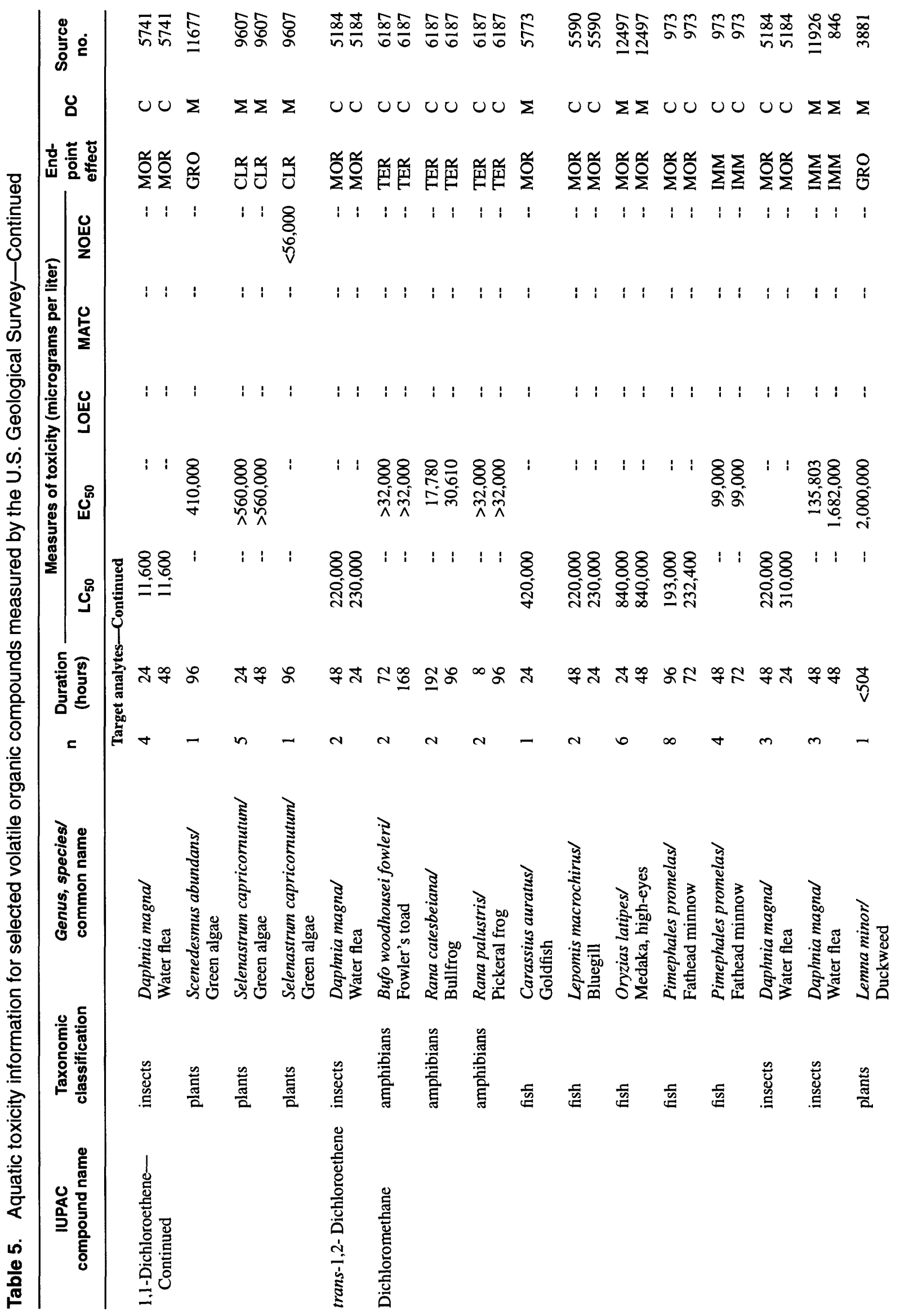




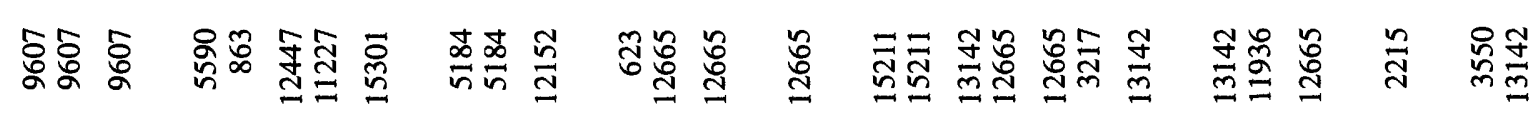
$\Sigma \Sigma \Sigma 0000000 \Sigma 0000 \Sigma \Sigma \Sigma 000 \Sigma \Sigma 00 \Sigma \Sigma \Sigma$

: : : :

1
88
80
80
000

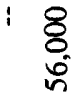

i i 1

$=$

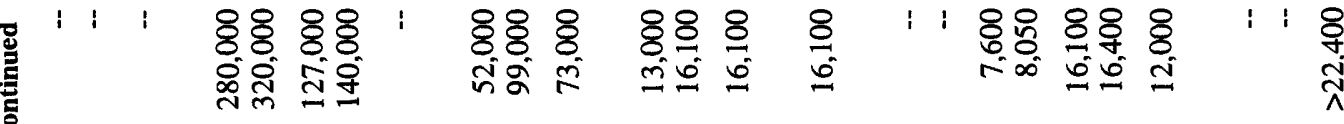

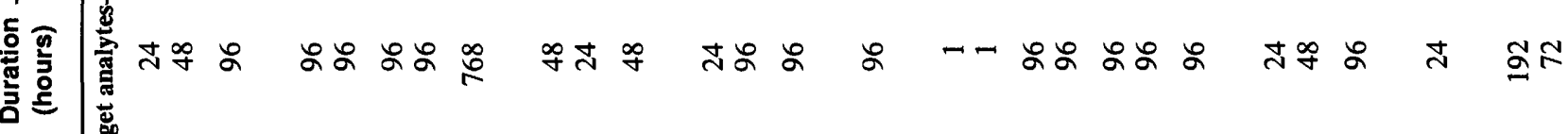
焉
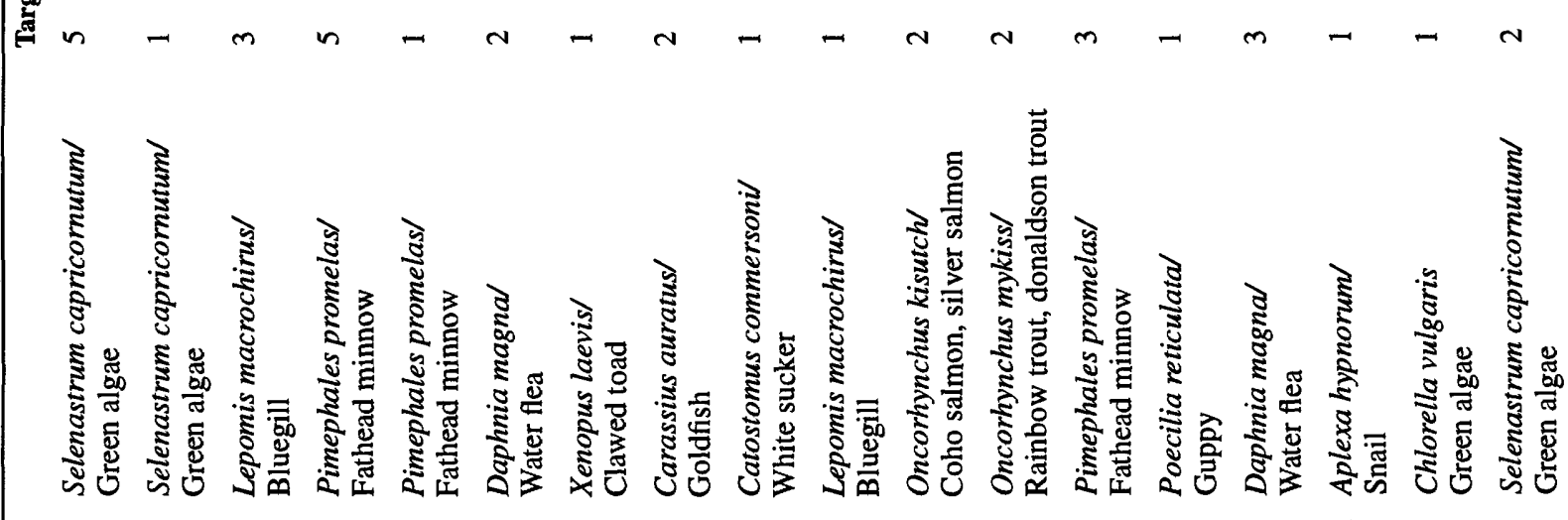

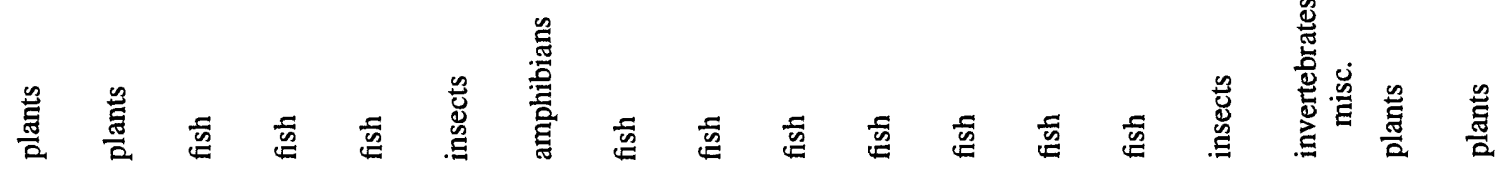




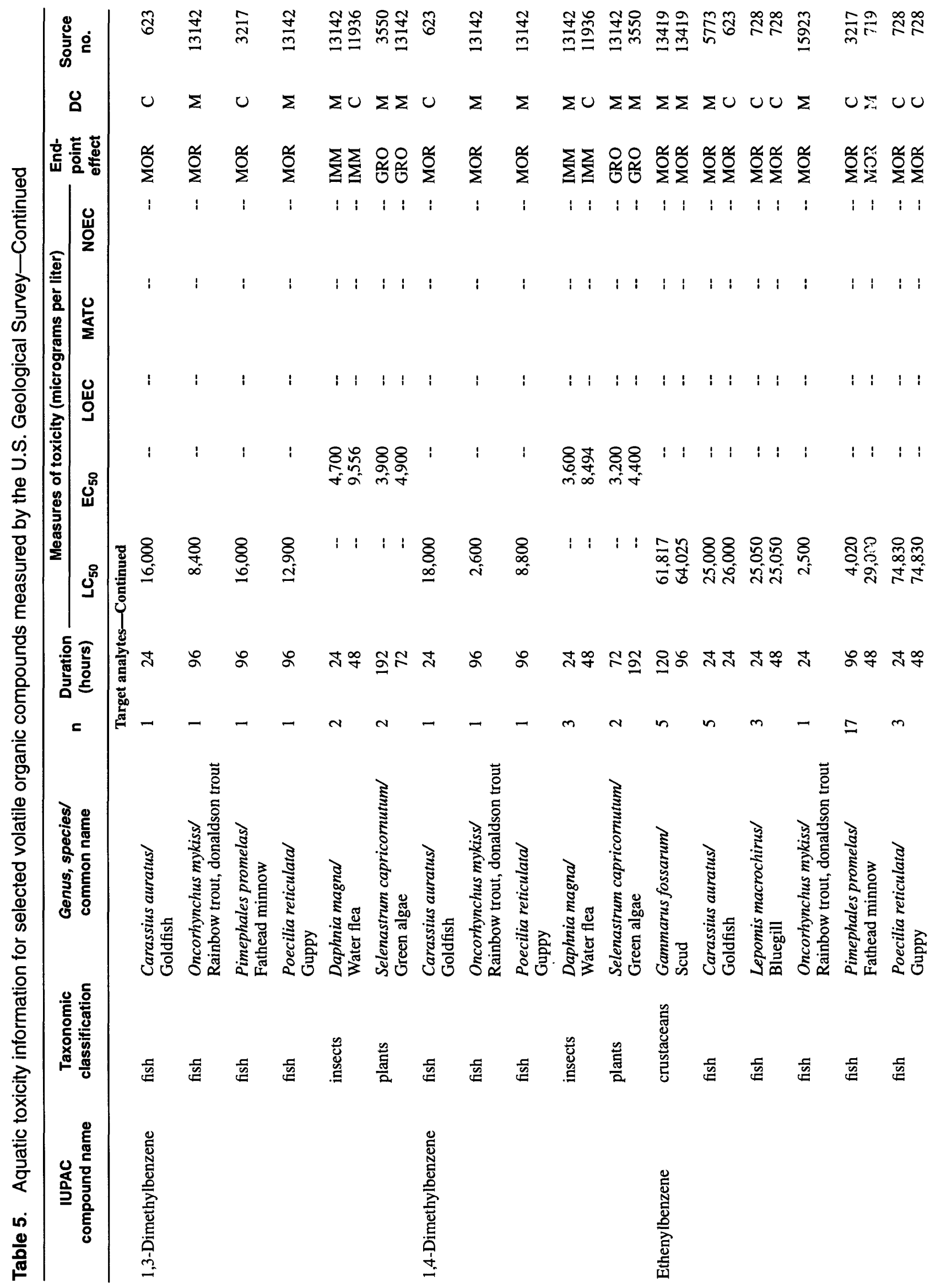




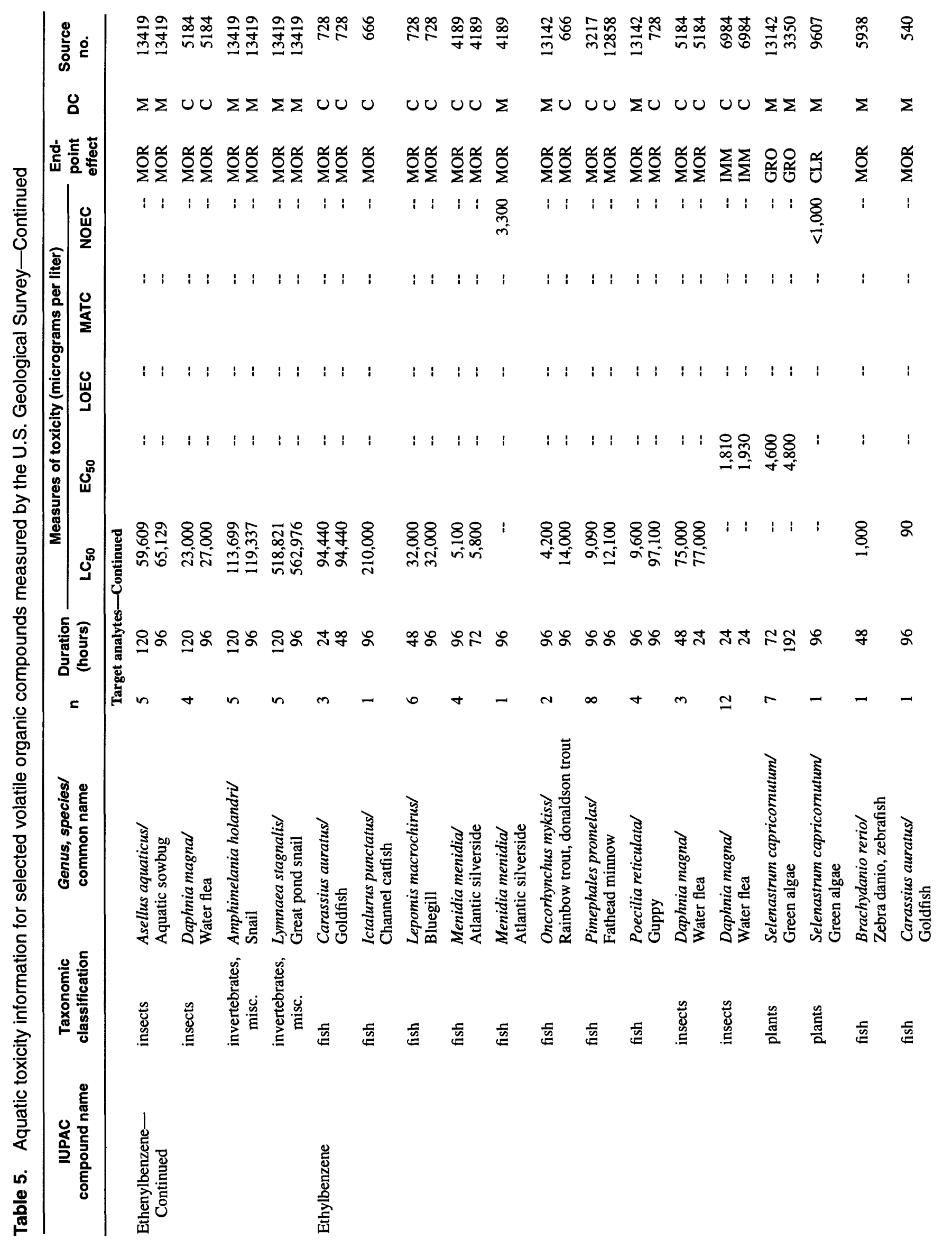




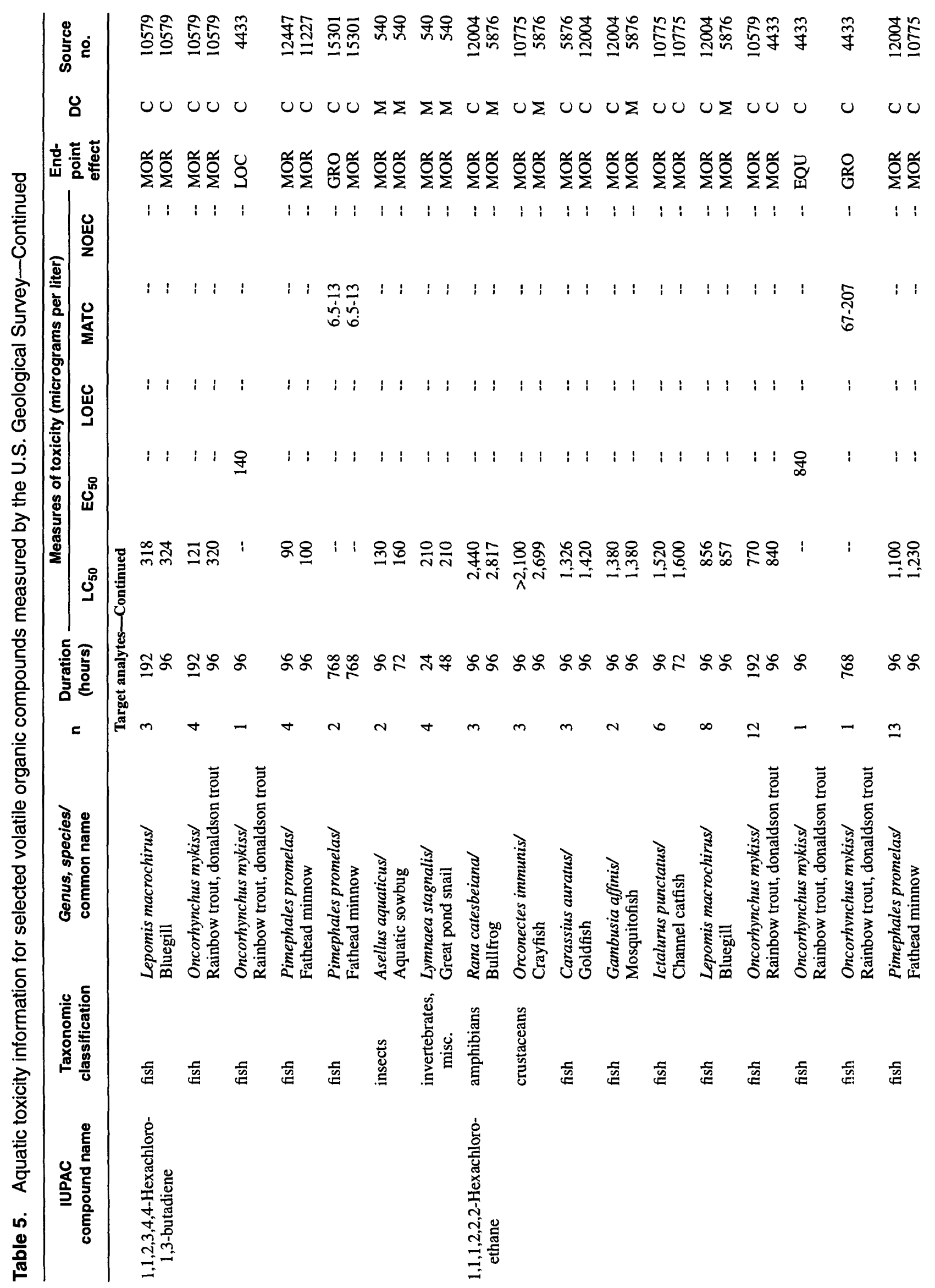




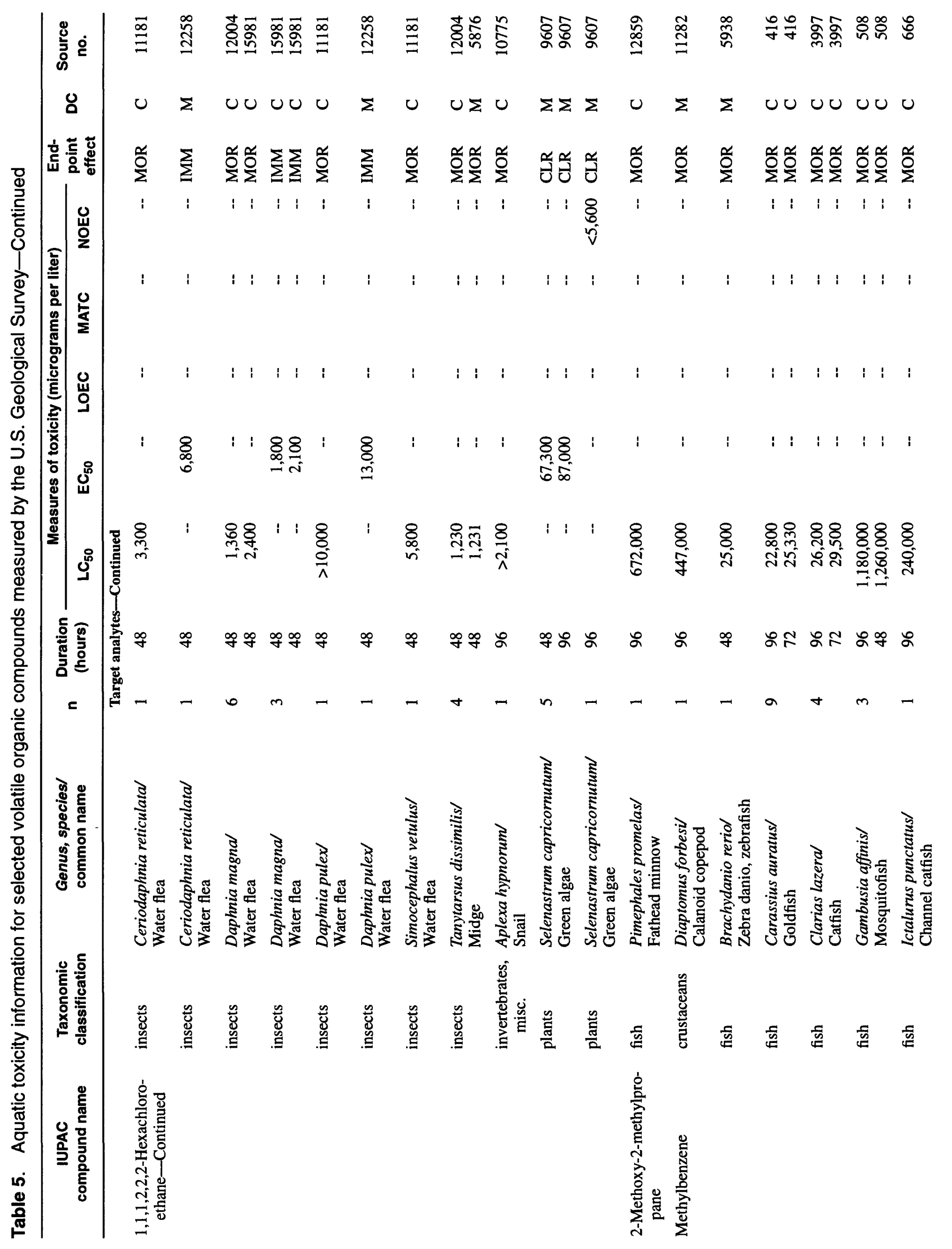




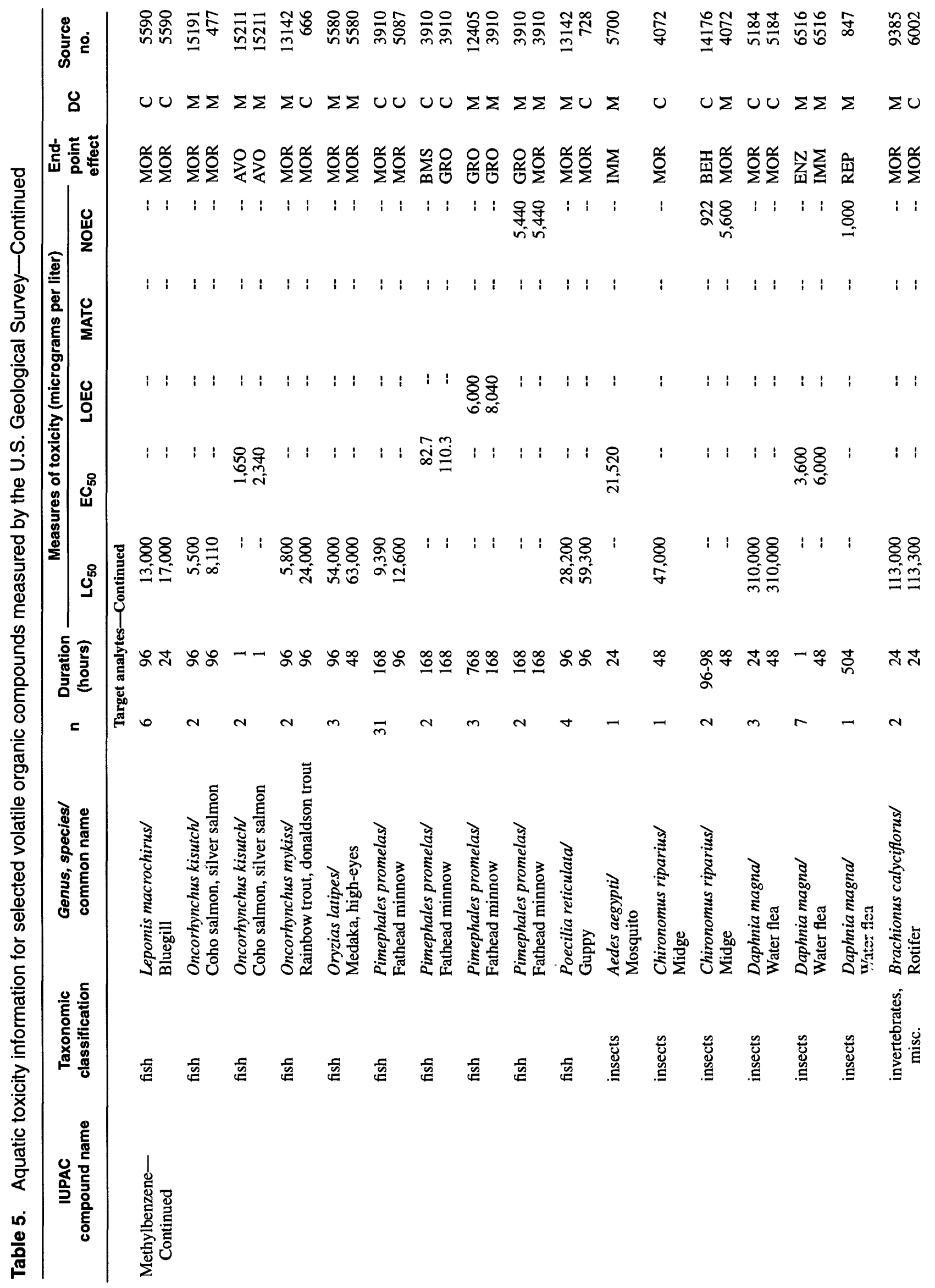




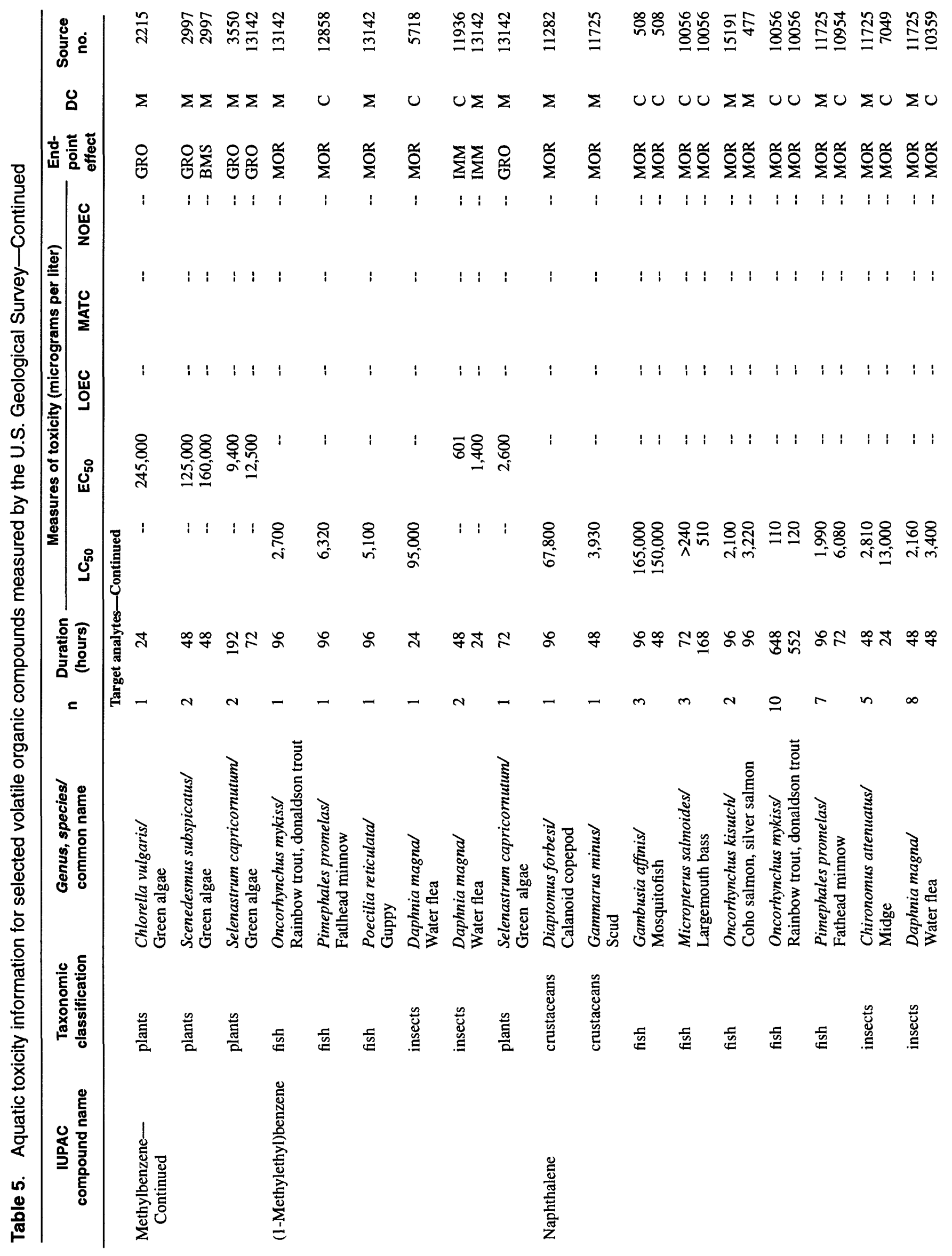




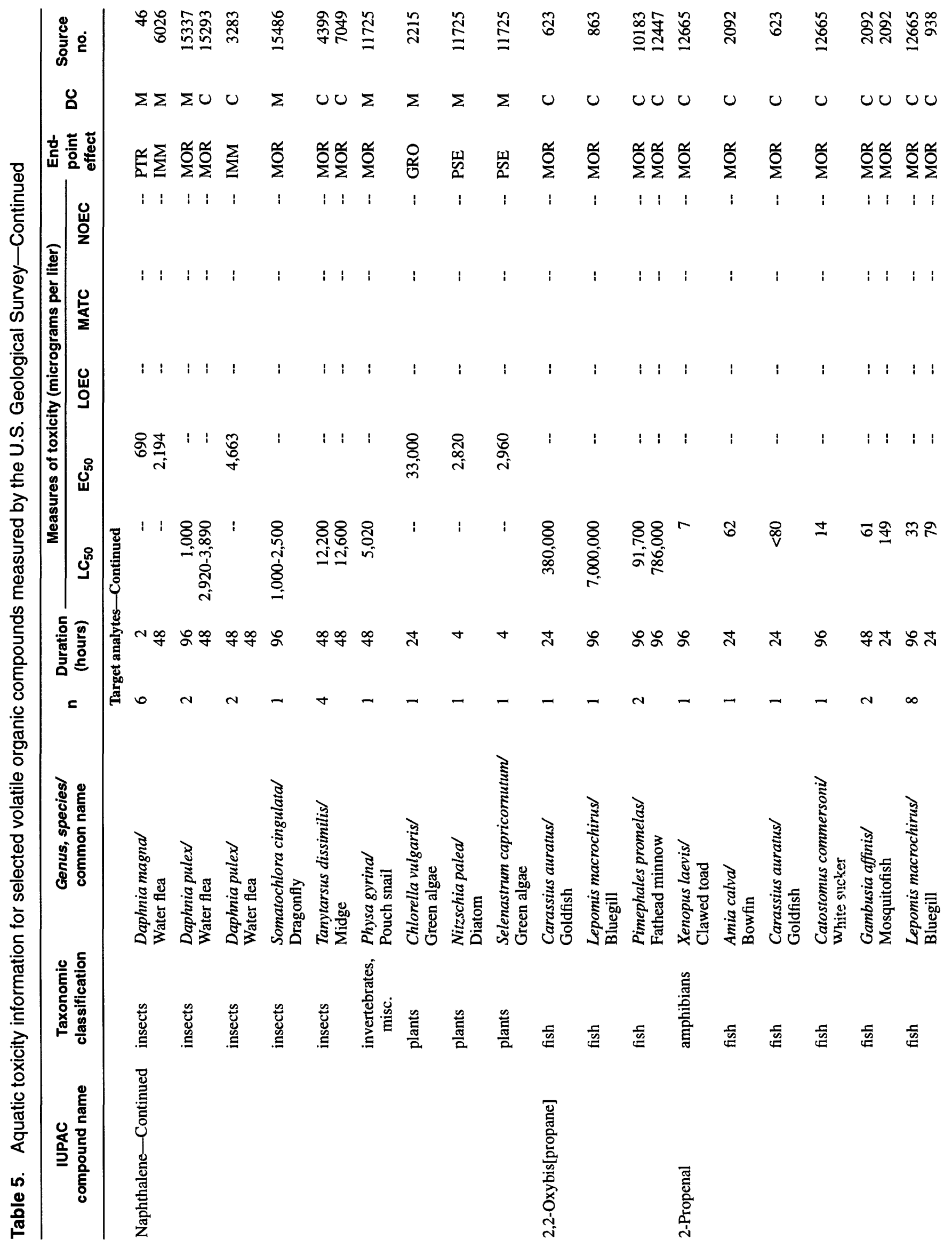




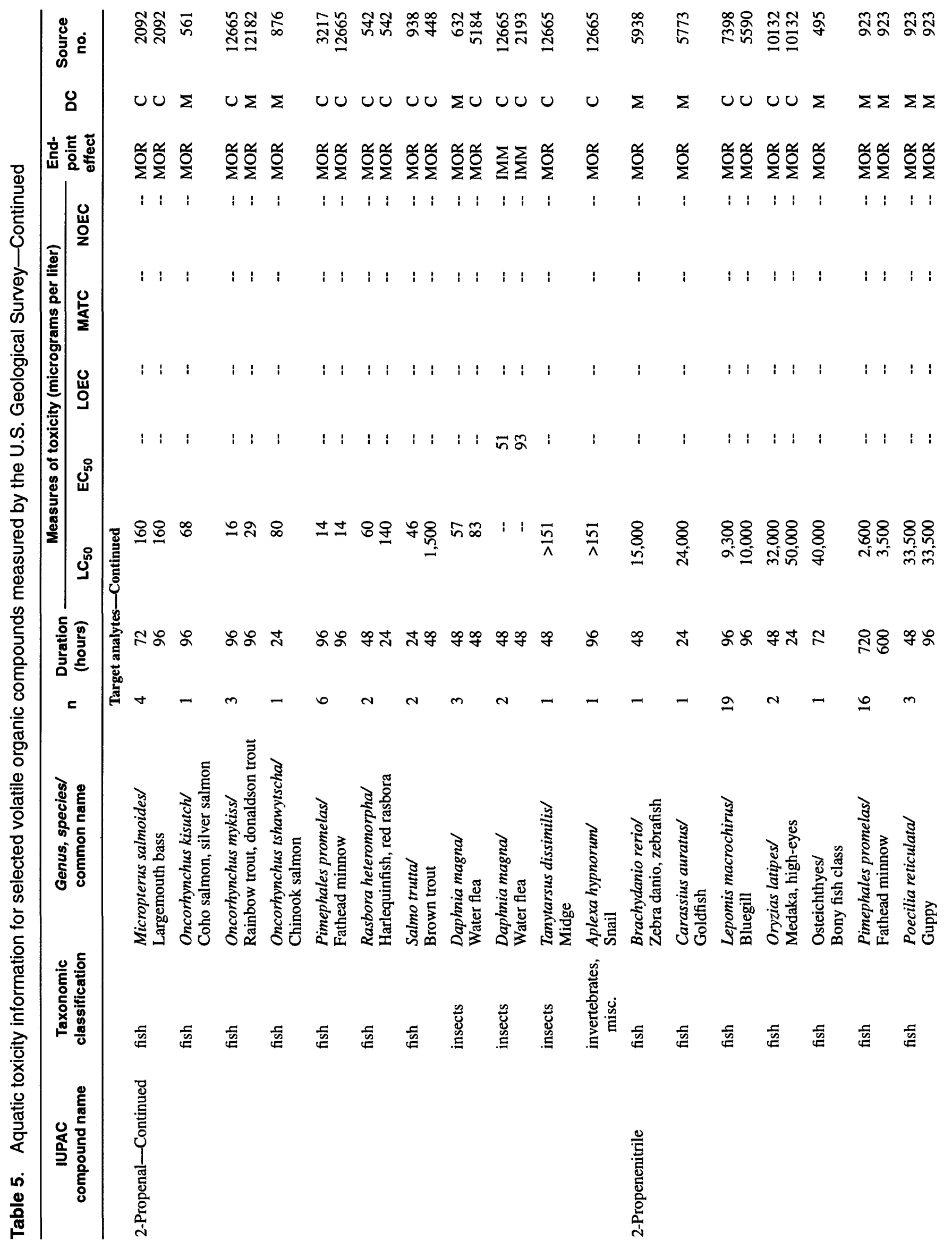




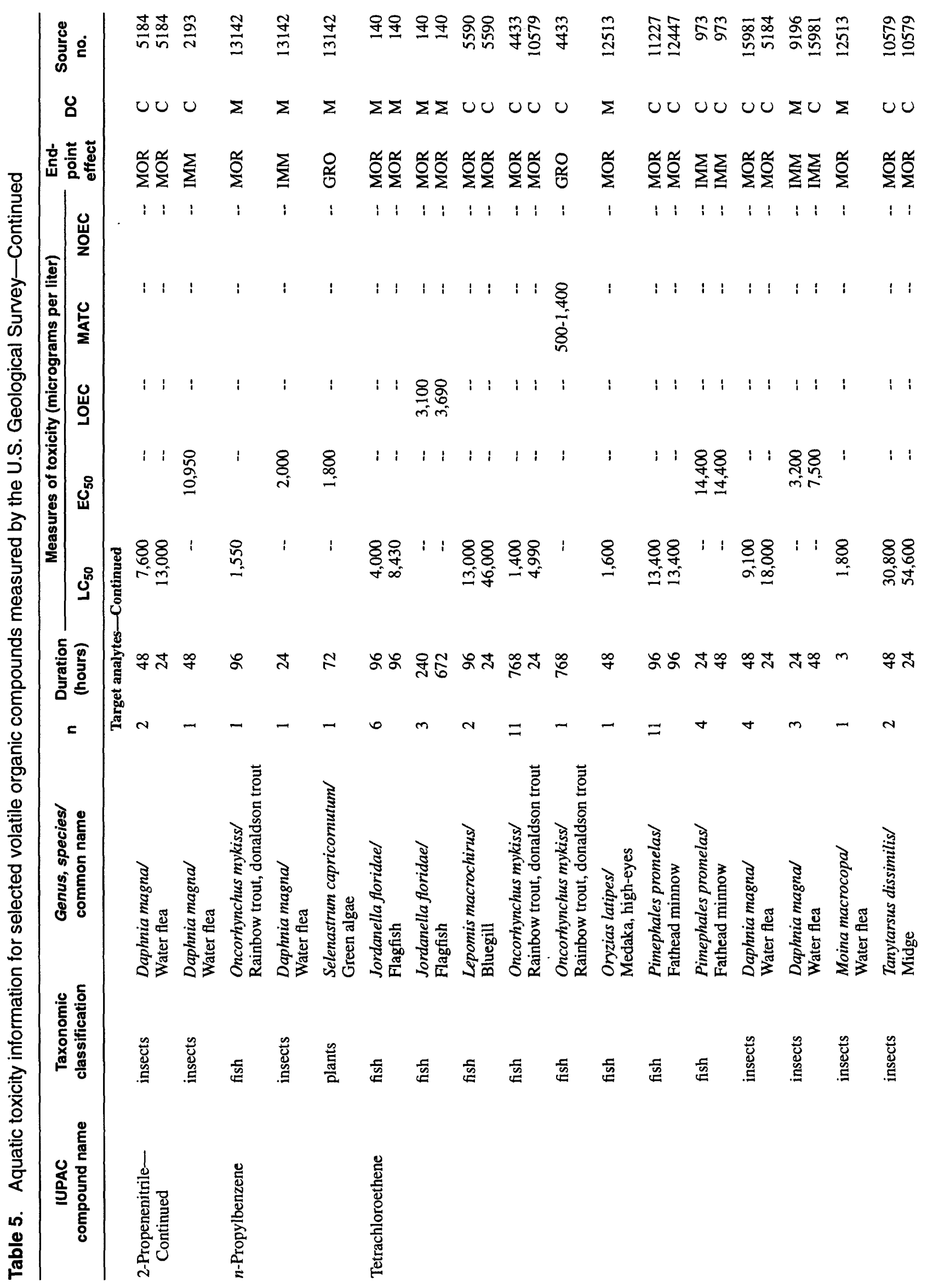




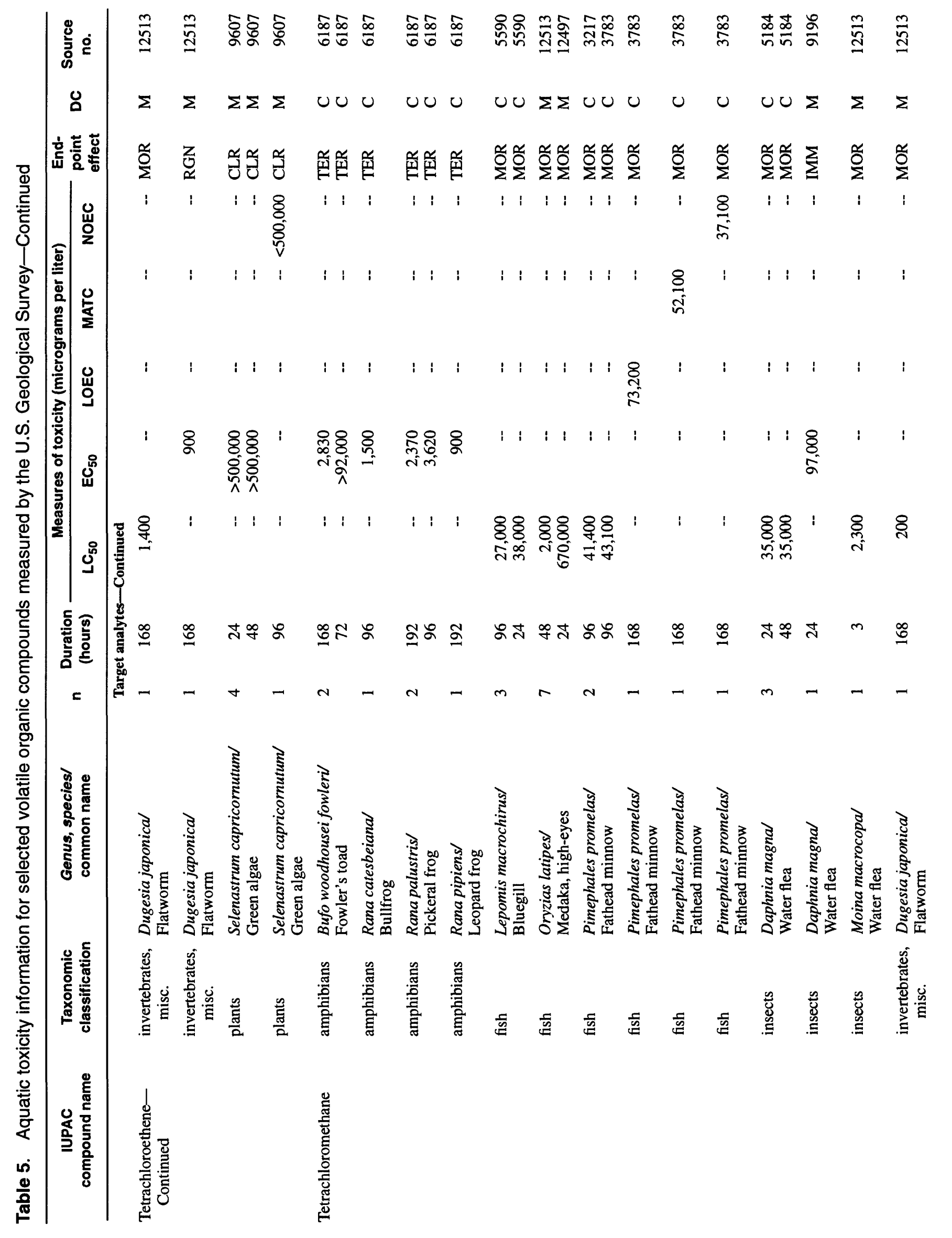




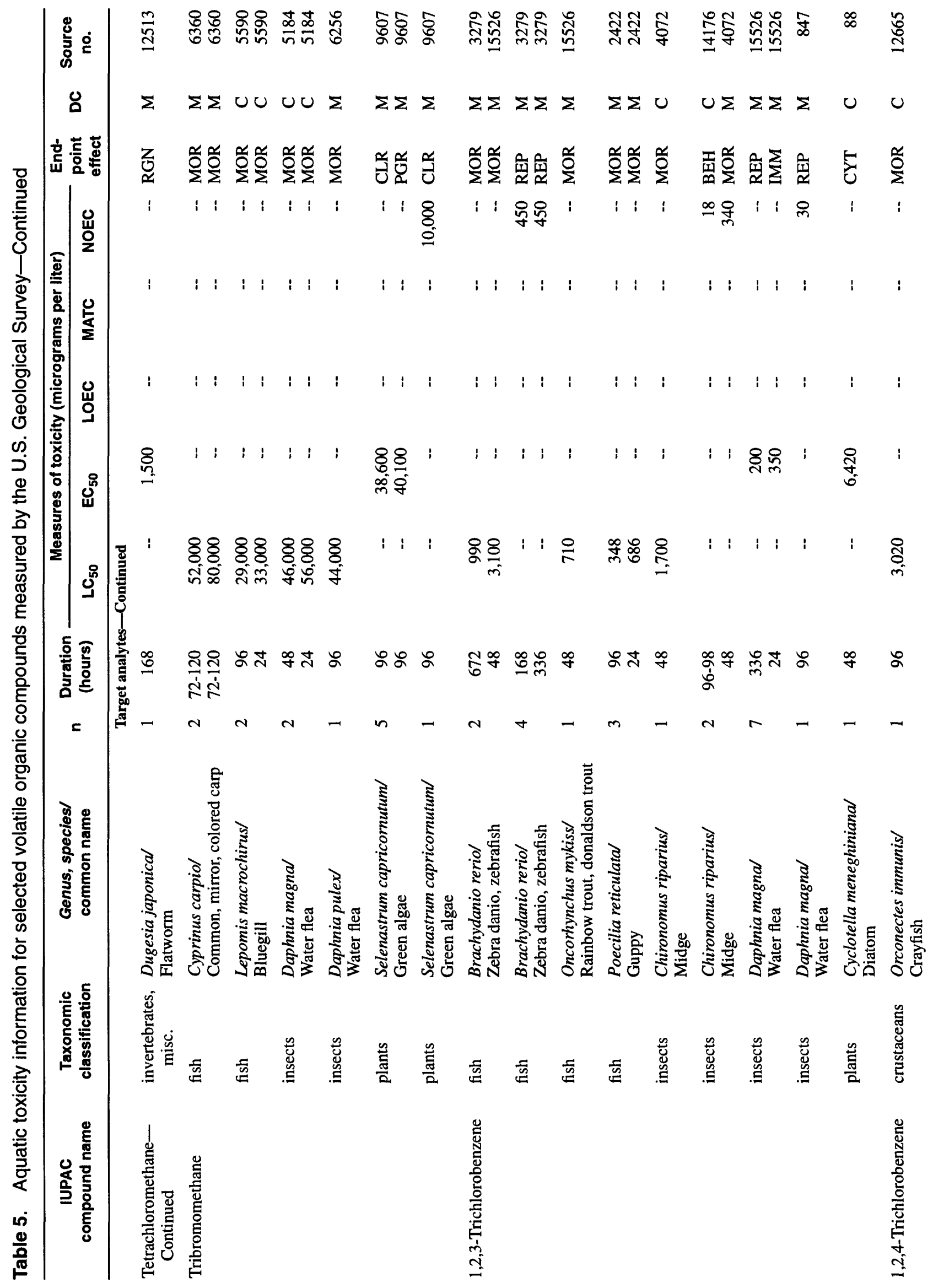




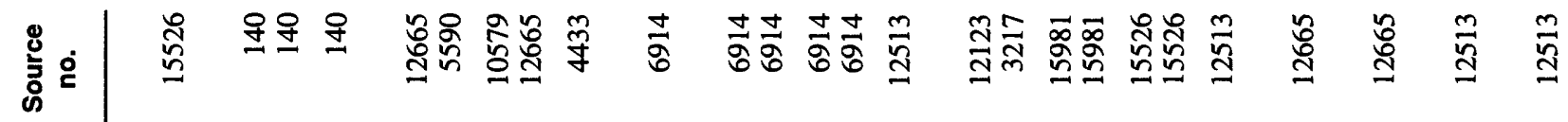

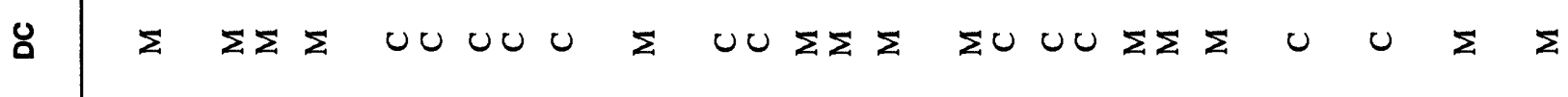

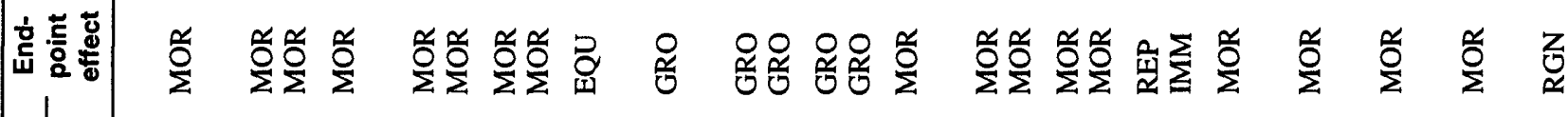

品

! $\quad$ 1

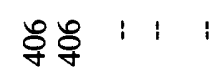

i 곡

윰요

$\stackrel{8}{2}$

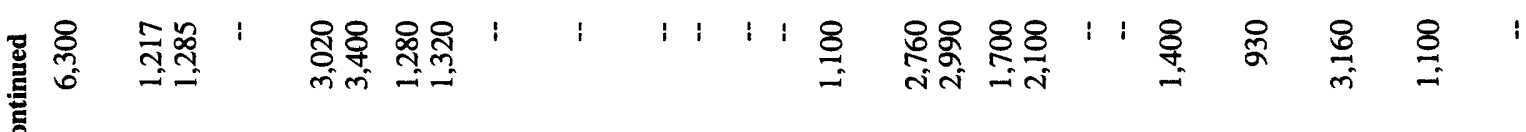

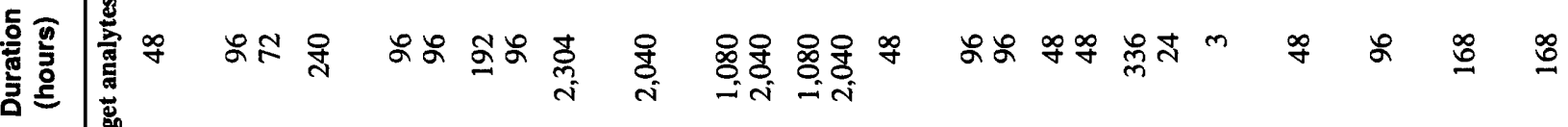

$0 \rightarrow m$

$\frac{\substack{0 \\ .0}}{\frac{0}{0}}$

10

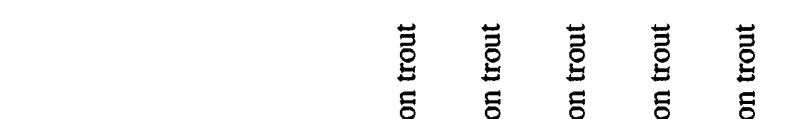

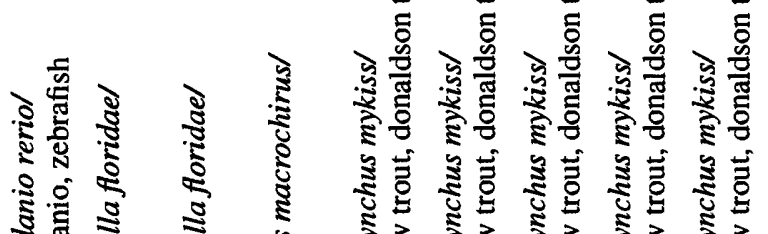

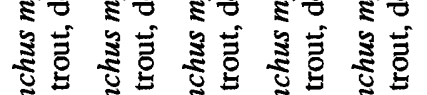

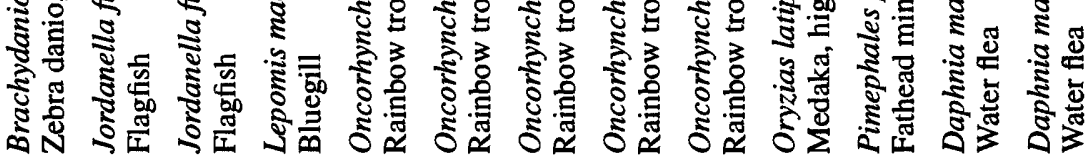

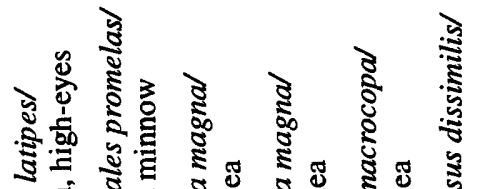

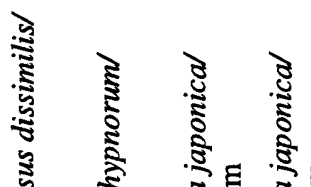




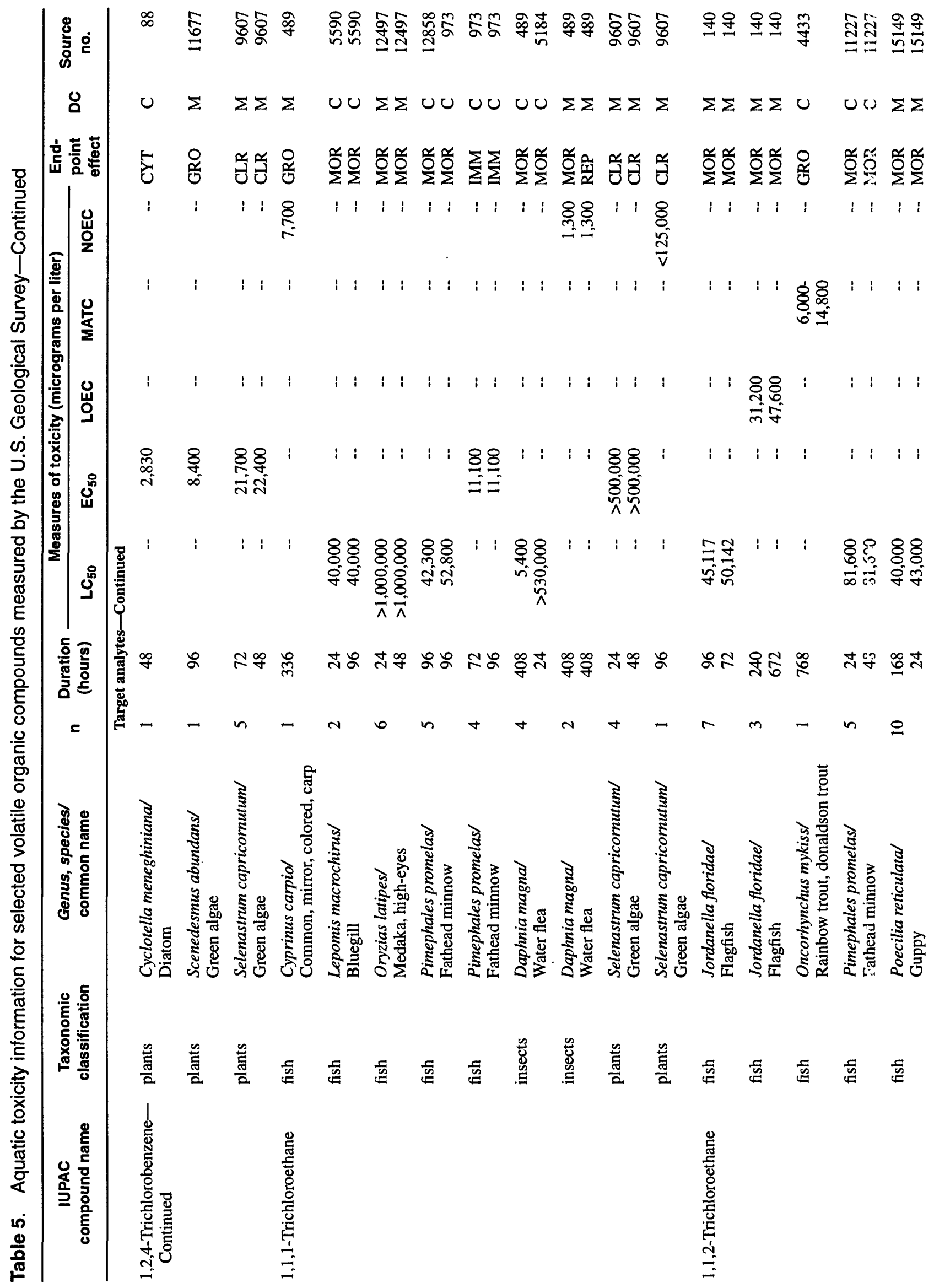




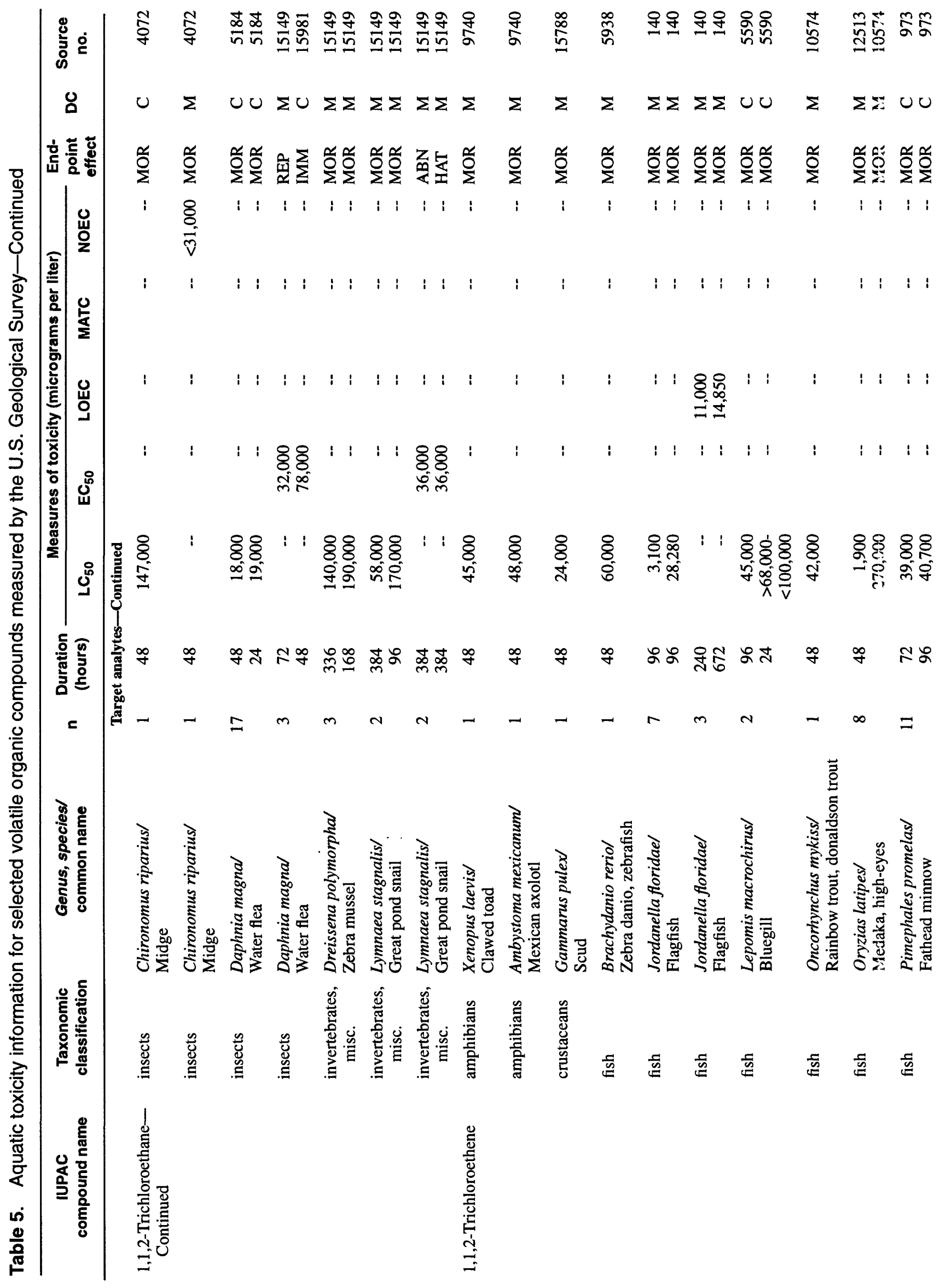




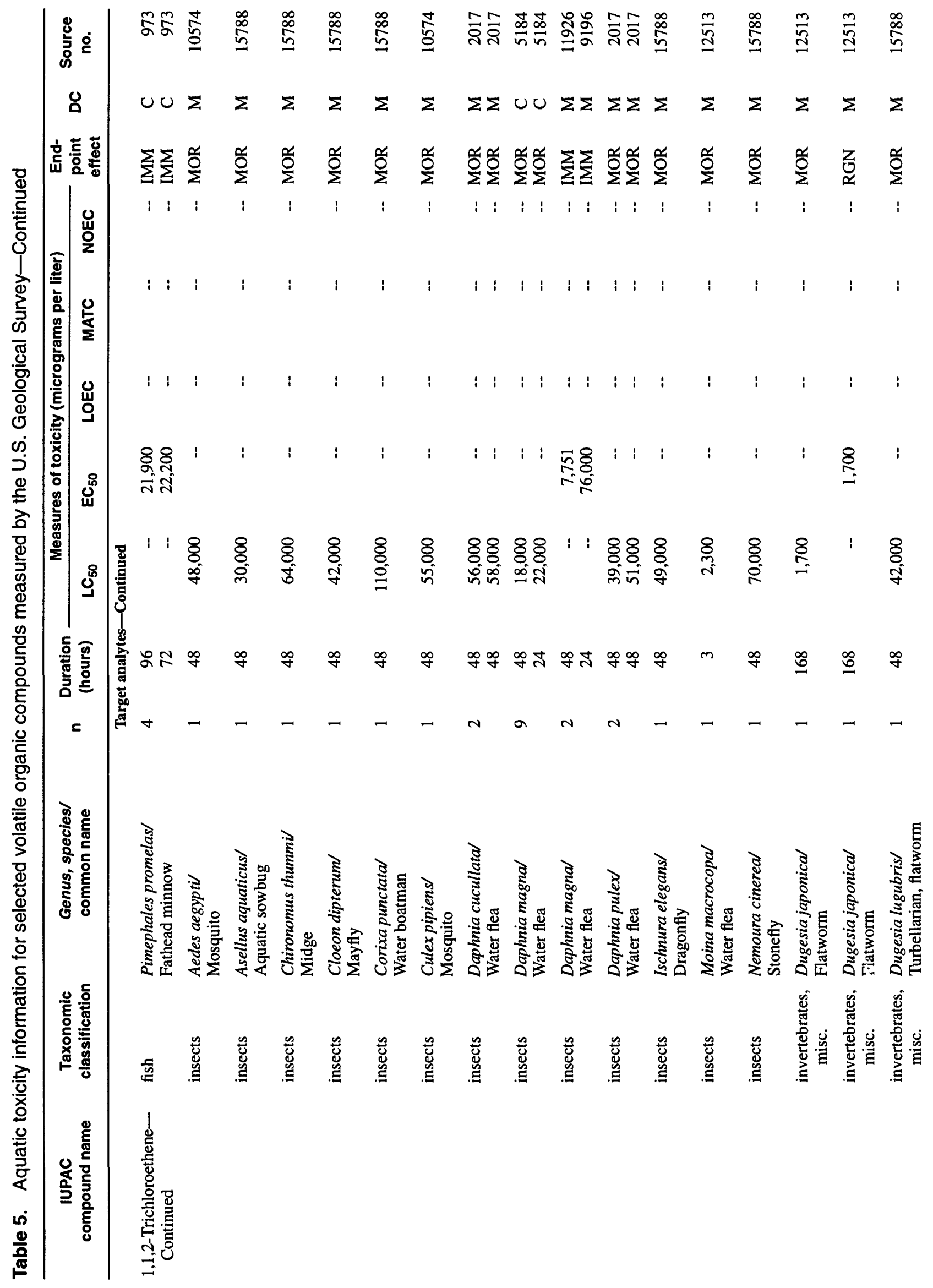




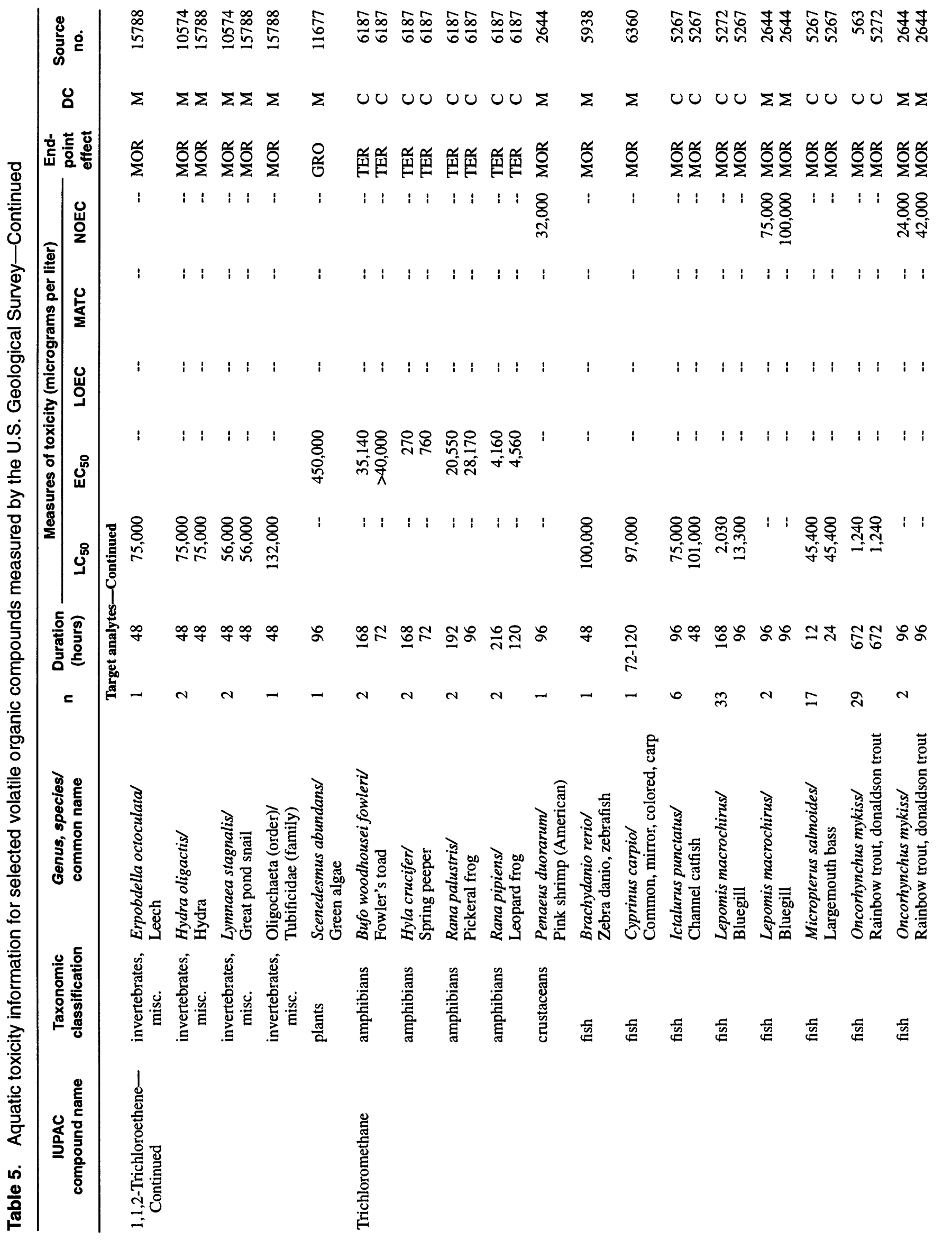




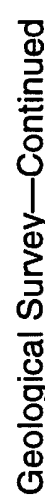

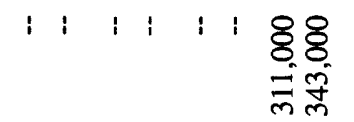

ᄃ ศึ

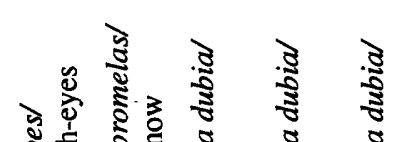

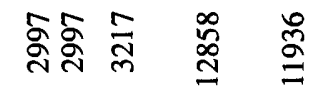
$\Sigma \Sigma 0$

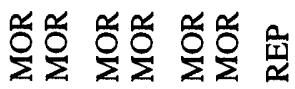

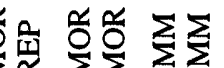

용

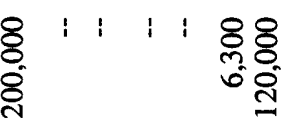

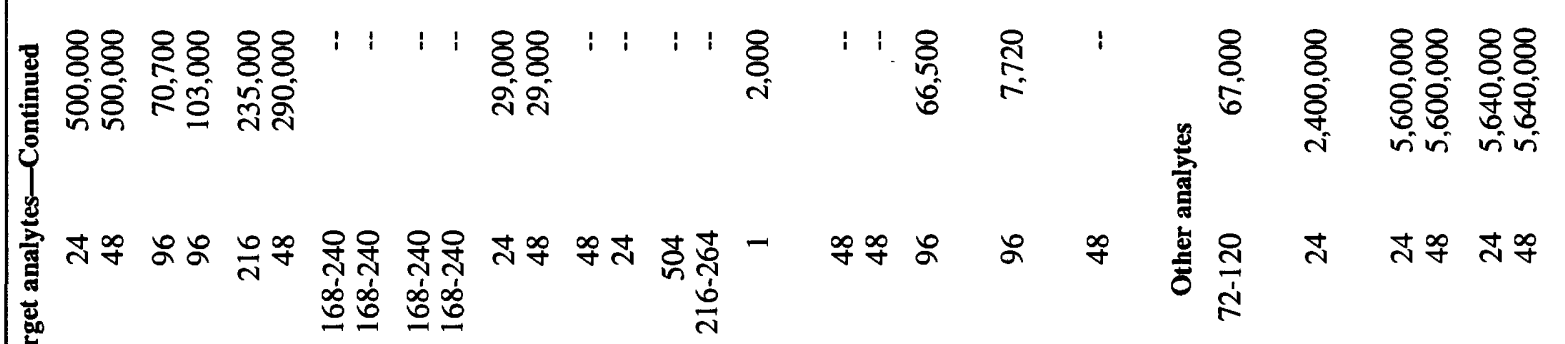

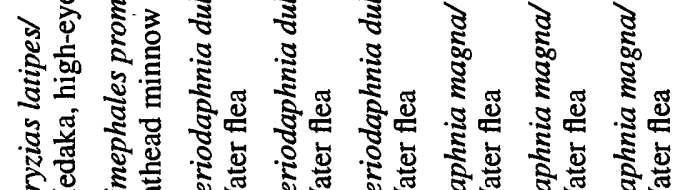

\section{ริ}

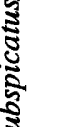

ฐूँ<smiles>CCC1CCC1</smiles>
范

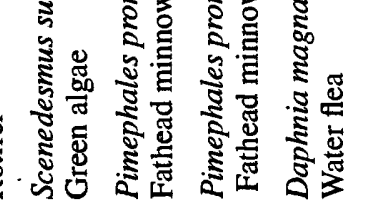

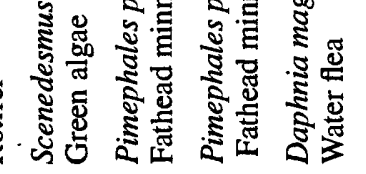

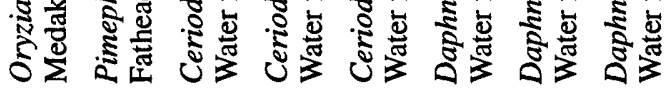

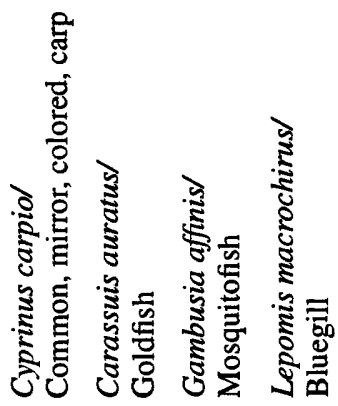

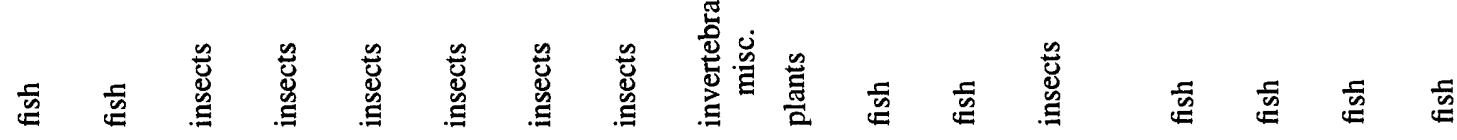




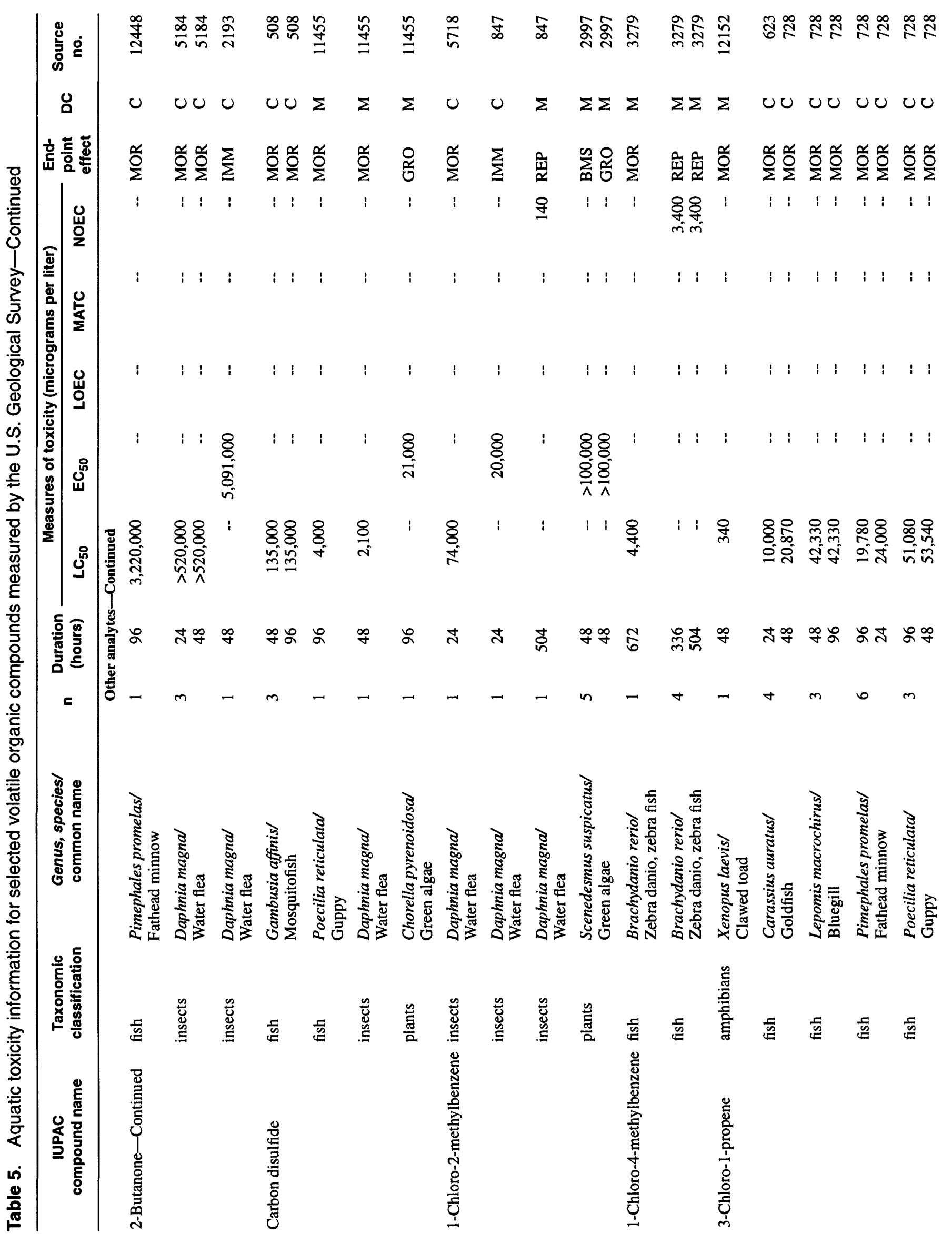




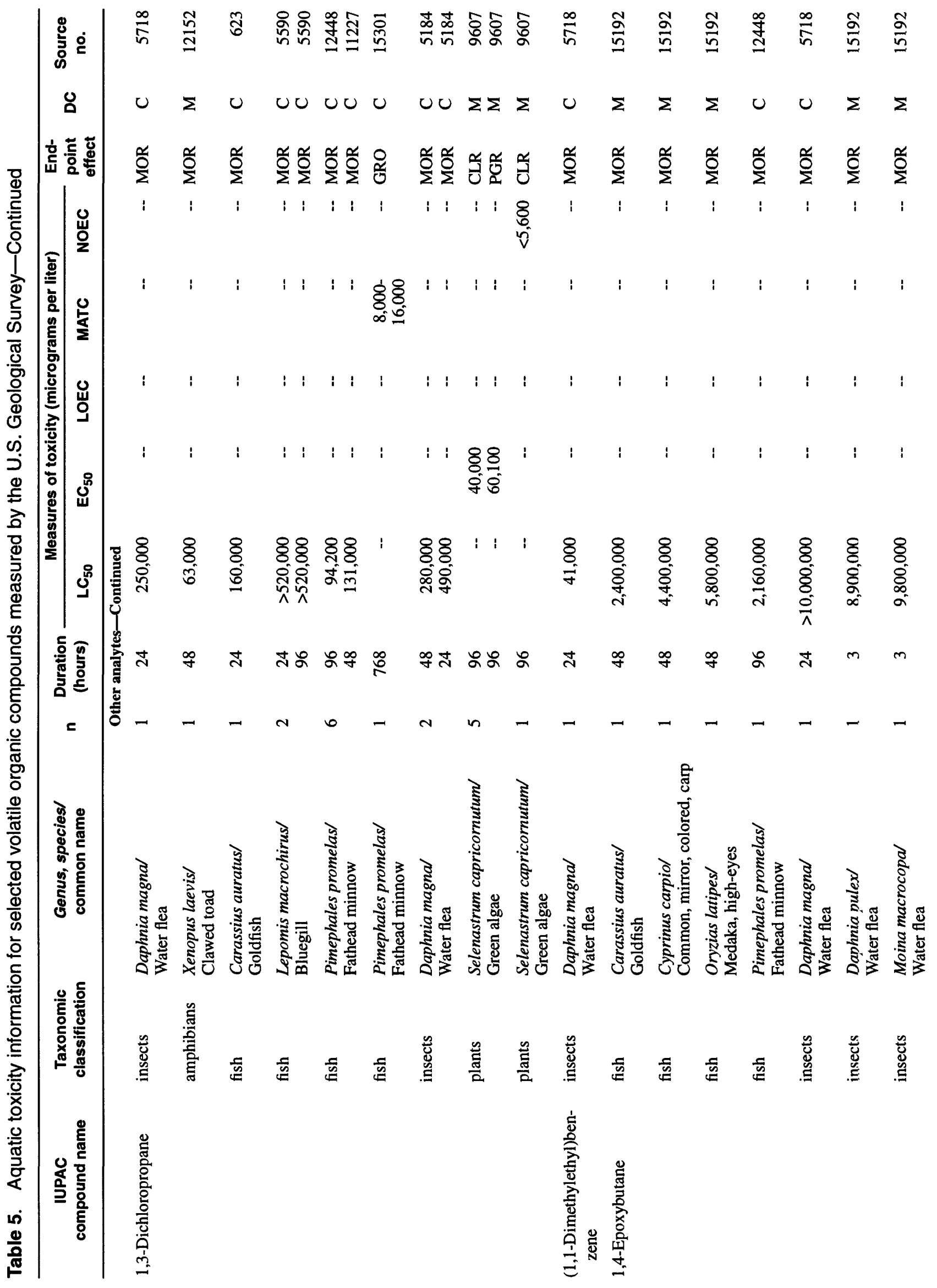

44 Summary of Published Aquatic Toxicity Information and Water-Quality Criteria for Selected VOCs 


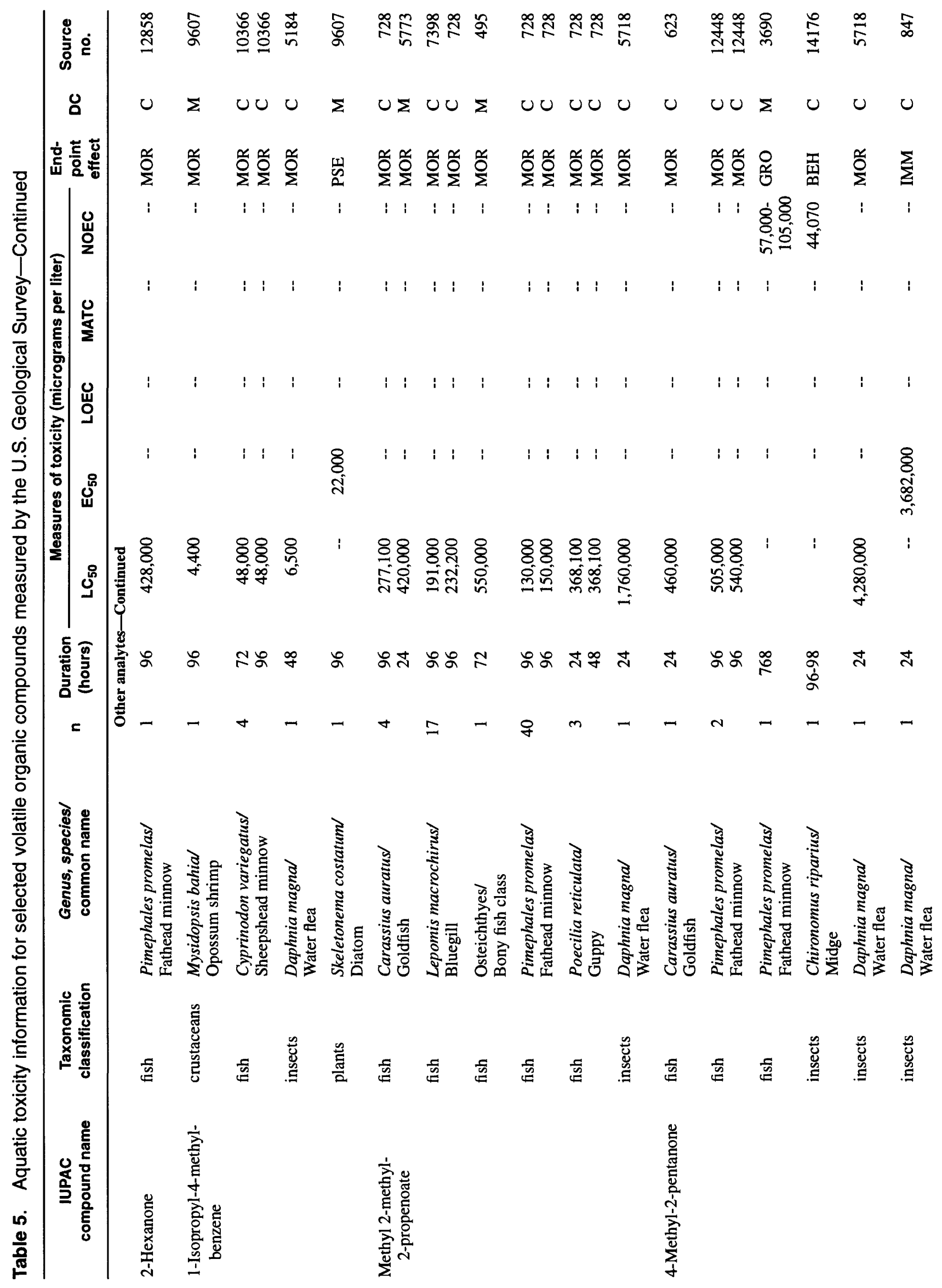




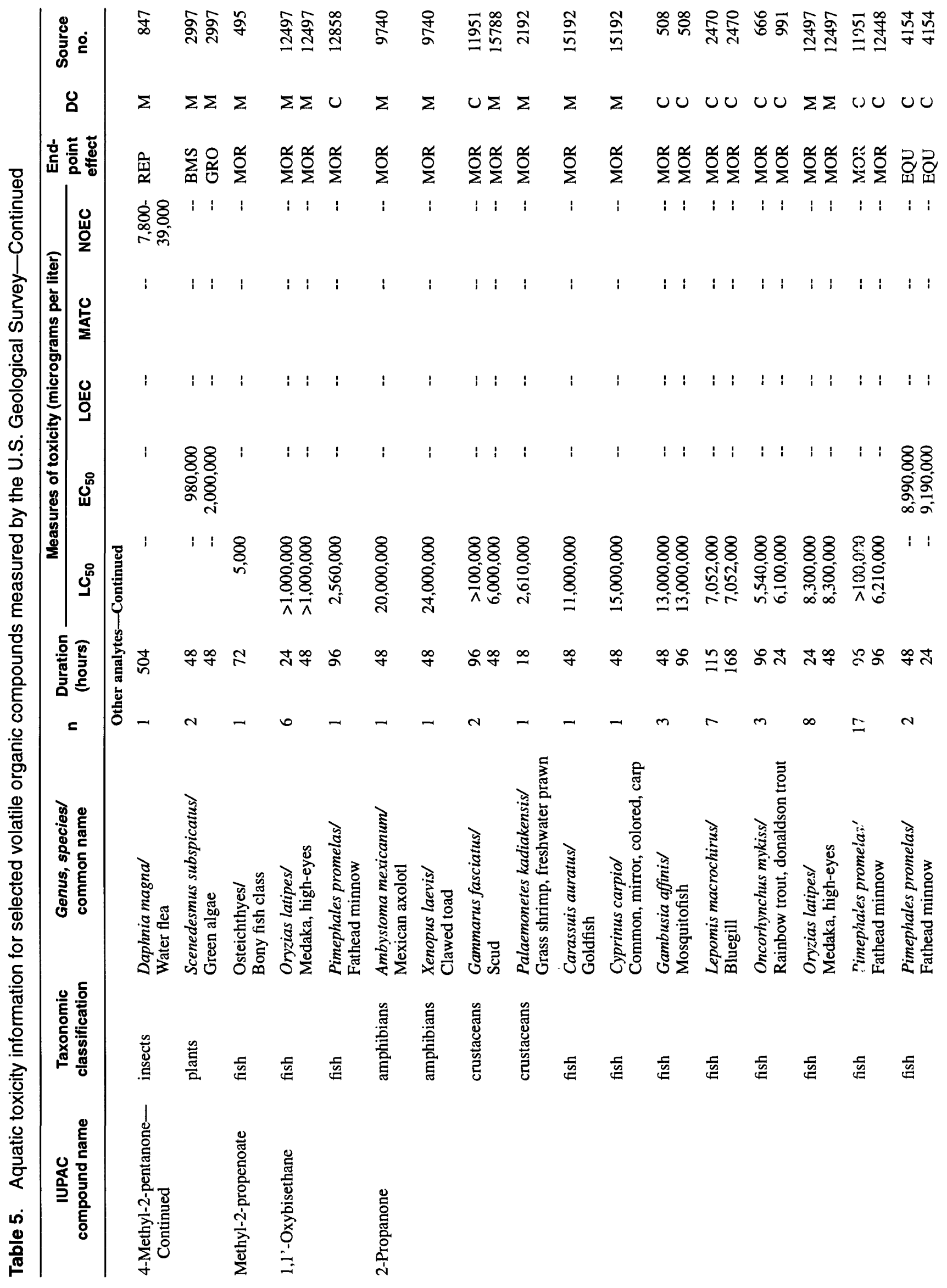




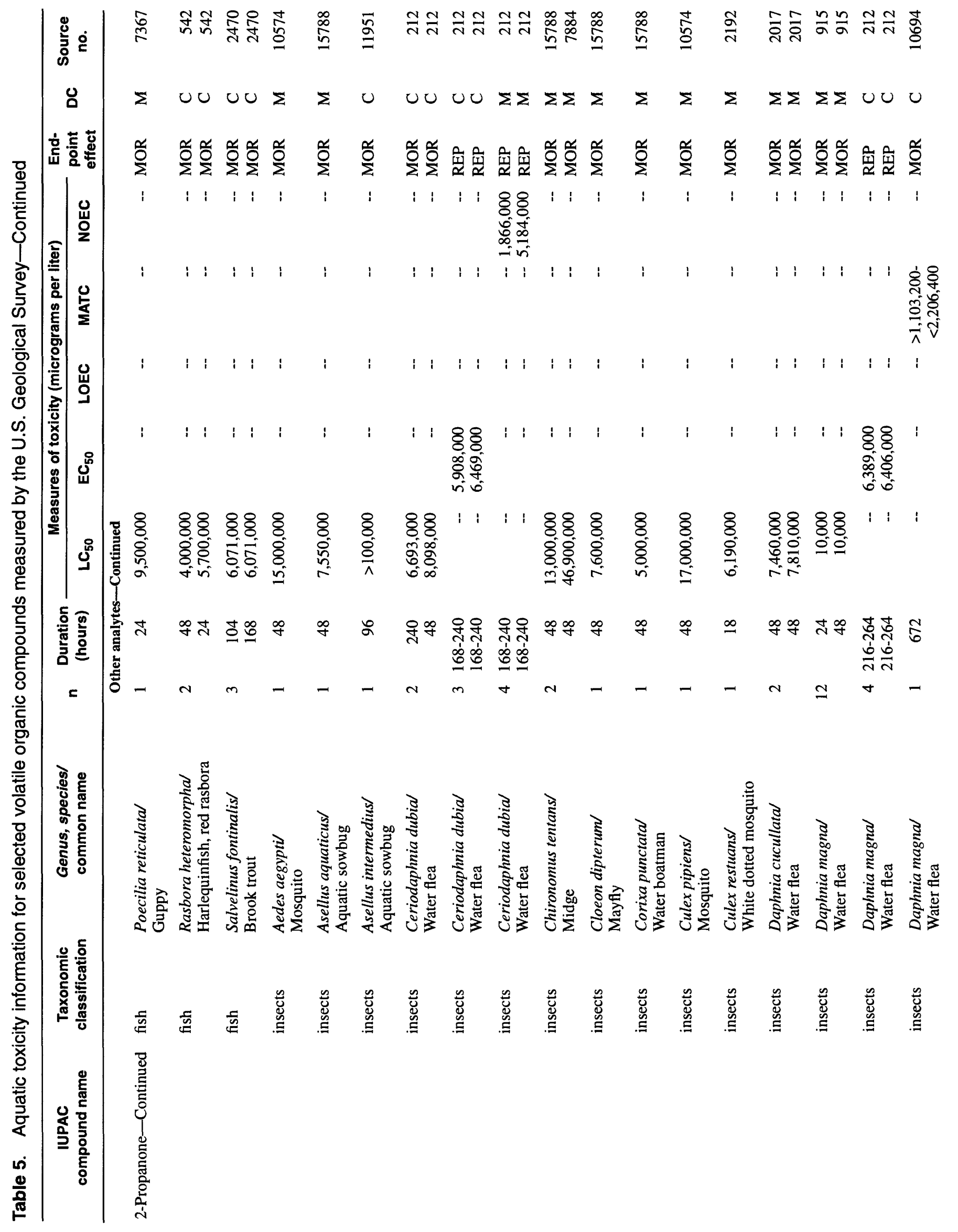




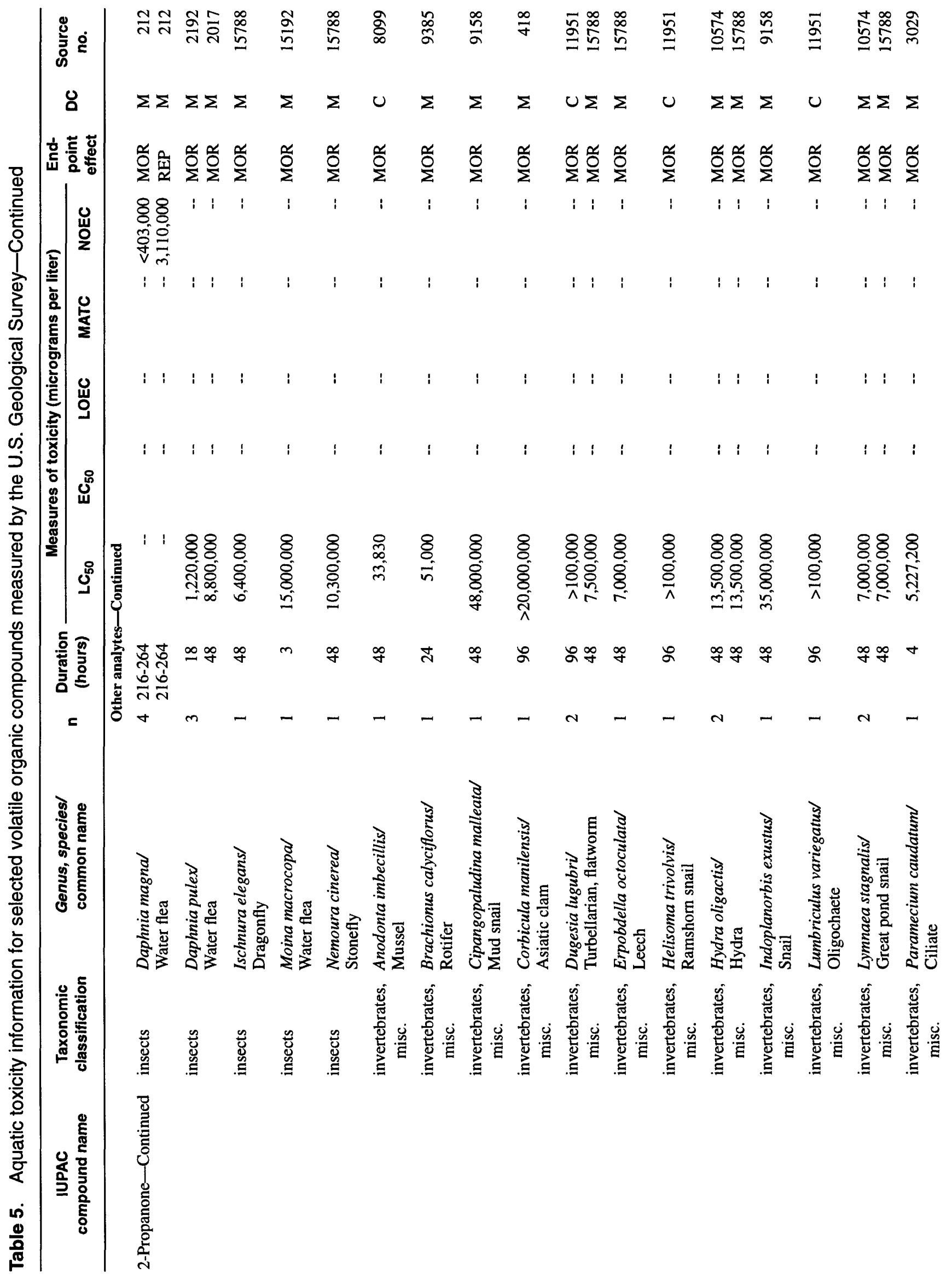




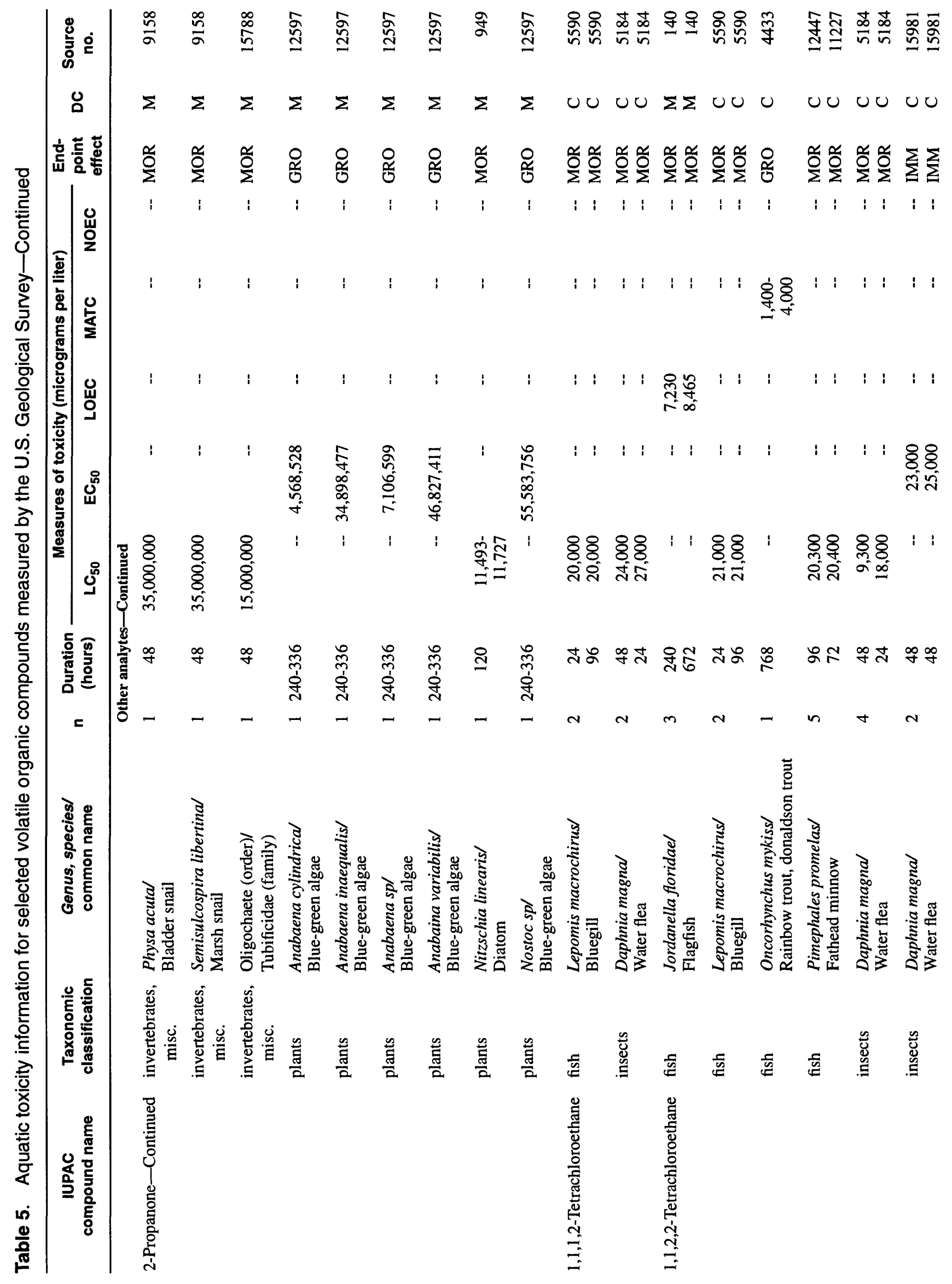




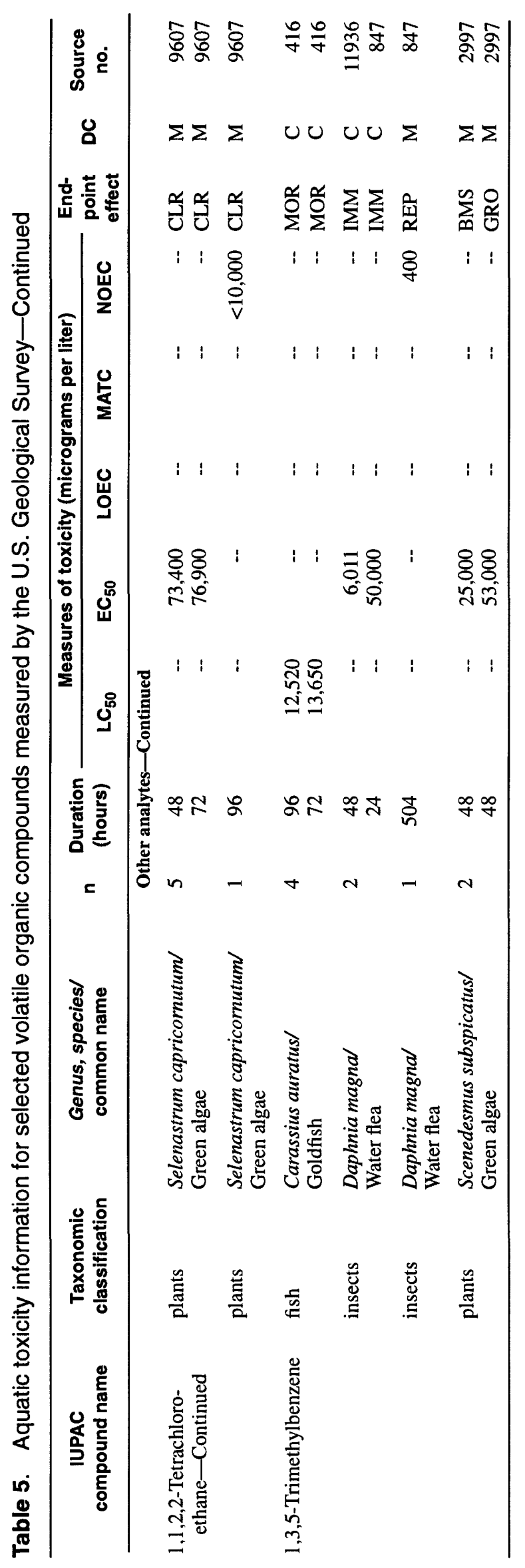




\section{REFERENCES CONTAINING AQUATIC TOXICITY INFORMATION}


Table 6. References containing aquatic toxicity information for selected volatile organic compounds as described in this study

[Source number may be used to retrieve citation from AQUIRE data base (U.S. Environmental Protection, 1996a)]

\footnotetext{
Source no.

(table 5)

References cited

46 Grover, P.B., Jr., 1985, A short-term behavioral bioassay for acute toxicity of water-borne pollutants [abs.]: Dissertation Abstracts International B--The Sciences and Engineering, v. 45, no. 12, pt. 1, p. 3671.

88 Figueroa, I. del C., and Simmons, M.S., 1991, Structure-activity relationships of chlorobenzenes using DNA measurement as a toxicity parameter in algae: Environmental Toxicology and Chemistry, v. 10. no. 3, p. 323-329.

Smith, A.D., Bharath, A., Mallard, C., Orr, D., Smith, K., Sutton, J.A., Vukmanich, J., McCarty, L.S., and Ozburn, G.W., 1991, The acute and chronic toxicity of ten chlorinated organic compounds to the American flagfish (Jordanella floridae): Archives of Environmental Contamination and Toxicology, v. 20, no. 1, p. 94-102.

212 Cowgill, U.M., and Milazzo, D.P., 1991, The sensitivity of Ceriodaphnia dubia and Daphnia magna to seven chemicals utilizing the three-brood test: Archives of Environmental Contamination and Toxico'ngy, v. 20, no. 2, p. 211-217.

257 Mayes, M.A., Shafer, T.J., and Barron, M.G., 1988, Critical evaluation of the fathead minnow 7- day static renewal test: Chemosphere, v. 17 , no. 11 , p. $2243-2252$.

416 Brenniman, G., Hartung, R., and Weber, W.J., Jr., 1976, A continuous flow bioassay method to evaluate the effect of outboard motor exhausts and selected aromatic toxicants on fish: Water Resources, v. 10, no. 2. p. 165-169.

418 Chandler, J.H., Jr., and Marking, L.L., 1979, Toxicity of fishery chemicals to the Asiatic clam, Corbicula manilensis: Progressive Fish-Culturist, v. 41, no. 3, p. 148-151.

448 Woodiwiss, F.S., and Fretwell, G., 1974, The toxicities of sewage effluents, industrial discharges and some chemical substances to brown trout (Salmo trutta) in the Trent River Authority Area: Water Pollution Ccntrol, v. 73, p. 396-405.

Moles, A., 1980, Sensitivity of parasitized coho salmon fry to crude oil, toluene, and naphthalene [Transactions]: American Fisheries Society, v. 109, no. 3, p. 293-297.

Thompson, R.S., and Carmichael, N.G., 1989, 1,1,1-Trichloroethane--Medium-term toxicity to carp, daphnids, and higher plants: Ecotoxicology and Environmental Safety, v. 17, no. 2, p. 172-182.

Paulet, G., and Vidal, M., 1975, Toxicity of some acrylic and methacrylic esters of acrylamide and pol "acrylamides: Archives des Maladies Professionnelles de Medecine du Travail et de Securite Sociale, v. 36, no. 1/2, p. 58-60.

508 Wallen, I.E., Greer, W.C., and Lasater, R., 1957, Toxicity to Gambusia affinis of certain pure chemic 71 in turbid waters: Sewage and Industrial Wastes, v. 29, no. 6, p. 695-711.

538 Birge, W.J., Black, J.A., Hudson, J.E., and Bruser, D.M., 1979, Embryo-larval toxicity tests with org^nic compounds, in Marking, L.L., and Kimerle, R.A., eds., Aquatic toxicology and hazard assessme 1 symposium, 2d: Philadelphia, American Society of Testing and Materials, ASTM STP 667, p. 131-147.

Leeuwangh, P., Bult, H., and Schneiders, L., 1975, Toxicity of hexachlorobutadiene in aquatic organ:sms, in Koneman, J.H., and Strik, J.J.T.W.A., eds., Sublethal effects of toxic chemicals on aquatic animals: Amsterdam, N.Y., Elsevier Science Publishers, p. 167-176.

542 Alabaster, J.S., 1969, Survival of fish in 164 herbicides, insecticides, fungicides, wetting agents and miscellaneous substances: International Pest Control, v. 11, no. 2, p. 29-35.

561 Lorz, H.W., Glenn, S.W., Williams, R.H., Kunkel, C.M., Norris, L.A., and Loper, B.R., 1979, Effect: of selected herbicides on smolting of coho salmon: Corvallis, Oreg., U.S. Environmental Protection Agency, Corvallis Environmental Research Laboratory Ecological Research Series, EPA-600/3-79-071, 102 p.

Birge, W.J., Black, J.A., and Bruser, D.M., 1979, Toxicity of organic chemicals to embryo-larval stages of fish: Washington, D.C., U.S. Environmental Protection Agency, Ecological Research Series, Office of Toxic Substances, EPA-560/11-79-007, 60 p. 
Table 6. References containing aquatic toxicity information for selected volatile organic compounds as described in this study-Continued

\begin{tabular}{cc}
$\begin{array}{c}\text { Source no. } \\
\text { (table 5) }\end{array}$ & References cited \\
\hline 632 & $\begin{array}{r}\text { Macek, K.J., Lindberg, M.A., Sauter, S., Buxton, K.S., and Costa, P.A., 1976, Toxicity of four pesticides to water } \\
\text { fleas and fathead minnows: Duluth, Minn., U.S. Environmental Protection Agency, Environmental Research } \\
\text { Laboratory, EPA-600/3-76-099, 68 p. }\end{array}$ \\
& $\begin{array}{c}\text { Johnson, W.W., and Finley, M.T., 1980, Handbook of acute toxicity of chemicals to fish and aquatic invertebrates: } \\
\text { Washington, D.C., U.S. Department of the Interior, Fish and Wildlife Service, Resource Publicatior 137, 98 p. }\end{array}$ \\
719 & $\begin{array}{c}\text { Mattson, V.R., Arthur, J.W., and Walbridge, C.T., 1976, Acute toxicity of selected organic compounds to fathead } \\
\text { minnows: Duluth, Minn., U.S. Environmental Protection Agency, Ecological Research Series, Env'ronmental } \\
\end{array}$ \\
& Research Laboratory, EPA-600/3-76-097, 12 p.
\end{tabular}

Pickering, Q.H., and Henderson, C., 1966, Acute toxicity of some important petrochemicals to fish: Jou nal of the Water Pollution Control Federation, v. 38, no. 9, p. 1419-1429.

Das, P.K.M.K., and Konar, S.K., 1988, Acute toxicity of petroleum products, crude oil and oil refinery effluent on plankton, benthic invertebrates and fish: Environmental Ecology, v. 6, no. 4, p. 885-891.

Kuhn, R., Pattard, M., Pernak, K., and Winter, A., 1989, Results of the harmful effects of selected water pollutants (anilines, phenols, aliphatic compounds) to Daphnia magna: Water Resources, v. 23, no. 4, p. 495-499.

Kuhn, R., Pattard, M., Pernak, K., and Winter, A., 1989, Results of the harmful effects of water pollutants to Daphnia magna in the 21-day reproduction test: Water Resources, v. 23, no. 4, p. 501-510.

Dawson, G.W., Jennings, A.L., Drozdowski, D., and Rider, E., 1977, The acute toxicity of 47 industrial cl $: m i c a l s ~ t o$ fresh and saltwater fishes: Journal of Hazardous Materials, v. 1, no. 4, p. 303-318.

Bond, C.E., Lewis, R.H., and Fryer, J.L., 1959, Toxicity of various herbicidal materials to fishes [Transaztions]: of the Second Seminar on Biological Problems in Water Pollution: Cincinnati, Ohio, Robert A. Taft Sanitation Engineering Center, Technical Report W60-3, p. 96-101.

Dowden, B.F., and Bennett, H.J., 1965, Toxicity of selected chemicals to certain animals: Journal of the Water Pollution Control Federation, v. 37, no. 9, p. 1308-1316.

Turnbull, H., Demann, J.G., and Weston, R.F., 1954, Toxicity of various refinery materials to fresh water fish: Industrial and Engineering Chemistry, v. 46, no. 2, p. 324-333.

Henderson, C., Pickering, Q.H., and Lemke, A.E., 1961, The effect of some organic cyanides (nitriles) on fish, in Industrial Waste Conference, 15th, Lafayette, Ind., 1961, Proceedings: Lafayette, Ind., Purdue University, ser. 106 , v. 65 , no. 2 , p. $120-130$.

Burdick, G.E., Dean, H.J., and Harris, E.J., 1964, Toxicity of aqualin to fingerling brown trout and bluegills: New York Fish and Game Journal, v. 11, no. 2, p. 106-114.

Patrick, R., Cairns, J., Jr., and Scheier, A., 1968, The relative sensitivity of diatoms, snails, and fish to twenty common constituents of industrial wastes: Progressive Fish-Culturist, v. 30, no. 3, p. 137-140.

Alexander, H.C., McCarty, W.M., and Bartlett, E.A., 1978, Toxicity of perchloroethylene, trichloroethylene, 1,1,1trichloroethane, and methylene chloride to fathead minnows: Bulletin of Environmental Contamination and Toxicology, v. 20 , no. 3 , p. 344-352.

Majewski, H.S., Klaverkamp, J.F., and Scott, D.P., 1978, Acute lethality, and sublethal effects of aceton?, ethanol, and propylene glycol on the cardiovascular and respiratory systems of rainbow trout (Salmo gairne $i$ ): Water Resources, v. 12, no. 4, p. 217-221.

Canton, J.H., and Adema, D.M.M., 1978, Reproducibility of short-term and reproduction toxicity experiments with Daphnia magna and comparison of the sensitivity of Daphnia magna with Daphnia pulex and Daphnia culullata in short-term experiments: Hydrobiologia, v. 59, no. 2, p. 135-140.

Louder, D.E., and McCoy, E.G., 1962, Preliminary investigations of the use of aqualin for collecting fish 9 , Annual Conference, 1962, Proceedings: Frankfort, Ky., Southeastern Association of Game and Fish Commissioners, v. 16 , p. $240-242$.

Bowman, M.C., Oller, W.L., Carins, T., Gosnell, A.B., and Oliver, K.H., 1981, Stressed bioassay systems for rapid screening of pesticide residues, pt. 1--Evaluation of bioassay systems: Archives of Environmental Contamination and Toxicology, v. 10, p. 9-24. 
Table 6. References containing aquatic toxicity information for selected volatile organic compounds as described in this study-Continued

\footnotetext{
Source no.

(table 5)

References cited

2193 Randall, T.L., and Knopp, P.V., 1980, Detoxification of specific organic substances by wet oxidation: Journal of the Water Pollution Control Federation, v. 52, no. 8, p. 2117-2130.

2215 Kauss, P.B., and Hutchinson, T.C., 1975, The effects of water-soluble petroleum components on the growth of Chlorella vulgaris Beijerinck: Environmental Pollution, v. 9, no. 3, p. 157-174.

2422 Van Hoogen, G., and Opperhuizen, A., 1988, Toxicokinetics of chlorobenzenes in fish: Environmen'al Toxicology and Chemistry, v. 7, no. 3, p. 213-219.

2470 Cardwell, R.D., Foreman, D.G., Payne, T.R., and Wilbur, D.J., 1978, Acute and chronic toxicity of four organic chemicals to fish: Duluth, Minn., U.S. Environmental Protection Agency, Environmental Research Laboratory, Contract 68-01-0711, 26 p. (unpublished data).

2644 Bentley, R.E., Heitmuller, T., Sleight, B.H., III, and Parrish, P.R., 1979, Acute toxicity of chloroform to bluegill (Lepomis macrochirus), rainbow trout (Salmo gairdneri), and pink shrimp (Penaeus duorarum): Washington, D.C., U.S. Environmental Protection Agency, Criteria Branch, WA-6-99-1414-B, 13 p.

Davis, J.T., and Hardcastle, W.S., 1959, Biological assay of herbicides for fish toxicity: Weeds, v. 7, p. 397-404.

Kuhn, R., and Pattard, M., 1990, Results of the harmful effects of water pollutants to green algae (Scenedesmus subspicatus) in the cell multiplication inhibition test: Water Resources, v. 24, no. 1, p. 31-38.

3029 Rajini, P.S., Krishnakumari, M.K., and Majumder, S.K., 1989, Cytotoxicity of certain organic solverts and organophosphorus insecticides to the ciliated protozoan Paramecium caudatum: Microbios, v. 59, p. 157-163.

3217 Geiger, D.L., Brooke, L.T., and Call, D.J., 1990, Acute toxicities of organic chemicals to fathead minnows (Pimephales promelas), v. 5: Superior, University of Wisconsin, Center for Lake Superior Environmental Studies, $332 \mathrm{p}$.

3279 Van Leeuwen, C.J., Adema, D.M.M., and Hermens, J., 1990, Quantitative structure-activity relationships for fish early life stage toxicity: Aquatic Toxicology, v. 16, no. 4, p. 321-334.

3283 Smith, S.B., Savino, J.F., and Blouin, M.A., 1988, Acute toxicity to Daphnia pulex of six classes of chemical compounds potentially hazardous to Great Lakes aquatic biota: Journal of Great Lakes Research, v. 14, no. 4, p. 394-404.
}

3350 Vykusova, B., and Svobodova, Z., 1989, Evaluation of the toxicity of the cyadox growth stimulator to aquatic organisms: Biological and Chemical Factors in Animal Production -- Veterinary Science, v. 25, no. 1, p. 41-49. [Abstract in English.]

3550 Herman, D.C., Inniss, W.E., and Mayfield, C.I., 1990, Impact of volatile aromatic hydrocarbons, alone and in combination, on growth of the freshwater alga Selenastrum capricornutum: Aquatic Toxicology, v. 18, no. 2 , p. $87-100$.

3690 Call, D.J., Brooke, L.T., Ahmad, N., and Vaishnav, D.D., 1981, Aquatic pollutant hazard assessmens and development of a hazard prediction technology by quantitative structure-activity relationships: Superior, University of Wisconsin, Center for Lake Superior Environmental Studies, 2d Quarterly Report. U.S. Environmental Protection Agency Cooperative Agreement No. CR 809234-01-0, 74 p.

Kimball, G., 1978, The effects of lesser known metals and one organic to fathead minnows (Pimephales promelas and Daphnia magna): Minneapolis, University of Minnesota, Department of Entomology, Fisharies and Wildlife, $88 \mathrm{p}$.

3881 Merlin, G., Thiebaud, H., Blake, G., Sembiring, S., and Alary, J., 1992, Mesocosms' and microcosms' utilization for the ecotoxicity evaluation of dichloromethane, a chlorinated solvent: Chemosphere, v. 24, no. 1, p. 37-50.

3910 Marchini, S., Tosato, M.L., Norberg-King, T.J., Hammermeister, D.E., and Hoglund, M.D., 1992, Lethal and sublethal toxicity of benzene derivatives to the fathead minnow, using a short-term test: Envircnmental Toxicology and Chemistry, v. 11, no. 2, p. 187-195.

Ghazaly, K.S., 1991, Physiological alterations in Claria lazera induced by two different pollutants: Water, Air, and Soil Pollution, v. 60, no. 1/2, p. 181-187.

4038

Benoit-Guyod, J.L., Andre, C., and Clavel, K., 1984, Chlorophenols--Degradation and toxicity: Journal of French Hydrology, v. 15, no. 3, p. 249-266. [Abstract in English.] 
Table 6. References containing aquatic toxicity information for selected volatile organic compounds as described in this study-Continued

\begin{tabular}{cc}
$\begin{array}{c}\text { Source no. } \\
\text { (table 5) }\end{array}$ & References cited \\
\hline
\end{tabular}

4072 Roghair, C.J., Buijze, A., Yedema, E.S.E., and Hermens, J.L.M., 1994, A QSAR for base-line toxicity to the midge Chironomus riparius: Chemosphere, v. 28, no. 5, p. 989-997.

4154 Call, D.J., Brooke, L.T., and Ahmad, N., 1981, Estimates of "no effect" concentrations of selected pestic: des in freshwater organisms--3d quarterly progress report: Superior, University of Wisconsin, U.S. Environmental Protection Agency, Cooperative Agreement No. CR 806864030, 84 p.

4189 Masten, L.W., Boeri, R.L., and Walker, J.D., 1994, Strategies employed to determine the acute aquatic tc xicity of ethyl benzene, a highly volatile, poorly water-soluble chemical: Ecotoxicological and Environmental Safety, v. 27 , no. 3 , p. $335-348$.

4399 Darville, R.G., 1982, The effects of naphthalene on the physiology and life cycle of Chironomus attenuatus and Tanytarsus dissimilis: Stillwater, Oklahoma State University, Ph.D. dissertation, 85 p.

4433 Ahmad, N., Benoit, D., Brooke, L., Call, D., Carlson, A., DeFoe, D., Huot, J., Moriarity, A., Richter, J., Shubat, P., Veith, G., and Wallbridge, C., 1984, Aquatic toxicity tests to characterize the hazard of volatile organic chemicals in water--A toxicity data summary--pts. 1 and 2: Duluth, Minn., U.S. Environmental Prctection Agency, Environmental Research Laboratory, EPA 600/3-84-009, 103 p.

5087 Pearson, J.G., Glennon, J.P., Barkley, J.J., and Highfill, J.W., 1979, An approach to the toxicological eval iation of a complex industrial wastewater, in Marking, L.L., and Kimerle, R.A., eds., Aquatic toxicology and $\mathrm{l}$ azard assessment, 2d symposium: Philadelphia, American Society of Testing and Materials, ASTM STP 667, p. 284-301.

5184 LeBlanc, G.A., 1980, Acute toxicity of priority pollutants to water flea (Daphnia magna): Bulletin of Environmental Contaminant Toxicology, v. 24, no. 5, p. 684-691.

Anderson, D.R., and Lusty, E.B., 1980, Acute toxicity and bioaccumulation of chloroform to four species of freshwater fish--Salmo gairdneri, rainbow trout, Lepomis macrochirus, bluegill, Micropterus salmoides, largemouth bass, Ictalurus punctatus, channel catfish: Washington, D.C., U.S. Nuclear Regulation Committee, Report No. CR-0893, 33 p.

5272 Black, J.A., and Birge, W.J., 1980, An avoidance response bioassay for aquatic pollutants: Lexington, Uriversity of Kentucky, Water Resource Research Institute, Research Report No. 123, 34 p.

5331 Canton, J.H., Wegman, R.C.C., Mathijssen-Spiekman, E.A.M., and Wammes, J.Y., 1980, Hydrobiologic 71 toxicological research with methylbromide: National Institute of Public Health and Environmental Hygiene, Report No. 105, 804 p. [In Dutch.]

5580 Stoss, F.W., and Haines, T.A., 1979, The effects of toluene on embryos and fry of the Japanese Medaka, Oryzias latipes, with a proposal for rapid determination of maximum acceptable toxicant concentration: Environmental Pollution, v. 20, no. 2, p. 139-148.

5590 Buccafusco, R.J., Ells, S.J., and LeBlanc, G.A., 1981, Acute toxicity of priority pollutants to bluegill (Lepomis macrochirus): Bulletin of Environmental Contamination and Toxicology, v. 26, no. 4, p. 446-452.

5622 Moles, A., Rice, S.D., and Korn, S., 1979, Sensitivity of Alaskan freshwater and anadromous fishes to Prudhoe Bay crude oil and benzene [Transactions]: American Fisheries Society, v. 108, no. 4, p. 408-414.

5675 Hermens, J., Canton, H., Steyger, N., and Wegman, R., 1984, Joint effects of a mixture of 14 chemicals on mortality and inhibition of reproduction of Daphnia magna: Aquatic Toxicology, v. 5, no. 4, p. 315-322.

5700 Berry, W.O., and Brammer, J.D., 1977, Toxicity of water-soluble gasoline fractions to fourth-instar larvae of the mosquito, Aedes aegypti L.: Environmental Pollution, v. 13, no. 3, p. 229-234.

5718 Bringmann, G., and Kuhn, R., 1977, Results of the damaging effect of water pollutants on Daphnia magna: Zietschrift fuer Wasser- und Abwasser- Forschung, v. 10, no. 5, p. 161-166. [In German with English abstract.]

5735 Curtis, M.W., Copeland, T.L., and Ward, C.H., 1978, Aquatic toxicity of substances proposed for spill Frevention regulation, in National Conference on Control of Hazardous Material Spills, Miami Beach, Fla., April 11-13, 1989, Proceedings: Rockville, Md., Oil Spill Control Association of America, p. 93-103.

Dill, D.C., McCarty, W.M., Alexander, H.C., and Bartlett, E.A., 1980, Toxicity of 1,1-dichloroethylene (vinylidene chloride) to aquatic organisms: Duluth, Minn., U.S. Environmental Protection Agency, Environmental Research Laboratory, Ecological Research Series, EPA-600/3-80-057, 17 p. 
Table 6. References containing aquatic toxicity information for selected volatile organic compounds as described in this study-Continued

\author{
Source no. \\ (table 5) \\ References cited
}

5773 Jensen, R.A., 1978, A simplified bioassay using finfish for estimating potential spill damage, in Naticnal Conference on Control of Hazardous Material Spills, Miami Beach, Fla., April 11-13, 1989, Proreedings: Rockville, Md., Oil Spill Control Association of America, p. 104-108.

5876 Bottger, A., 1988, Belastung der Anwohner von Chemisch-Reinigungsanlegen durch Tetrachlorethylen, Votrag: Tagung der Deutschen Gesellschaft fr Hygiene und Mikrobiologie, Kiel, v. 9, p. 29-30. [In German.]

5938 Slooff, W., 1979, Detection limits of a biological monitoring system based on fish respiration: Bulletin of Environmental Contamination and Toxicology, v. 23, no. 4-5, p. 517-523.

6002 Ferrando, M.D., and Andreu-Moliner, E., 1992, Acute toxicity of toluene, hexane, xylene, and benzene to the rotifers Brachionus calyciflorus and Brachionus plicatilis: Bulletin of Environmental Contamination and Toxicology, v. 49, no. 2, p. 266-271.

Munoz, M.J., and Tarazona, J.V., 1993, Synergistic effect of two- and four-component combinations of the polycyclic aromatic hydrocarbons--Phenanthrene, anthracene, naphthalene and acenaphthene or Daphnia magna: Bulletin of Environmental Contamination and Toxicology, v. 50, no. 3, p. 363-368.

Wester, P.W., Canton, J.H., and Dormans, J.A.M.A., 1988, Pathological effects in freshwater fish Poecilia reticulata (guppy) and Oryzias latipes (medaka) following methyl bromide and sodium bromide exposure: Aquatic Toxicology, v. 12, no. 4, p. 323-344.

Birge, W.J., Black, J.A., and Kuehne, R.A., 1980, Effects of organic compounds on amphibian reproduction: Lexington, University of Kentucky, Water Resource Research Institute, Research Report No. 121, 39 p.

Trabalka, J.R., and Burch, M.B., 1978, Investigation of the effects of halogenated organic compouncs produced in cooling systems and process effluents on aquatic organisms, in Jolley, R.L., Gorchev, H., and Hamilton, D.R., Jr., eds., Water chlorination--Environmental impact and health effects: p. 163-173.

Mattice, J.S., Tsai, S.C., Burch, M.B., and Beauchamp, J.J., 1981, Toxicity of trihalomethanes to com mon carp embryos [Transactions]: American Fisheries Society, v. 110, no. 2, p. 261-269.

Geiger, J.G., Buikema, A.L., Jr., and Cairns, J., Jr., 1980, A tentative seven-day test for predicting effects of stress on populations of Daphnia pulex, in Eaton, J.G., Parrish, P.R., and Hendricks, A.C., eds., Aquatic toxicology and hazard assessment symposium, 3d: Philadelphia, American Society of Testing and Material $\varsigma$ ASTM STP 707, p. 13-26.

6516 Janssen, C.R., and Persoone, G., 1993, Rapid toxicity screening tests for aquatic biota, 1--Methodolo :yy and experiments with Daphnia magna: Environmental Toxicology and Chemistry, v. 12, p. 711-717.

Hodson, P.V., Parisella, R., Blunt, B., Gray, B., Kaiser, K.L.E., 1991, Quantitative structure-activity re'ationships for chronic toxicity of phenol, $p$-chlorophenol, 2,4-dichlorophenol, pentachlorophenol, $p$-nitrophenol, and 1,2,4trichlorobenzene to early life stages of rainbow trout (Oncorhynchus mykiss): Canadian Technical Report of Fisheries and Aquatic Sciences, v. 1784, 55 p.

6984 Vigano, L., 1993, Reproductive strategy of Daphnia magna and toxicity of organic compounds: Wat $\times r$ Resources, v. 27 , no. 5 , p. 903-909.

7049 Darville, R.G. and Wilhm, J.L., 1984, The effect of naphthalene on oxygen consumption and hemoglsbin concentration in Chironomus attenuatus and on oxygen consumption and life cycle of tanytarsus dissimilis: Environmental Toxicology and Chemistry, v. 3, no. 1, p. 135-141.

Sijm, D.T.H.M., Schipper, M., and Opperhuizen, A., 1993, Toxicokinetics of halogenated benzenes in fish--Lethal body burden as a toxicological end point: Environmental Toxicology and Chemistry, v. 12, p. 1117-1127.

7367 Yarzhombek, A.A., Mikulin, A.E., and Zhdanova, A.N., 1991, Toxicity of some substances to fish in relation to form of exposure: Journal of Ichthyology, v. 31, no. 7, p. 99-106.

7398 Bailey, H.C., Liu, D.H.W., and Javitz, H.A., 1985, Time/toxicity relationships in short-term static, dynamic, and plug-flow bioassays, in Bahner, R.C., and Hansen, D.J., eds., Aquatic toxicology and hazard ass :ssment symposium, 8th: Philadelphia, American Society for Testing and Materials, ASTM STP 891, p. 193-212. 
Table 6. References containing aquatic toxicity information for selected volatile organic compounds as described in this study-Continued

\author{
Source no. \\ (table 5) \\ References cited
}

7884 Ziegenfuss, P.S., Renaudette, W.J., and Adams, W.J., 1986, Methodology for assessing the acute toxicity of chemicals sorbed to sediments--Testing the equilibrium partitioning theory, in Poston, T.M., and Pu-dy, R., eds., Aquatic toxicology and environmental fate symposium, 9th: Philadelphia, American Society for Testing and Materials, ASTM STP 921 v. 9, p. 479-493.

8099 Keller, A.E., 1993, Acute toxicity of several pesticides, organic compounds, and a wastewater effluent to the freshwater mussel, Anodonta imbecilis, Ceriodaphnia dubia, and Pimephales promelas: Bulletin of Environmental Contamination and Toxicology, v. 51, no. 5, p. 696-702.

9158 Nishiuchi, Y., and Yoshida, K., 1972, Toxicities of pesticides to some fresh water snails: Bulletin of the Agricultural Chemicals Inspection Station, v. 12, p. 86-92.

9196 Bazin, C., Chambon, P., Bonnefille, M., and Larbaigt, G., 1987, Compared sensitivity of luminescent marine bacteria (Photobacterium phosphoreum) and Daphnia bioassays: Sciences de l'Leau, v. 6, p. 403-413.

Snell, T.W., Moffat, B.D., Janssen, C., and Persoone, G., 1991, Acute toxicity tests using rotifers, 4-- Effects of cyst age, temperature, and salinity on the sensitivity of Barachionus calyciflorus: Ecotoxicology and Environmental Safety, v. 21, no. 3, p. 308-317.

U.S. Environmental Protection Agency, 1978, In-depth studies on health and environmental impacts of s?lected water pollutants: Duluth, Minn., U.S. Environmental Progection Agency, Contract No. 68-01-4646. 9 p.

Slooff, W., and Baerselman, R., 1980, Comparison of the usefulness of the Mexican Axolotl (Ambystoma mexicanum) and the clawed toad (Xenopus laevis) in toxicological bioassays: Bulletin of Environmental Contamination and Toxicology, v. 24, no. 3, p. 439-443.

Black, J.A., Birge, W.J., Westerman, A.G., and Francis, P.C., 1983, Comparative aquatic toxicology of aromatic hydrocarbons: Fundamental and Applied Toxicology, v. 3, no. 9/10, p. 353-358.

Tonogai, Y., Ogawa, S., Ito, Y., and Iwaida, M., 1982, Actual survey on MTL (Median Tolerance Limit) values of environmental pollutants, especially on amines, nitriles, aromatic nitrogen compounds and artificial dyes: Journal of Toxicological Sciences, v. 7, no. 3, p. 193-203.

Veith, G.D., Call, D. J., and Brooke, L.T., 1983, Estimating the acute toxicity of narcotic industrial chemicals to fathead minnows, in Bishop, W.E., Cardwell, R.D., and Heidolph, B.B., eds., Aquatic toxicology and hazard assessment symposium, 6th: Philadelphia, American Society for Testing and Materials, ASTM STP 802, p. 90-97.

10359 Crider, J.Y., Wilhm, J., and Harmon, H.J., 1982, Effects of naphthalene on the hemoglobin concentration and oxygen uptake of Daphnia magna: Bulletin of Environmental Contamination and Toxicology, v. 28, p. 52-57.

10366

Heitmuller, P.T., Hollister, T.A., and Parrish, P.R., 1981, Acute toxicity of 54 industrial chemicals to sheepshead minnows (Cyprinodon variegatus): Bulletin of Contamination and Toxicology, v. 27, no. 5, p. 596-604.

10432 Mayes, M.A., Alexander, H.C., and Dill, D.C., 1983, A study to assess the influence of age on the response of fathead minnows in static acute toxicity tests: Bulletin of Contamination and Toxicology, v. 31, no. 2, p. 139-147.

Segers, J.H.L., Temmink, J.H.M., Van den Berg, J.H.J., and Wegman, R.C.C., 1984, Morphological chan ${ }^{2}$ es in the gill of carp (Cyprinus carpio L.) exposed to acutely toxic concentrations of methyl bromide: Water P.esources, v. 18 , no. 11 , p. $1437-1441$.

10579 Call, D.J., Brooke, L.T., Ahmad, N., and Richter, J.E., 1983, Toxicity and metabolism studies with EPA priority pollutants and related chemicals in freshwater organisms: Duluth, Minn., U.S. Environmental Protection Agency, EPA 600/3-83-095, 120 p.

LeBlanc, G.A., and Surprenant, D.C., 1983, The acute and chronic toxicity of acetone, dimethyl formanide, and triethylene glycol to Daphnia magna (Straus): Archives of Environmental Contamination and Toxirology, v. 12 , no. 3 , p. $305-310$. 
Table 6. References containing aquatic toxicity information for selected volatile organic compounds as described in this study-Continued

\begin{tabular}{ll}
$\begin{array}{c}\text { Source no. } \\
\text { (table 5) }\end{array}$ References cited \\
\hline
\end{tabular}

10712 Calamari, D., Galassi, S., and Setti, F., 1982, Evaluating the hazard of organic substances on aquatic life--The paradichlorobenzene example: Ecotoxicology and Environmental Safety, v. 6, no. 4, p. 369-378.

10775 Phipps, G.L., and Holcombe, G.W., 1985, A method for aquatic multiple species toxicant testing--Acute toxicity of 10 chemicals to 5 vertebrates and 2 invertebrates: Environmental Pollution Series A--Ecological and Biological, v. 38, no. 2, p. 141-157.

10805 Bobra, A., Shiu, W.Y., and MacKay, D., 1985, Quantitative structure-activity relationships for the ac'ite toxicity of chlorobenzenes to Daphnia magna: Environmental Toxicology and Chemistry, v. 4, no. 3, p. 297-305.

10810 Cowgill, U.M., Takahashi, I.T., and Applegath, S.L., 1985, A comparison of the effect of four benchmark chemicals on Daphnia magna and Ceriodaphnia dubia affinis tested at two different temperatures: Envircnmental Toxicology and Chemistry, v. 4, no. 3, p. 415-422.

10954 Holcombe, G.W., Phipps, G.L., Knuth, M.L., and Felhaber, T., 1984, The acute toxicity of selected s`lbstituted phenols, benzenes and benzoic acid esters to fathead minnows, Pimephales promelas: Environmental Pollution Series A--Ecological and Biological, v. 35, no. 4, p. 367-381.

11181 Mount, D.I., and Norberg, T.J., 1984, A seven-day life-cycle cladoceran toxicity test: Environmental Toxicology and Chemistry, v. 3, no. 3, p. 425-434.

11227 Walbridge, C.T., Fiandt, J. T., Phipps, G.L., and Holcombe, G.W., 1983, Acute toxicity of ten chlorinated aliphatic hydrocarbons to the fathead minnow (Pimephales promelas): Archives of Environmental Contamination and Toxicology, v. 12, no. 6, p. 661-666.

11282 Saha, M.K., and Konar, S.K., 1983, Acute toxicity of some petroleum pollutants to plankton and fist: Environmental Ecology, v. 1, no. 1, p. 117-119.

11455 Van Leeuwen, C.J., Maas-Diepeveen, J.L., Niebeek, G., Vergouw, W.H.A., Griffioen, P.S., and Luijken, M.W., 1985, Aquatic toxicological aspects of dithiocarbamates and related compounds, 1--Short-term toxicity tests: Aquatic Toxicology, v. 7, no. 3, p. 145-164.

11677 Geyer, H., Scheunert, I., and Korte, F., 1985, The effects of organic environmental chemicals on the growth of the alga Scenedesmus subspicatus--A contribution to environmental biology: Chemosphere, v. 14, no. 9, p. 13551369.

11725 Millemann, R.E., Birge, W.J., Black, J.A., Cushman, R.M., Daniels, K.L., Franco, P.J., Giddings, J.M1., McCarthy, J.F., and Stewart, A.J., 1984, Comparative acute toxicity to aquatic organisms of components of coal-derived synthetic fuels [Transactions]: American Fisheries Society, v. 113, no. 1, p. 74-85.

11926 Abernethy, S., Bobra, A.M., Shiu, W.Y., Wells, P.G., and MacKay, D., 1986, Acute lethal toxicity of hydrocarbons and chlorinated hydrocarbons to two planktonic crustaceans--The key role of organism-water partitioning: Aquatic Toxicology, v. 8, no. 3, p. 163-174.

11936 Bobra, A.M., Shiu, W.Y., and MacKay, D., 1983, A predictive correlation for the acute toxicity of hydrocarbons and chlorinated hydrocarbons to the water flea (Daphnia magna): Chemosphere, v. 12, no. 9-1C, p. 1121-1129.

11951 Ewell, W.S., Gorsuch, J.W., Kringle, R.O., Robillard, K.A., and Spiegel, R.C., 1986, Simultaneous evaluation of the acute effects of chemicals on seven aquatic species: Environmental Toxicology and Chemistry, v. 5. no. 9 , p. 831-840.

12004 Thurston, R.V., Gilfoil, T.A., Meyn, E.L., Zajdel, R.K., Aoki, T.L., and Veith, G.D., 1985, Comparat've toxicity of ten organic chemicals to ten common aquatic species: Water Resources, v. 19, no. 9, p. 1145-1155.

Carlson, A.R., 1987, Effects of lowered dissolved oxygen concentration on the toxicity of 1,2,4-trichlorobenzene to fathead minnows: Bulletin of Environmental Contamination and Toxicology, v. 38, p. 667-673.

Carlson, A.R., and Kosian, P.A., 1987, Toxicity of chlorinated benzenes to fathead minnows (Pimephales promelas): Archives of Environmental Contamination and Toxicology, v. 16, no. 2, p. 129-135.

12152

De Zwart, D., and Slooff, W., 1987, Toxicity of mixtures of heavy metals and petrochemicals to Xen opus laevis: Bulletin of Environmental Contamination and Toxicology, v. 38, no. 2, p. 345-351. 
Table 6. References containing aquatic toxicity information for selected volatile organic compounds as described in this study-Continued

\section{Source no. \\ (table 5) \\ References cited}

12182 McKim, J.M., Schmieder, P.K., Niemi, G.J., Carlson, R.W., and Henry, T.R., 1987, Use of respiratorycardiovascular responses of rainbow trout (Salmo gairdneri) in identifying acute toxicity syndromes in fish, 2--Malathion, carbaryl, acrolein and benzaldehyde: Environmental Toxicology and Chemistry, v. 6, p. 313328.

12258 Elnabarawy, M.T., Welter, A.N., and Robideau, R.R., 1986, Relative sensitivity of three daphnid species to selected organic and inorganic chemicals: Environmental Toxicology and Chemistry, v. 5, no. 4, p. 393-398.

12405 Devlin, E.W., 1983, Developmental studies on the fathead minnow (Pimephales promelas Raf.), 1--The prehatching development of the fathead minnow, 2--The acute effects of toluene on three age groups of fathead minnows, 3--The effect of toluene on the prehatching develpment of the fathead minnow [abs.]: Dissertation Abstracts International B--The Sciences and Engineering, v. 43, no. 11, pt. 2, p. 3502.

12447 Geiger, D.L., Northcott, C.E., Call, D.J., and Brooke, L.T., 1985, Acute toxicities of organic chemicals to fathead minnows (Pimephales promelas), v. 2: Superior, University of Wisconsin, Center for Lake Superior Environmental Studies, $326 \mathrm{p}$.

12448 Brooke, L.T., Call, D.J., Geiger, D.L., and Northcott, C.E., 1984, Acute toxicities of organic chemicals to fathead minnows (Pimephales promelas), v. 1: Superior, University of Wisconsin, Center for Lake Superior Environmental Studies, $414 \mathrm{p}$.

12497 Tsuji, S., Tonogai, Y., Ito, Y., and Kanoh, S., 1986, The influence of rearing temperatures on the toxicity cf various environmental pollutants for killifish (Oryzias latipes): Journal of Hygenic Chemistry, v. 32, no. 1, p. 46-53.

12513 Yoshioka, Y., Ose, Y., and Sato, T., 1986, Correlation of the five test methods to assess chemical toxicity and relation to physical properties: Ecotoxicology and Environmental Safety, v. 12, no. 1. p. 15-21.

12597 Stratton, G.W., 1987, Toxic effects of organic solvents on the growth of blue-green algae: Bulletin of Environmental Contamination and Toxicology, v. 38, no. 6, p. 1012-1019.

12665 Holcombe, G.W., Phipps, G.L., Sulaiman, A.H., and Hoffman, A.D., 1987, Simultaneous multiple specie testing-Acute toxicity of 13 chemicals to 12 diverse freshwater amphibian, fish, and invertebrate families: Archives of Environmental Contamination and Toxicology, v. 16, p. 697-710.

12858 Geiger, D.L., Poirier, S.H., Brooke, L.T., and Call, D.J., 1986, Acute toxicities of organic chemicals to fathead minnows (Pimephales promelas), v. 3: Superior, University of Wisconsin, Center for Lake Superior Environmental Studies, $328 \mathrm{p}$.

12859 Geiger, D.L., Call, D.J., and Brooke., L.T., 1988, Acute toxicities of organic chemicals to fathead minnov's (Pimephales promelas), v. 4: Superior, University of Wisconsin, Center for Lake Superior Environmental Studies, $355 \mathrm{p}$.

12872 Deneer, J.W., Seinen, W., and Hermens, J.L.M., 1988, Growth of Daphnia magna exposed to mixtures of chemicals with diverse modes of action: Ecotoxicology and Environmental Safety, v. 15, no. 1, p. 72-77.

13142 Galassi, S., Mingazzini, M., Vigano, L., Cesareo, D., and Tosato, M.L., 1988, Approaches to modeling toxic responses of aquatic organisms to aromatic hydrocarbons: Ecotoxicology and Environmental Safety, v. 16, no. 2, p. 158-169.

Erben, R., and Pisl, Z., 1993, Acute toxicity for some evaporating aromatic hydrocarbons for freshwater snails and crustaceans: Internationale Revueder Gesamten Hydrobiologie, v. 78, no. 1, p. 161-167.

Van der Zandt, P.T.J., Heinis, F., and Kikkert, A., 1994, Effects of narcotic industrial pollutants on behaviour of midge larvae (Chironomus riparius (Meigen), Diptera)--A quantitative structure-activity relationship: Aquatic Toxicology, v. 28, no. 3/4, p. 209-221.

15131 Degraeve, G.M., Elder, R.G., Woods, D.C., and Bergman, H.L., 1982, Effects of naphthalene and benzen? on fathead minnows and rainbow trout: Archives of Environmental Contamination and Toxicology, v. 11, p. $87-490$.

15149 Adema, D.M.M.M., and Vink, G.J., 1981, A comparative study of the toxicity of 1,1,2-trichloroethane, d eldrin, pentachlorophenol, and 3,4-dichloroaniline for marine and freshwater organisms: Chemosphere, v. 10, no. 6, p. 533-544. 
Table 6. References containing aquatic toxicity information for selected volatile organic compound as described in this study-Continued

\begin{tabular}{|c|c|}
\hline $\begin{array}{l}\text { Source no. } \\
\text { (table 5) }\end{array}$ & References cited \\
\hline 15191 & $\begin{array}{l}\text { Moles, A., Bates, S., Rice, S.D., and Korn, S., 1981, Reduced growth of coho salmon fry exposed to two petroleum } \\
\text { components, toluene and naphthalene, in fresh water [Transactions]: American Fisheries Society, v. 110, no. 3, } \\
\text { p. 430-436. }\end{array}$ \\
\hline 15192 & $\begin{array}{l}\text { Nishiuchi, Y., and Hashimoto, Y., 1967, Toxicity of pesticide ingredients to some fresh water organisms: Science } \\
\text { Pest Control/Botyu-Kagaku, v. 32, no. 1, p. 5-11. [In Japanese with English abstract.] }\end{array}$ \\
\hline 15211 & $\begin{array}{l}\text { Maynard, D.J., and Weber, D.D., 1981, Avoidance reactions of juvenile coho salmon (Oncorhynchus kisutch) to } \\
\text { monocyclic aromatics: Canadian Journal of Fisheries and Aquatic Sciences, v. 38, no. 7, p. 772-778. }\end{array}$ \\
\hline 15293 & $\begin{array}{l}\text { Geiger, J.G., and Buikema, A.L., Jr., 1982., Hydrocarbons depress growth and reproduction of Daphnia pulex } \\
\text { (Cladocera): Canadian Journal of Fisheries and Aquatic Sciences, v. 3, no. 6, p. 830-836. }\end{array}$ \\
\hline 15301 & $\begin{array}{l}\text { Benoit, D.A., Puglisi, F.A., and Olson, D.L., 1982., A fathead minnow Pimephales promelas early life stage } \\
\text { toxicity test method evaluation and exposure to four organic chemicals: Environmental Pollution, Series A-- } \\
\text { Ecological and Biological, v. 28, no. 3, p. 189-197. }\end{array}$ \\
\hline 15337 & $\begin{array}{l}\text { Trucco, R.G., Engelhardt, F.R., and Stacey, B., 1983, Toxicity, accumulation and clearance of aromatic } \\
\text { hydrocarbons in Daphnia pulex: Environmental Pollution, Series A--Ecological and Biological, v. } 31 \text {, no. } 3 . \\
\text { p. 191-202. }\end{array}$ \\
\hline 15418 & $\begin{array}{l}\text { Black, J.A., Birge, W.J., McDonnell, W.E., Westerman, A.G., Ramey, B.A., and Bruser, D.M., 1982, The aquatic } \\
\text { toxicity of organic compounds to embryo-larval stages of fish and amphibians: Lexington, Un iversity of } \\
\text { Kentucky, Water Resources Research Institute, Research Report No. 133, } 61 \text { p. }\end{array}$ \\
\hline 15486 & $\begin{array}{l}\text { Correa, M., and Coler, R., 1983, Enhanced oxygen uptake rates in dragonfly nymphs (Somatochlorc cingulata) as } \\
\text { an indication of stress from naphthalene: Bulletin of Environmental Contamination and Toxicology, v. } 30 \text {, } \\
\text { no. } 3 \text {, p. 269-276. }\end{array}$ \\
\hline 15526 & $\begin{array}{l}\text { Calamari, D., Galassi, S., Setti, F., and Vighi., M., 1983, Toxicity of selected chlorobenzenes to aquatic organisms: } \\
\text { Chemosphere, v. } 12 \text {, no. } 2 \text {, p. } 253-262 \text {. }\end{array}$ \\
\hline 15788 & $\begin{array}{l}\text { Slooff, W., 1983, Benthic macroinvertebrates and water quality assessment--Some toxicological cor siderations: } \\
\text { Aquatic Toxicology, v. 4, p. 73-82. }\end{array}$ \\
\hline 15923 & $\begin{array}{l}\text { Qureshi, A.A., Flood, K.W., Thompson, S.R., Janhurst, S.M., Inniss, C.S., and Rokosh, D.A., 1982, Comparison of } \\
\text { a luminescent bacterial test with other bioassays for determining toxicity of pure compounds and complex } \\
\text { effluents, in Pearson, J.G., Foster, R.B., and Bishop, W.E., eds., Aquatic toxicology and hazard assessments } \\
\text { symposium, 5th: Philadelphia, American Society for Testing and Materials, ASTM STP 766, p. 79-196. }\end{array}$ \\
\hline 15981 & $\begin{array}{l}\text { Richter, J.E., Peterson, S.F., and Kleiner, C.F., 1983, Acute and chronic toxicity of some chlorinated benzenes, } \\
\text { chlorinated ethanes, and tetrachloroethylene to Daphnia magna: Archives of Environmental Contamination } \\
\text { and Toxicology, v. } 12 \text {, no. 6, p. } 679-684 .\end{array}$ \\
\hline
\end{tabular}

